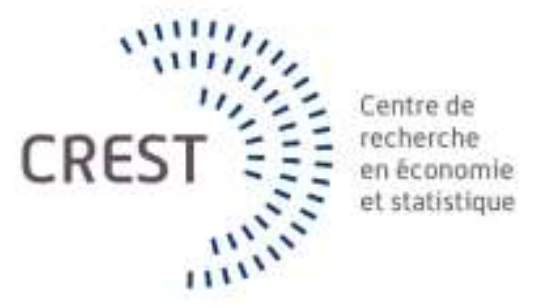

Série des Documents de Travail

\title{
$n^{\circ}$ 2016-31 \\ Stationary Bubble Equilibria in Rational Expectation Models \\ C.Gouriéroux ${ }^{1}$ \\ J.Jasiak ${ }^{2}$ \\ A.Monfort ${ }^{3}$
}

Les documents de travail ne reflètent pas la position du CREST et n'engagent que leurs auteurs.

Working papers do not reflect the position of CREST but only the views of the authors.

\footnotetext{
${ }^{1}$ CREST and University of Toronto. E-mail: christian.gouriéroux@ensae.fr

${ }^{2}$ York University, Canada. E-mail: jasiakj@yorku.ca

${ }^{3}$ CREST. E-mail: alain.monfort@ensae.fr
} 


\title{
STATIONARY BUBBLE EQUILIBRIA IN RATIONAL EXPECTATION MODELS
}

\author{
C. Gourieroux ${ }^{(1)}$, J. Jasiak ${ }^{(2)}$ and A. Monfort ${ }^{(3)}$
}

October, 23, 2016

The first author gratefully acknowledges financial support of the chair ACPR/Risk Foundation : Regulation and Systemic Risks. We thank S., Auray, I. Ekeland for helpful discussions on the transversality conditions.

\footnotetext{
${ }^{1}$ CREST and University of Toronto.

${ }^{2}$ York University, Canada

${ }^{3}$ CREST.
} 


\section{Stationary Bubble Equilibria in Rational Expectation Models}

Abstract

A linear rational expectation model with current expectations can admit a unique linear stationary dynamic equilibrium for a set of specific parameter values. This paper shows that a multiplicity of stationary dynamic equilibria may arise due to the existence of nonlinear stationary equilibria. These nonlinear equilibria can display bubbles and/or volatility induced mean reversion, consistently with the self-fulfilling prophecies that characterize the rational expectation equilibria. The stationary nonlinear dynamic equilibria

require a revised approach in the identification issue, in the impulse response analysis in rational expectation models, or in the test of the present value model that are also discussed in this paper.

Keywords : Rational Expectation, Equilibrium, Stationary Martingale, Speculative Bubble, Volatility Induced Mean-Reversion, Stochastic Economy, Transversality Condition, Identification, Present Value Model. 


\section{Introduction}

Linear dynamic rational expectation (RE) models admit an infinite number of dynamic equilibria that can be either stationary, or nonstationary. So far, the literature has focused on square integrable stationary (SIS) solutions and considered only specific nonstationary equilibria. It has been established that i) the RE model admits a unique SIS equilibrium only for some specific parameter values, while in general, there is an infinite number of SIS equilibria; ii) some nonstationary equilibrium paths feature explosive bubbles, usually explained by the self-fulfilling prophecies inherent to RE models.

This paper shows that, if the finite variance constraint on stationary solutions is relaxed, then there exists an infinite number of real valued or nonnegative stationary solutions to any linear rational expectation model. Note that the stationary solutions are in an uncertain environment the analogues of the constant or steady state solutions in deterministic framework. These stationary solutions with infinite variance include recurrent speculative bubbles and/or volatility induced mean-reversion. Their existence requires a new approach to the identification issue and to the impulse response analysis in linear RE models.

The paper is organized as follows. Section 2 reviews the literature on the set of equilibria in the RE model:

$$
y_{t}=a E_{t} y_{t+1}+z_{t},
$$

where $z_{t}$ represents the exogenous shocks, $E_{t}$ denotes the expectation conditional on the information available at date $t$ and $a$ is a scalar parameter. Section 2 also explains why the condition of finite variance is crucial for obtaining a unique stationary equilibrium when $|a|<1$. Section 3 provides the stationary solutions of the RE model with infinite variance. Two types of stationary nonlinear dynamics are discussed, one of which includes explosive bubbles and the other volatility induced mean-reversion. We also discuss the multiplicity when the solutions are constrained to be nonnegative. Section 4 discusses the identification of the stationary equilibrium and introduces a new approach to the impulse response analysis. Section 5 concludes. The Appendix provides a brief review of the dynamic bubble models considered in the literature and discusses the necessity of transversality conditions. 


\section{Set of Solutions}

This section describes the models and the solutions proposed in the existing economic literature.

\subsection{A rational expectation model}

For expository purpose, let us consider a simple textbook univariate linear rational expectation price model ${ }^{4}$ [see, Taylor (1977), Diba, Grossmann (1988), Flood, Hodrick (1990), Evans (1991)]:

$$
y_{t}=a E_{t}\left(y_{t+1}\right)+z_{t} .
$$

This model follows from an equilibrium model such as :

$$
\begin{cases}q_{t}=\alpha_{1} y_{t}+\alpha_{2} E_{t} y_{t+1}+z_{1 t}, & \text { (demand equation) } \\ q_{t}=\beta y_{t}+z_{2 t}, & \text { (supply equation) }\end{cases}
$$

where $q_{t}$ (resp. $y_{t}$ ) denotes the equilibrium quantity (resp. the equilibrium price), and $z_{1 t}, z_{2 t}$ represent the demand and supply shocks, respectively.

We assume that the joint process $\left(z_{1 t}, z_{2 t}\right)$ is strongly stationary and has a VARMA representation :

$$
\Phi(L)\left(\begin{array}{c}
z_{1 t} \\
z_{2 t}
\end{array}\right)=\Theta(L)\left(\begin{array}{l}
\varepsilon_{1 t} \\
\varepsilon_{2 t}
\end{array}\right)
$$

where $L$ denotes the lag operator, $\left(\varepsilon_{1 t}, \varepsilon_{2 t}\right)$ is a sequence of i.i.d. variables with finite variance and independent components, and the autoregressive polynomial is such that the roots of $\operatorname{det} \Phi(z)=0$ are outside the unit circle. The assumptions of normality and zero mean on $\epsilon_{t}$ are not required.

The equilibrium system (2.2) leads to model (2.1) with parameters:

$$
a=\frac{\alpha_{2}}{\beta-\alpha_{1}}, z_{t}=\frac{z_{1 t}-z_{2 t}}{\beta-\alpha_{1}} .
$$

\footnotetext{
${ }^{4}$ The results are easily extended to models with time-varying discount rate, multivariate models, models with more than one lag, and to expectations at different horizons [see e.g. Gourieroux, Laffont, Monfort (1982), Broze, Gourieroux, Szafarz (1995) for the description of the set of solutions in more general frameworks].
} 
As the equilibrium equation is obtained by matching the demand and supply, the information set contains the current and lagged values of the exogenous components and of the prices :

$$
I_{t}=\left(\underline{z_{1 t}}, \underline{z_{2 t}}\right)=\left(\underline{y_{t}}, \underline{z_{1 t}}, \underline{z_{2 t}}\right),
$$

where $\underline{y_{t}}=\left(y_{t}, y_{t-1}, y_{t-2}, \ldots\right)$, or equivalently $I_{t}=\left(\underline{\varepsilon_{1 t}}, \underline{\varepsilon_{2 t}}\right)=\left(\underline{y_{t}}, \underline{\varepsilon_{1 t}}, \underline{\varepsilon_{2 t}}\right)$.

This model provides also a rational expectation model for the equilibrium quantity:

$$
q_{t}=a E_{t}\left(q_{t+1}\right)+z_{2 t}+\beta z_{t}-a E_{t}\left(z_{2, t+1}\right),
$$

with the same coefficient $a$.

In this rational expectation model, both price and quantity variables take positive values. In a stochastic model, like this one, the equilibrium is an equilibrium of beliefs, that is an equilibrium of the conditional distributions of $y_{t+1}$ given $I_{t}$. In this respect, the outcome of the above model differs from that of a deterministic dynamic model, where the equilibrium is an equilibrium of prices and quantities. As the beliefs are subject to a few linear restrictions, a multiplicity of equilibria arises, due to the so-called selfconfirming belief (or self-fulfilling prophecy) [see e.g. Taylor (1977), Diba, Grossman (1988)a,b and Appendix 1].

\subsection{The linear SIS solutions}

The set of square integrable stationary (SIS) equilibrium prices depends on coefficient $a$ [see e.g. Blanchard (1978), Gourieroux, Laffont, Monfort (1982), Evans, Honkapohia (1986)]. If $|a|<1$, there is a unique SIS solution which

is defined as a linear moving average with an intercept: $y_{t}^{F} \equiv \sum_{j=0}^{\infty} A_{j} \varepsilon_{t-j}+b$. The process $y_{t}^{F}$ is called the forward solution, as for $|a|<1$, it can be obtained from the forward recursion:

$$
y_{t}^{F}=\sum_{h=0}^{\infty} a^{h} E_{t}\left(z_{t+h}\right) .
$$

If $|a|>1$, there is an infinite number of linear SIS solutions. They include the convex combinations of a solution with the same ARMA representation 
as $y_{t}^{F}{ }^{5}$ and of the perfect foresight (or backward) solution :

$$
y_{t}^{1}=a y_{t+1}^{1}+\varepsilon_{t} \Longleftrightarrow y_{t}^{1}=\frac{L}{L-a} \varepsilon_{t} .
$$

In model (2.1), the equilibrium solution has to be positive. For instance if $|a|<1$, the forward solution is admissible iff $\sum_{h=0}^{\infty} a^{h} E_{t} z_{t+h}>0$. This condition requires restrictions on parameter $a$ and on the dynamics of the exogenous variables. For example, the positivity of the forward solution is satisfied if $a>0, z_{t}>0$.

\subsection{The set of all solutions}

Gouriéroux, Laffont and Monfort (1982) have described the set of solutions of the RE model (2.1). These solutions can be written as:

$$
y_{t}=y_{t}^{F}+a^{-t} M_{t}
$$

where $M_{t}$ is a martingale satisfying : $M_{t}=E_{t} M_{t+1}$. Loosely speaking, process $\left(y_{t}\right)$ has two components that are the stationary process $\left(y_{t}^{F}\right)$ with linear dynamics and the martingale-based process $X_{t}=a^{-t} M_{t}$, which can feature nonlinear dynamics. Process $\left(X_{t}\right)$ satisfies $E_{t} X_{t+1}=a^{-1} X_{t}$ and is a submartingale, if $|a|<1$.

\subsection{Fundamental value and rational bubble}

Rational expectations also appear in asset pricing models. In that framework, model $(2.1)$ is $y_{t}=\left(1+r_{f}\right)^{-1} E_{t} y_{t+1}+z_{t}$, where $y_{t}$ is the value of the asset, ${ }^{6}$ which can be "resource, land, painting, jewel (for their consumption value)", or a "technology with decreasing returns to scale" [Tirole (1985)], $z_{t}$ the real rent (dividend), $r_{f}$ the riskfree rate assumed constant and where the information set includes various explanatory factors $z_{1 t}, z_{2 t}$ of the dividends.

\footnotetext{
${ }^{5}$ However this specific solution does no longer admit a forward interpretation, even if we still use the same notation $y_{t}^{F}$ [see e.g. Gourieroux, Laffont, Monfort (1982)].

${ }^{6}$ Model $(2.1)$ can also be seen as a Lucas-type model [Lucas (1978)] : $u^{\prime}\left(c_{t}\right) y_{t}=(1+$ $\left.r_{f}\right)^{-1} E_{t}\left[u^{\prime}\left(c_{t+1}\right)\left(y_{t+1}+d_{t+1}\right)\right]$. Then, model $(2.1)$ is valid with the product of value and marginal utility of consumption as the variable of interest, or to the value itself in the limiting case of a constant marginal utility (risk-neutral investor) (see subsection 3.3.i) and Appendix 3 for a deeper discussion of rational expectation models derived from models with intertemporal optimization).
} 
In such a case, $a=1 /\left(1+r_{f}\right)<1$, and the stationary linear solution is such that : $y_{t}^{F}=\sum_{h=0}^{\infty} \frac{1}{\left(1+r_{f}\right)^{h}} E_{t}\left(z_{t+h}\right)$. The corresponding $y_{t}^{F}$ is equal to the discounted sum of expected future dividends, and is called the fundamental value, or the present value [Shiller (1978), Flood and Garber (1980), Diba, Grossman (1988)], whereas the difference between the current value and the fundamental (present) value tautologically defines the rational bubble, or simply the bubble ${ }^{7}$. However, this interpretation of solution $y_{t}^{F}$ is no longer valid in the general equilibrium model (2.2), especially when $|a|>1$.

\subsection{Real-valued or nonnegative equilibria}

The dynamic RE model (2.1) with $|a|<1$ is a reduced form, which can be derived from a variety of structural models, such as the supply-demand equilibrium, an asset pricing model, a model of the debt-to-GDP ratio, or a first-order condition (Euler condition) to a life-cycle consumption model [Hall (1978)]. The underlying structural model determines the interpretation of the process $\left(y_{t}\right)$ and the information available at date $t$. For example, in the life cycle consumption model, $y_{t}$ is the marginal utility of consumption, $z_{t}=0$, and the information set includes the current and past observations on consumption, earning and asset variables except human capital.

Depending on the structural model, the equilibrium solution $\left(y_{t}\right)$ can be constrained to be nonnegative, or left unconstrained and taking real (positive or negative) values. For example, real-valued equilibria arise in the following applications:

i) In the asset pricing model of Section 2.4, where $y_{t}$ represents the value of a firm defined as the asset less liability, under an assumption that a firm is not automatically defaulted if the asset is less than the liability.

ii) When $z_{t}=0$, dynamic model (2.1) can also be applied to a selffinanced portfolio value under the risk-neutral probability. Without short-sell restrictions, this portfolio can take positive as well as negative values.

iii) Another example concerns the analysis of the balance of payments of different countries in RE models.

\footnotetext{
${ }^{7}$ It is also called the speculative bubble by other authors [see,e.g. Blanchard (1979), Blanchard, Fisher (1989), p. 218-221.] In our paper, we call "speculative" a bubble with explosive trajectory that ends with a burst.
} 
iv) In monetary models of speculative hyperinflation, there is a joint equilibrium on two markets: a consumption good market and a money market [see e.g. Brock (1975), Obstfeld, Rogoff (1983)]. This leads to three conditions on price level, real consumption and nominal money balance, respectively. Under simplifying assumptions, such as a constant consumption profile, two rational expectation equations are obtained, one for the path of the positive price level and another one for the real-valued money balance. In such a framework, one expects that the assumption of endogenous money leads to financial instability, bubbles and extreme volatility. Flat money in an overlapping generation model is probably the most famous example of bubble. The present value of flat money is zero; yet it has a positive price.

v) RE models are often specified in terms of changes of positive variables, without explicit references to any intertemporal optimization. The reason is to render the nominal variables more stationary. The changes can be of any sign. Similarly, the RE models can be directly written in log-transformed variables to eliminate the positivity restrictions [see, e.g. the so-called "adhoc" models in Sargent, Wallace (1975), the "generic canonical form" in Sims (2000), or the Cagan's model in Flood, Garber (1980)]. The RE models in logarithms of variables are often derived by log-linearization of a Dynamic Stochastic General Equilibrium (DSGE).

\section{$3 \quad$ Stationary Solutions with Infinite Variance}

The dynamic rational expectation equilibrium model (2.1) can have multiple stationary solutions even if $|a|<1$, when the stationary equilibrium price is not restricted to be square integrable. The notion of stationarity considered in this paper is the strong stationarity. Process $\left(y_{t}\right)$ is said to be strongly stationary iff the distribution of $\left(y_{t}, y_{t+1}, \ldots, y_{t+k}\right)$ is independent of $t$ for any $k \geq 0$. This notion does not require the existence of the first- and secondorder moments. The additional solutions include martingales which are nonintegrable, and therefore non-square-integrable too. Indeed, as shown below, the conditional expectation $E_{t} M_{t+1}$ may exist even when the unconditional expectation does not.

This section describes the stationary submartingales and explains why the RE models always have an infinite number of stationary solutions when these solutions are not constrained to be positive. That set of solutions is reduced under the positivity restriction, but is still infinite. 


\subsection{Stationary real-valued submartingales}

An integrable stationary submartingale is equal to zero almost surely. To show that, let us denote by $\left(X_{t}\right)$ the submartingale such that:

$$
E_{t}\left(X_{t+1}\right)=(1 / a) X_{t}, \text { with }|a|<1
$$

It follows that :

$$
E_{t}\left(\left|X_{t+1}\right|\right) \geq\left|E_{t}\left(X_{t+1}\right)\right|=(1 /|a|)\left|X_{t}\right|,
$$

and, by taking the expectation of both sides of the inequality :

$$
E\left|X_{t+1}\right| \geq(1 /|a|) E\left|X_{t}\right|
$$

Since $E\left|X_{t}\right|=E\left|X_{t+1}\right|$ by stationarity and $|a|<1$, this inequality implies $E\left|X_{t}\right|=0$, that is $X_{t}=0$ almost surely.

Equivalently, any nonzero stationary submartingale is necessarily nonintegrable (and has infinite variance). As mentioned above, when $|a|<1$, the process $X_{t}=a^{-t} M_{t}$ in (2.3) is a submartingale. Therefore, if the condition of stationarity of the solution is imposed, then $X_{t}$ (and $M_{t}$ ) is non-integrable. However we have at any horizon $h$ :

$$
E_{t}\left(X_{t+h}\right)=\frac{X_{t}}{a^{h}}
$$

Thus, if $X_{t} \neq 0$, the conditional expectations are explosive and no limit for $E_{t}\left(X_{t+h}\right)$ exists, when $h$ tends to infinity, but the conditional distribution of $X_{t+h}$ at date $t$ has a limit for stationary $\left(X_{t}\right)$. Below, we provide four examples of stationary ${ }^{8}$ non-integrable submartingales.

\subsubsection{Stable noncausal process}

A noncausal stable $\mathrm{AR}(1)$ is a strongly stationary process that satisfies the noncausal (or forward) autoregression [see Gourieroux, Zakoian (2016)]:

$$
y_{t}^{*}=\rho y_{t+1}^{*}+\varepsilon_{t}^{*}, 0 \leq \rho<1,
$$

\footnotetext{
${ }^{8}$ More precisely, processes $\left(y_{t}^{F}\right),\left(X_{t}\right)$ have to be jointly stationary.
} 
where the $\varepsilon_{t}^{*}$ are i.i.d. variables with a stable distribution ${ }^{9}$ with stability index $s, 0<s \leq 1$. This process is Markov in both the calendar and reverse times and admits a causal nonlinear autoregressive representation [see e.g. Rosenblatt (2000)] :

$$
y_{t}^{*}=g\left(y_{t-1}^{*}, \eta_{t} ; s, \rho\right),
$$

where $\left(\eta_{t}\right)$ is a strong Gaussian white noise, independent of the lagged values of $\left(y_{t}^{*}\right)$. The noise $\eta_{t}$ is an increasing function of $y_{t}^{*}$ for a given $y_{t-1}^{*}$, and is the Gaussian nonlinear innovation of Markov process $\left(y_{t}^{*}\right)$. Indeed, let us denote by $F\left(y_{t}^{*} \mid y_{t-1}^{*}\right)$ the conditional cumulative distribution function (c.d.f.) of $y_{t}^{*}$ given $y_{t-1}^{*}$. If function $F\left(. \mid y_{t-1}^{*}\right)$ is invertible, we know that $u_{t}=F\left(y_{t}^{*} \mid y_{t-1}^{*}\right)$ follows a uniform distribution on $[0,1]$. Thus $\eta_{t}=\Phi^{-1}\left(u_{t}\right)=$ $\Phi^{-1}\left[F\left(Y_{t}^{*} \mid y_{t-1}^{*}\right)\right]$, where $\Phi$ is the c.d.f. of the standard normal, follows $N(0,1)$, conditional on $y_{t-1}^{*}$. As $\left(y_{t}^{*}\right)$ is a Markov process, $\eta_{t}$ is also standard normal, conditional on $y_{t-1}^{*}=\underline{\eta_{t-1}}$. This implies the independence between $\eta_{t}$ and $\eta_{t-1}$, and the fact that $\overline{\left(\eta_{t}\right)}$ is a Gaussian white noise. The nonlinear autoregression (3.2) is obtained by inverting the relation that defines $\eta_{t}$.

As the reverse time innovation $\varepsilon_{t}^{*}$ has fat tails, $y_{t}^{*}$ does not have finite first and second-order unconditional moments. Its first and second-order moments conditional on the future are infinite as well. Nevertheless, it is shown in Gourieroux, Zakoian (2016) that this process has a first-order conditional moment given the past:

$$
E\left(y_{t+1}^{*} \mid \underline{y_{t}^{*}}\right)=\rho^{(s-1)} y_{t}^{*},
$$

where the autoregressive coefficient $\rho^{(s-1)}$ is larger than 1 . Thus this process is a stationary submartingale when $s<1$, a stationary martingale if $s=1$.

The path of a noncausal autoregressive stable process features local explosions followed by crashes. This is illustrated in Figure 1 by the simulated paths of noncausal autoregressive Cauchy processes with $\rho=0.5$ and $\rho=0.8$, respectively, and a Cauchy error $\varepsilon_{t}^{*}$. This corresponds to the limiting case of a martingale with $s=1$ for the stability index (and $a=1$ in the associated RE model).

[Insert Figure 1 : Path of Noncausal Stable Process]

\footnotetext{
${ }^{9} \mathrm{~A}$ stable distribution is a continuous distribution with characteristic function : $E[\exp (i u \varepsilon)]=\exp \left(-c|u|^{s}\right)$, where $c, c>0$, is a scale parameter. When $s=1$, we get the Cauchy distribution, when $s=1 / 2$ the Levy distribution.
} 
The positive or negative explosions cannot last indefinitely, as they are always followed by a crash. Whereas the bubble explodes in expectation, it is collapsing systematically in realization. ${ }^{10}$. When the above model is applied to the pricing of a monetary unit, the role of the money becomes a pure storage value [Samuelson (1958)]: "Temporarily money can yield a rate of interest lower than the market rate because it is used for transactions. But this effect must disappear sufficiently fast to allow a bubble" [Tirole (1985), p.1517]. Note that the simulated path shows that this process is compatible with "the reality where bubbles seem to deflate over several weeks or even months" [see the conclusion in Brunnermeier (2008)].

\subsubsection{Time discretized diffusion process}

Let us now consider the diffusion process defined by :

$$
d y_{t}^{*}=\delta y_{t}^{*} d t+\sigma\left(y_{t}^{*}\right) d W_{t},
$$

where $\left(W_{t}\right)$ is a Brownian motion, $\sigma($.$) the volatility function and \delta$ a positive scalar. Since $\delta$ is positive, this diffusion process has no (drift induced) mean reversion. This process satisfies the stationarity condition (more precisely the positive recurrence condition that implies ergodicity), if and only if, the two following conditions are satisfied [see Durett (1996), p221, or Kutoyants (2004), Th. 1.16] :

$$
\int_{0}^{x} \exp \left\{-2 \delta \int_{0}^{y} \frac{u}{\sigma^{2}(u)}\right\} d y \rightarrow \pm \infty, \text { as } x \rightarrow \pm \infty
$$

and

$$
\int_{-\infty}^{+\infty} \frac{1}{\sigma^{2}(y)} \exp \left(2 \delta \int_{0}^{y} \frac{u}{\sigma^{2}(u)} d u\right) d y<\infty
$$

\footnotetext{
${ }^{10}$ This example contradicts some largely diffused assertions, such as "The bubble process obeys the martingale equation...Clearly, the bubble component, if it exists, can never burst" [Montrucchio, Privileggi (2001) p. 165, and the discussion in Diba, Grossman (1988) b, Section III], or as "the bubble component will dominate the fundamental component" [Evans (1991), p.923]. This also question the notion of "growth" of the bubble component largely used in the literature. Indeed the sequence of predictions $E\left(y_{t+k}^{*} \mid y_{t}^{*}\right)$ explodes with $h$, at rate $\rho^{(s-1)}$, whereas the local explosions observed on the path of $y_{t}^{*}$ have a rate strictly larger than $\rho^{(s-1)}$, and the sequence of conditional distributions converges..
} 
Let us now assume that these conditions hold and consider the time discretized diffusion, that is the process $\left(y_{t}^{*}\right)$ observed at discrete dates $t=$ $1,2, \ldots$ This process is Markov in discrete time. We have :

$$
E_{t}\left(y_{t+d t}^{*}\right)=(1+\delta d t) y_{t}^{*} \simeq \exp (\delta d t) y_{t}^{*}
$$

and by iterated expectations, for a small $d t$ and a large number of iterations : $E_{t}\left(y_{t+1}^{*}\right)=(\exp \delta) y_{t}^{*}$. Thus, for $\delta$ such that $\exp \delta=1 / a$ with $0<a<1$, the process becomes a submartingale which can be added to the forward solution in order to obtain an additional new stationary solution to RE model (2.1), whenever the nonlinear innovation of $\left(y_{t}^{*}\right)$ is a function of $\left(\varepsilon_{1 t}, \varepsilon_{2 t}\right)$.

The path of the time discretized diffusion displays endogenous regimes of extremes, before reverting to more frequent values. In the literature, this pattern is called the volatility induced mean-reversion [see Conley et al. (1977)].

[Insert Figure 2 : Path of a Process with Volatility Induced Mean-Reversion]

This is illustrated in Figure 2 by the simulated paths of time discretized diffusion processes with $\delta=0, \sigma\left(y^{*}\right)=\sqrt{1+\left|y^{*}\right|}$ and $\sigma\left(y^{*}\right)=\sqrt{1+y^{* 2}}$, respectively, which correspond to the limiting case of a martingale (i.e. to $a=1$ in the associated RE model). When the process takes extreme values, the volatility becomes large. As a consequence, the process either remains extreme-valued in the future, or reverts to its frequently observed values. In general, clusters of extreme values are observed prior to the "mean reversion". Volatility induced mean reverting submartingales can also be constructed from autoregressive processes with ARCH effects. The basic model of this type is the double autoregressive model [see Borkovec, Kluppelberg (2001), Ling (2007), and Nielsen, Rahbek (2014) for an extension to multivariate processes.]

\subsubsection{A Stationary Martingale Tree}

The examples of stationary (sub)martingales given before may be difficult to interpret, especially for financial practitioners who are more familiar with the binomial trees a la Cox, Ross, Rubinstein (1979).

This subsection provides an example of a stationary submartingale tree. This tree features both speculative bubbles and volatility induced mean re- 
version. It also has deterministic dynamics in reverse time during the "explosive" bubble spell.

The process is defined as follows:

$$
y_{t+1}= \begin{cases}2 y_{t}+1, & \text { with probability } 1 / 4 \\ 2 y_{t}-1, & \text { with probability } 1 / 4 \\ +1, & \text { with probability } 1 / 4 \\ -1, & \text { with probability } 1 / 4\end{cases}
$$

where the regimes in each period are drawn independently. In some sense, the regime indicator plays the role of a shock at each time $t$.

For a given value $y_{t}$, we have:

$$
E\left(y_{t+1} \mid y_{t}=y\right)=\frac{1}{4}\left(2 y_{t}+1+2 y_{t}-1+1-1\right)=y_{t},
$$

which is the martingale condition. A stationary submartingale tree is obtained by replacing the scale factor 2 in the first step of the binomial tree by a larger scale factor.

This model can be interpreted as follows:

i) It can be considered as a binomial tree, which is observed every two periods. In the first period, we have:

$$
\tilde{y}_{t+1}= \begin{cases}2 y_{t}, & \text { with probability } 1 / 2, \\ 0, & \text { with probability } 1 / 2 .\end{cases}
$$

In the second period we get:

$$
y_{t+1}=\tilde{y}_{t+1}+\epsilon_{t+1},
$$

where $\epsilon_{t+1}$ takes values $+1,-1$, with equal probability $1 / 2$, and is independent of the regime indicator of the first period. Thus, the movement in the first period creates a potential explosion, or a mean reversion, whereas the movement in the second period is the standard dynamics found in the Cox, Ross, Rubinstein and Black, Scholes models.

ii) It can also be considered as an alternative to the bubble model proposed by Blanchard, Watson (1982) (see Appendix 2 ii)).

By construction the process can take odd values only, except for the first value. It regularly returns to +1 and -1 , due to the two last regimes in (3.7) (a recurrence property of this Markov chain). It can also display explosive trajectories in the set of values larger than 3 (resp. smaller than -3) during a 
period of "explosion". In order to describe this phenomenon, let us consider an initial value $y=3$ and all possible trajectories for the first two regimes in (3.7) (The results are similar for $y=-3$, say, and lead to a negative bubble).

At step $n, n=0,1,2, \ldots$, the odd numbers $2^{n+1}+1+2 m, m=0,1, \ldots, 2^{n}-$ 1 can be reached. All these trajectories are explosive. Their rate of explosion is stochastic, with an additive increase between $2^{n}$ and $2^{n+1}$ at each step $n$.

It is interesting to examine the martingale tree in reverse time. During an explosive bubble spell (either positive, or negative), the process is deterministic in reverse time while it is stochastic in calendar time. For instance, if the current observed value is $y=251$, say, the previous values are necessarily $125,63,31,15,7,3$. If the current observed value is 1233 , say, the previous values in calendar time are necessarily $617,309,155,77,39,19,9,5,3$.

The martingale tree is a Markov process, which is recurrent and stationary. Its stationary distribution is easily derived by using the property of deterministic bubble trajectories in reverse time.

First, we compute the marginal probabilities of +1 and -1 . We have:

$$
\begin{aligned}
P\left(y_{t+1}=1\right) & =P\left(y_{t+1}=1 \mid y_{t}=1\right) P\left(y_{t}=1\right)+P\left(y_{t+1}=1 \mid y_{t} \neq 1\right)\left[1-P\left(y_{t}=1\right)\right] \\
& =\frac{1}{2} P\left(y_{t}=1\right)+\frac{1}{4}\left[1-P\left(y_{t}=1\right)\right]=\frac{1}{4}+\frac{1}{4} P\left(y_{t}=1\right) .
\end{aligned}
$$

It follows that the stationary probability of 1 is:

$$
P\left(y_{t}=1\right)=1 / 3
$$

and by symmetry:

$$
P\left(y_{t}=-1\right)=1 / 3 \text {. }
$$

Next, we find the marginal probabilities of values in a positive bubble. We have:

$$
\begin{aligned}
P\left(y_{t+1}=3\right) & =\frac{1}{4} P\left(y_{t}=1\right)=\frac{1}{3} \frac{1}{4}, \\
P\left(y_{t+1}=5\right) & =\frac{1}{4} P\left(y_{t}=3\right)=\frac{1}{3}\left(\frac{1}{4}\right)^{2}, \ldots
\end{aligned}
$$

and so on. At step $n$ of the explosive bubble with positive values, we have:

$$
P\left(y_{t}=2^{n+1}+1+2 m\right)=\frac{1}{3} \frac{1}{4^{n+1}}
$$


It is easy to check that these probabilities sum up to 1 . Indeed the probabilities of positive values sum up to 0.5 as shown below:

$$
\begin{aligned}
P\left(y_{t}=1\right)+\sum_{n=0}^{\infty} \sum_{m=0}^{2^{n}-1} P\left(y_{t}=2^{n+1}+1+2 m\right) & =\frac{1}{3}+\sum_{n=0}^{\infty} \frac{1}{3} \sum_{m=0}^{2^{n}-1}\left(\frac{1}{4^{n+1}}\right) \\
& =\frac{1}{3}\left[1+\sum_{n=0}^{\infty}\left(\frac{2^{n}}{4^{n+1}}\right)\right] \\
& =\frac{1}{3}\left[1+\frac{1}{4} \sum_{n=0}^{\infty} \frac{1}{2^{n}}\right] \\
& =\frac{1}{3}\left(1+\frac{1}{4} \frac{1}{1-1 / 2}\right)=\frac{1}{3}\left(1+\frac{1}{2}\right)=\frac{1}{2} .
\end{aligned}
$$

In a similar way one can show that the probabilities of negative values sum up to 0.5 as well.

It is easy to check that the process features volatility induced mean reversion. Indeed, the change in the process is:

$$
\Delta y_{t+1}= \begin{cases}y_{t}+1, & \text { with probability } 1 / 4 \\ y_{t}-1, & \text { with probability } 1 / 4 \\ -y_{t}+1, & \text { with probability } 1 / 4 \\ -y_{t}-1, & \text { with probability } 1 / 4\end{cases}
$$

and the conditional variance is:

$$
V\left(y_{t+1} \mid y_{t}\right)=E\left[\left(\Delta y_{t+1}\right)^{2} \mid y_{t}\right]=\frac{1}{2}\left(y_{t}+1\right)^{2}+\frac{1}{2}\left(y_{t}-1\right)^{2}=y_{t}^{2}+1
$$

Thus, the larger $y_{t}^{2}$, the larger the variance. We conclude that this model displays volatility induced mean reversion towards 1 , or -1 .

In this simple framework:

i) We know with certainty if the process is in a phase of a positive (resp. negative) bubble, by checking if $y_{t} \geq 3$ (resp. $y_{t} \leq-3$ ).

ii) We can predict the time to bubble crash. Conditional on $y_{t} \geq 3$, we stay on a bubble with probability $1 / 2$, and we return to either \pm 1 , with probability $1 / 2$. Thus, this stochastic time to crash follows a Pascal distribution with parameter $1 / 2$. In particular, in this basic tree, the distribution of the 
time to crash does not depend on the time already spent in the bubble, contrary to the noncausal AR(1) Cauchy process, and the average sejourn time in a bubble is 2 .

As an illustration, Figure 3 shows a trajectory of such a process.

[Insert Figure 3: Path of the Stationary Martingale Tree]

\subsubsection{A Switching Regime Autoregression}

Let us consider two independent white noises $\left(z_{t}\right),\left(\varepsilon_{t}\right)$, where the $z_{t}^{\prime} s$ follow Bernoulli distributions $\mathcal{B}(1, p)$, and the $\varepsilon_{t}^{\prime} s$ are Gaussian with zero-mean and variance $\sigma^{2}$. The autoregressive model is defined by :

$$
y_{t}=\alpha \frac{z_{t}}{p} y_{t-1}+\varepsilon_{t}, \alpha>1, p<1,
$$

or equivalently at time $t, y_{t}$ is drawn in the distribution $N\left(\alpha y_{t-1}, \sigma^{2}\right)$ with probability $p$, and in the distribution $N\left(0, \sigma^{2}\right)$ with probability $1-p$. This autoregressive dynamics admits a stationary solution with the nonlinear moving average representation :

$$
y_{t}=\varepsilon_{t}+\frac{\alpha}{p} z_{t} \varepsilon_{t-1}+\left(\frac{\alpha}{p}\right)^{2} z_{t} z_{t-1} \varepsilon_{t-2}+\ldots
$$

Let us introduce the first time $\tau$ such that $z_{t-\tau}=0$. We have $P[\tau=$ $h]=(1-p) p^{h-1}, h \geq 1$. Moreover, when $\tau=h$, the variable $y_{t}$ is equal to

$y_{t}=\varepsilon_{t}+\frac{\alpha}{p} \varepsilon_{t-1}+\ldots+\left(\frac{\alpha}{p}\right)^{h-1} \varepsilon_{t-h+1}$. We deduce the stationary distribution of $y_{t}$ as the mixture of Gaussian distributions $N\left(0, \sigma^{2} \frac{1-(\alpha / p)^{2 h}}{1-(\alpha / p)^{2}}\right)$, with weights $(1-p) p^{h-1}$.

The conditional expectation of $y_{t}$ given $y_{t-1}$ is : $E\left(y_{t} \mid y_{t-1}\right)=\alpha y_{t-1}$, but the process has marginal fat tails. For instance, its unconditional variance is equal to :

$$
V\left(y_{t}\right)=\frac{\sigma^{2}(1-p)}{1-(\alpha / p)^{2}} \sum_{h=1}^{\infty} p^{h-1}\left[1-(\alpha / p)^{2 h}\right]=+\infty,
$$

since the general term of the series is of order $\left(\alpha^{2 h} / p^{h}\right)$, with $\alpha>1,1 / p>1$. 
The first-order moment $E\left(y_{t}\right)$ is also infinite, but the direct proof demands more computations.

Note that the probability of the regime does not depend on the current state $y_{t-1}$ to ensures the submartingale property. In this respect this switching regime is not a special case of STAR process [see Tong (1990), Bec et al. (2010)].

\subsection{Stationary nonnegative submartingales}

The stationary submartingales considered in Section 3.1 can take positive as well as negative values. Let us now focus on stationary submartingales constrained to take nonnegative values only. Examples of stationary nonnegative submartingales will be defined in an indirect way in Section 3.3. ii).

Lemma 1: For a stationary nonnegative martingale $\left(M_{t}\right)$, there exists a variable $M_{\infty}$ taking non-negative values and possibly the value $+\infty$, such that $M_{t}$ converges a.s. to $M_{\infty}$.

\section{Proof:}

Let us consider the process $X_{t}=\exp \left(-M_{t}\right)-1$. This process takes values between -1 and 0 , is uniformly integrable and such that:

$$
E_{t}\left(X_{t+1}\right)=E_{t}\left[\exp \left(-M_{t+1}\right)\right]-1 \geq \exp \left(-E_{t} M_{t+1}\right)-1=X_{t},
$$

by Jensen (convexity) inequality.

Next, we can apply Doob's martingale convergence theorem [see e.g. Williams (1991)]. There exists a variable $X_{\infty}$ such that $X_{t}$ converges a.s. and in $L^{1}$ to $X_{\infty}$. The result follows because the a.s. convergence also applies to the sequence $M_{t}$ with $M_{\infty}=-\log \left(1+X_{\infty}\right)$ Q.E.D.

The limit variable $X_{\infty}$ may take the values 0 and -1 with strictly positive probabilities. Then, $M_{\infty}$ may take the values 0 and $+\infty$.

Lemma 1 implies the following result :

Proposition 1: A nonnegative stationary solution to the equation $y_{t}=$ $a E_{t} y_{t+1}$, with $0<a<1$ is such that the limit of the associated martingale is $M_{\infty}=0$.

Proof: $M_{t}=a^{t} y_{t}$ is a nonnegative martingale. Therefore, it follows that $a^{t} y_{t} \rightarrow M_{\infty}$ a.s.. Since process $\left(y_{t}\right)$ is stationary, the marginal distribution 
of $\left(y_{t}\right)$ is equal to the marginal distribution of $\lim M_{\infty} / a^{t}$ (because the a.s. convergence implies the convergence in distribution). That "stationary distribution" is non-degenerate, iff there is no probability mass at $+\infty$, that is, if $M_{\infty}=0$ a.s. The result follows.

QED

\subsection{An infinite set of stationary equilibria}

The results of Sections 3.1-3.2 can be used to derive the set of stationary equilibria of RE model (2.1). We distinguish the case of real-valued and nonnegative solutions. We also discuss the common practice of log-linearization of DSGE models.

\section{i) Real-valued equilibria}

Let us return to the rational expectation model (2.1) with $0<a<1$, and introduce a standard Gaussian white noise $\eta_{t}$, which is a function of structural shocks $\varepsilon_{1 t}, \varepsilon_{2 t}{ }^{11}$. The stationary submartingales described in Section 3.1 can be used to construct an enlarged set of stationary solutions of the RE model (2.1) and more generally of any RE model. For expository purpose, let us consider the noncausal stable AR(1) process defined in Section 3.1.1 by :

$$
\begin{aligned}
y_{t}^{*}(s, \rho) & =g\left[y_{t-1}^{*}(s, \rho), \eta_{t} ; s, \rho\right] \\
\Leftrightarrow y_{t}^{*}(s, \rho) & =\rho y_{t+1}^{*}(s, \rho)+\varepsilon_{t}^{*}(s) .
\end{aligned}
$$

The process :

$$
y_{t}(s, \rho)=y_{t}^{F}+y_{t}^{*}(s, \rho)
$$

is a stationary solution to dynamic RE model (2.1), whenever :

$$
\rho=a^{1 /(1-s)} .
$$

as $y_{t}^{*}(s, \rho)$ is a submartingale with scale function $\rho^{s-1}=1 / a$, [see (3.3)].

This implies that, even if $0<a<1$, the $\mathbf{R E}$ equilibrium model (2.1) has an infinite number of stationary solutions. As the stability index $s$ can be chosen arbitrarily, by taking linear combinations of such submartingales, any process of the type :

\footnotetext{
${ }^{11}$ Such a standard noise can be obtained by selecting a function of $\varepsilon_{1 t}, \varepsilon_{2 t}: a_{t}=$ $a\left(\varepsilon_{1 t}, \varepsilon_{2 t}\right)$, say. Then $\eta_{t}$ is derived as $\eta_{t}=\Phi^{-1}\left[F_{a}\left[a_{t}\right]\right]=\Phi^{-1}\left[F_{a}\left[a\left(\varepsilon_{1 t}, \varepsilon_{2 t}\right]\right]\right.$, where $F_{a}$ denotes the c.d.f. of $a_{t}$, and $\Phi$ the c.d.f. of the standard normal
} 


$$
y_{t}=y_{t}^{F}+\Sigma_{s} \lambda(s) y_{t}^{*}\left[s, a^{1 /(1-s)}\right],
$$

is also a stationary solution of RE model (2.1). This contradicts Propositions 1 and 2 in Blanchard, Kahn (1980), for example.

The bubbles depend on the innovation of economic variables and have effects on the demand and supply. Thus, they are economically meaningful. These are intrinsic bubbles in the standard terminology [Froot, Obstfeld (1991), Brunnermeier (2008), Gurkaynak (2008)] and do not originate from self-confirming beliefs based on irrelevant variables, commonly called the sunspots (or animal spirits). 12

In practice, when $|a|<1$, it is common to select the "forward" solution as if it were the unique stationary solution of the rational expectation model. This practice disregards all stationary solutions with fat tails and nonlinear dynamics [see e.g. Pesaran, Smith (2011), p7, Leeper et al. (2013), eq. (4) and eq. (17), for recent examples of this practice, and the textbook by Ljunnqvist, Sargent (2000), Section 13.3 that reads: "In the general equilibrium model that we will describe later, the bubble term always equals zero."]

In the literature, the forward solution is often selected because of artificial constraints or approximations, which ensure that the process is integrable, and then are not innocuous. These constraints may be the consequence of a) borrowing constraints ${ }^{13}$ [see e.g. Ljungqvist, Sargent (2000), Chapter 8, 13, 17 , for the discussion of such constraints], or of b) a state space discretization leading to a finite state space [see e.g. Ljungqvist, Sargent (2000), Section 4.2 ], or of c) of distributional assumptions on the solution such as the lognormality [see e.g. Blanchard, Weil (2001), Section 4 for such an assumption in a storage economy], or of d) assumptions on the dynamics, such as a deterministic explosion rate [see e.g. Bertocchi (1991), eq. (4)].

\footnotetext{
${ }^{12}$ All these stationary solutions are intrinsic based on the smallest number of structural shocks. This constradicts the idea that bubble solution can be avoided by restricting attention to the "minimal state variable" solutions that depend only on fundamentals [McCallum (1983)].

${ }^{13}$ These artificial restrictions are often introduced to rule out (sustainable) Ponzi schemes. However, they also rule out other dynamics, as shown in the example of volatility induced mean-reverting process, or processes with explosive bubbles with burst. "It is not rare to find in the literature that some mechanism is called irrevelant because it is killed by the artificial constraints of the core. In many instances that can be corroborated by data, such results are really indictments of the artificial constraints, not of the mechanisms" [Caballero (2010)].
} 
Such implicit restrictions are also introduced to facilitate the estimation of parameters by instrumental variable [see e.g. Fuhrer, Oliver (2004), Gali et al. (2001), Dufour, Khalaf, Kichian (2006, (2010) for the so-called identification robust analysis of DSGE models or by Bayesian methods]. For instance let us consider the instrumental variable approach. Typically model (2.1) is rewritten as :

$$
y_{t}=a y_{t+1}+z_{t}+a \eta_{t+1}
$$

where the expectation error $\eta_{t+1}$ is such that $E_{t} \eta_{t+1}=0$, or equivalently parameter $a$ is defined by the conditional moment restriction :

$$
E_{t}\left[y_{t}-a y_{t+1}-z_{t}\right]=0,
$$

assuming for expository purpose that $z_{t}$ is observed. However, the implementation of a standard instrumental variable approach based on instruments $y_{t}$ and/or $z_{t}$, say, assumes the existence of cross moments such as $E\left(y_{t+1} y_{t}\right)$, and also the conditional homoscedasticity of the error term $V_{t} \eta_{t+1}=c t$. These assumptions are satisfied by the stationary integrable solution only, not by the nonlinear stationary solutions. Indeed the latter ones are non integrable and feature volatility effects. Other estimation approaches such as the maximum likelihood method or Bayesian approaches implicitly assume that a specific solution has been selected without justifying this choice [see e.g. Schorfheide (2015), Gallant, Giacomini, Ragusa (2014)].

The stationary solutions can take positive as well as negative values. These solutions are not ruled out by the basic absence of arbitrage opportunity condition. For example, let us consider a self-financed portfolio value $y_{t}$ such that $y_{t}=a E_{t}\left(y_{t+1}\right)$ and an investor selling (resp. buying) that portfolio at time $\mathrm{t}$ and buying (resp. selling) it back in the long run. Due to the stationarity of the bubble, we see that:

$$
\lim _{h \rightarrow \infty} P\left(y_{t+h}>y_{t} \mid y_{t}\right)=G\left(y_{t}\right),
$$

where $G$ is the c.d.f. of the stationary distribution of the process. For a noncausal process, a time-discretized diffusion, or the switching regime autoregression, this stationary distribution is continuous on $(-\infty,+\infty)$. Therefore, $G\left(y_{t}\right) \neq 0,1$ and there is no asymptotic arbitrage opportunity. ${ }^{14}$

\footnotetext{
${ }^{14} \mathrm{~A}$ similar result is valid when the selling time is a predetermined finite stopping time.
} 
The absence of arbitrage opportunity is satisfied in this case where the expected future value tends to infinity:

$$
\lim _{h \rightarrow \infty} E_{t}\left(y_{t+h}\right)=\lim _{h \rightarrow \infty} \frac{1}{a^{h}} y_{t}=\operatorname{sng}\left(y_{t}\right) \infty .
$$

This is due to the fact that the concept of arbitrage is not defined in mean-variance terms. Equivalently, the value represented by $y_{t}$ can diverge in mean $\left(L^{1}\right)$, without having an asymptotically explosive realized trajectory.

When model (2.1) is the set of Euler conditions in a Lucas-type economy, the existence of multiple stationary solutions with bubbles can also be explained as follows. As the demand is derived as a solution of an intertemporal optimization problem, it satisfies the Euler's conditions, as well as the transversality conditions. ${ }^{15}$ These latter conditions are often introduced in order to obtain a unique solution, that is to rule out the bubbles [see the discussion in Kamihigashi (2008)]. However, the transversality conditions may be not necessary. In particular, bubbles are not ruled out in a CCAPM model with unbounded utility [see Kamihigashi (1998), (2004) Montrucchio, Privileggi (2004) and Appendix 3].

To summarize, a multiplicity of real-valued stationary solutions to the RE model is obtained even when $|a|<1$ because of stationary (non integrable) submartingales. These stationary submartingales can be combined by choosing:

- the stability index for the noncausal stable submartingale and the dependence of nonlinear innovations on the exogenous shocks;

- the volatility function in the time discretized diffusion and the dependence of nonlinear innovations on the exogenous shocks;

- by introducing appropriate endogenous regimes in quadrinomial trees.

- or by considering different stationary and nonstationary switching regimes.

\footnotetext{
${ }^{15}$ Transversality conditions may appear when the agents are infinitely living, have the same preferences, and share the same information. They do not have to be introduced in overlapping generations models, under asymmetric information, under disagreement over the underlying value, or if there is limited liability [see e.g. Camerer (1989), Hong, Sraer (2013)]. Likely also, if there is a finite, but imprecisely known market depth [see Enders, Hakenes (2014) for a study of this framework].
} 
In Lucas type model these solutions are rational, i.e. they are compatible with agents maximizing their intertemporal utility.

The multiplicity of solutions is specific to stochastic models. Indeed, in the associated deterministic model: $y_{t}=a y_{t+1}+z$, say, all bubbles are nonstationary. They never burst, once they start growing.

\section{ii) Nonnegative equilibria}

As mentioned earlier, the equilibria must be nonnegative when the variable of interest is a price, or the quantity of a physical good. Stationary patterns usually characterize quantities. But price processes, such as commodity prices, exchange rates, or real prices may have stationary patterns too.

The set of stationary equilibria is reduced under the nonnegativity restriction, but remains infinite.

Indeed, let us first consider a RE model with a constant negative $z$ :

$$
y_{t}=a E_{t} y_{t+1}+z, 0<a<1, z<0 .
$$

Note that a model with negative $z$ may be appropriate for the dynamics of a commodity price, where $z$ is interpreted as a convenience yield, i.e. a cost of storage.

Lemma 2 : Model (3.15) has an infinite number of positive stationary solutions.

Proof : i) First note that the forward solution $y_{t}^{0 F}=\frac{z}{1-a}$ is negative and thus is not admissible.

ii) Another solution is the process defined by :

$$
y_{t+1}^{0}=\left\{\begin{array}{l}
\frac{1}{a p} y_{t}^{0}, \text { with probability } p, 0<p<1, \\
-\frac{z}{a(1-p)}, \text { with probability } 1-p,
\end{array}\right.
$$

where the regime indicator is based on the latent innovations ${ }^{16}\left(\varepsilon_{1, t+1}, \varepsilon_{2, t+1}\right)$.

\footnotetext{
${ }^{16}$ There latent innovations may come from the exogenous processes with effects on demand and supply, even if a specific linear combination of these exogenous processes, i.e. $z$, is constant.
} 
This process is positive, such that :

$$
E_{t} y_{t+1}^{0}=\frac{1}{a} y_{t}^{0}-\frac{z}{a}
$$

This process is stationary, with stationary distribution :

$$
P\left[y_{t}=\frac{-z}{a(1-p)} \frac{1}{(a p)^{n}}\right]=(1-p) p^{n}, n=0, \ldots,
$$

and not integrable.

iii) Other real valued solutions are obtained by considering the combinations :

$$
y_{t}(\lambda, z, p)=\lambda y_{t}^{0}+(1-\lambda) y_{t}^{0 F}
$$

Since $y_{t}(\lambda, z, p)=\lambda y_{t}^{0}+(1-\lambda) \frac{z}{1-a}>\frac{-\lambda z}{a(1-p)}+\frac{(1-\lambda) z}{1-a}$,

this combination is a positive solution, when $\lambda>\frac{a(1-p)}{1-a p}$.

To summarize we get an infinite set of positive stationary solutions parametrized by $\lambda$ and $p$.

QED

Let us now consider the model :

$$
y_{t}=a E_{t} y_{t+1}+z_{t}, z_{t} \geq 0,
$$

where the nonnegative process $z_{t}$ is stationary integrable. This corresponds to the model of Section 2.4. For this model the forward solution given by :

$$
y_{t}^{F}=\sum_{h=0}^{\infty} a^{h} E_{t} z_{t+h},
$$

is now positive and then an admissible solution.

Proposition 2 : Model (3.16) has an infinite number of nonnegative stationary solutions.

Proof : Let us denote : 


$$
x_{t}(z, p)=y_{t}(1, z, p)-y_{t}(0, z, p)=y_{t}^{0}-y_{t}^{0 F}, z<0,0<p<1 .
$$

This process is a positive stationary solution of the homogenous REmodel : $x_{t}(z, p)=a E_{t} x_{t+1}(z, p)$.

Therefore $y_{t}^{F}+x_{t}(z, p)$ is a positive stationary solution of model (3.14). This set of solutions is parametrized by $z$ and $p, z<0,0<p<1$.

QED

Let us now discuss consequences of Proposition 2. For expository purpose, we denote by $\left(\varepsilon_{t}\right)$ an i.i.d. process taking values 1 and 3 with probability $p=1 / 2$, choose $z_{t}=1-\varepsilon_{t}$, and use $\left(\varepsilon_{t}-1\right) / 2$ as the indicator function for defining the bubble process $y_{t}^{0}-y_{t}^{0 F}$. Since $E_{t}\left(z_{t+h}\right)=-1$, the forward solution is :

$$
y_{t}^{F}=1-\varepsilon_{t}-\frac{a}{1-a},
$$

whereas a solution with bubble is :

$$
\begin{aligned}
y_{t} & =y_{t}^{F}+y_{t}^{0}-y_{t}^{0 F} \\
& =1-\varepsilon_{t}-\frac{a}{1-a}+\frac{2}{a} y_{t-1}^{0} \frac{\varepsilon_{t}-1}{2}-\frac{2 z}{a}\left(1-\frac{\varepsilon_{t}-1}{2}\right)-\frac{z}{1-a} \\
& =c t+\varepsilon_{t}\left(-1+\frac{y_{t-1}^{0}}{a}+\frac{z}{a}\right) .
\end{aligned}
$$

The bubble equilibrium is not integrable and is a nonlinear function of $\underline{\varepsilon_{t}}$ by means of $y_{t}^{0}$. Nevertheless both $y_{t}^{F}$ and $y_{t}$ have a conditional variance given the past equal to $\sigma_{\varepsilon}^{2}$ and $\sigma_{\varepsilon}^{2}\left(1-\frac{y_{t-1}^{0}}{a}-\frac{z}{a}\right)^{2}$, respectively. We immediately note that $\left(1-\frac{y_{t-1}^{0}}{a}-\frac{z}{a}\right)^{2}$ cannot be larger than 1 for any admissible value $y_{t}^{0}$ and any negative $z$. Thus, when nonlinear stationary solutions are introduced, the forward solution is not the solution with minimum conditional variance [see a discussion of this criterion in Taylor (1977)]. The reason is the following one : the bubble component is based on the same innovation 
as the forward solution and can be negatively conditionally correlated with the forward solution.

\section{iii) Log-linearization of DSGE models}

It is common practice to log-linearize the equilibrium conditions derived from the DSGE models ${ }^{17}$ [see, e.g. Boragan et al. (2006) , Christiano et al. (2010) for examples]. The approach consists in replacing the (positive) variables by their log-expansion about a steady state $\mu$, say, that is, in approximating $y_{t}$ by:

$$
y_{t} \sim \mu+\mu\left(\log y_{t}-\log \mu\right)
$$

and next, replacing the initial model (2.1):

$$
y_{t}=a E_{t} y_{t+1}+z_{t}
$$

by the log-linearized model:

$$
\log y_{t}=a E_{t} \log y_{t+1}+z_{t} / \mu+(1-a)(\log \mu-1) .
$$

This log-linearized approximation is not innocuous in the search for stationary solutions of the DSGE model, even though the stationarity of $y_{t}$ is equivalent to the stationarity of $\log y_{t}$.

In the log-linearized version (3.17), the variable $\log y_{t}$ can be of any sign, resulting in an infinite number of stationary solutions, whereas in the initial model (2.1), in which the variable is positive, the number of solutions is still infinite, but smaller. This also implies that numerical procedures designed for finding "the" stationary solution to the log-linearized model (3.17) become questionable. The existing algorithms automatically select a unique solution and rely on numerical arguments rather than on either economic, or statistical arguments. 18

\footnotetext{
${ }^{17}$ or from dynamic stochastic partial equilibrium models.

${ }^{18}$ The same remarks apply to the log-linearized versions of the present value model [see e.g. Campbell, Shiller (1989)].
} 


\section{Identification of the Equilibrium and Im- pulse Response Analysis}

This section discusses the consequences of a possibly infinite set of stationary solutions of RE model (2.1). Given the multiplicity of solutions, the outcomes of numerical algorithms that select a unique solution need to be taken with caution. We address now the identification of the stationary equilibrium and the impulse response analysis.

At the beginning, we explain why the available observations can be used to statistically identify the stationary solution. Next, we point out that the dynamics of the stationary solution may be nonlinear, which requires a nonlinear approach to impulse response analysis.

Concerning the identification of the solution, our result differs from the recent literature [see e.g. Iskrev (2010), Komunjer, Ng (2011)]. The reason is that we prove the identification of a stationary solution from the initial model, whereas the literature usually considers the solution identification from the log-linearized vector autoregressive approximation of the initial model. Moreover, our approach is not a second-order identification, but instead a distribution-based identification. We use the assumption of i.i.d. errors in order to identify the nonlinear component of the dynamics.

\subsection{Identification}

In Section 3, we showed that the RE model (2.1) with $|a|<1$ has an infinite number of stationary solutions, which are derived from the forward solution by adding stationary submartingales of various types. These submartingales are Markov processes:

$$
y_{t}^{*} \equiv h\left(y_{t-1}^{*}, a\left(\varepsilon_{1 t}, \varepsilon_{2 t}\right)\right),
$$

where $h$ is a nonlinear transformation and $a\left(\varepsilon_{1 t}, \varepsilon_{2 t}\right)$ is a Gaussian noise derived from the innovations of exogenous variables that appear in the demand and supply. Under standard stability conditions such a process admits also a nonlinear infinite moving average representation :

$$
y_{t}^{*} \equiv H\left[\varepsilon_{1 t}, \varepsilon_{2 t}, \varepsilon_{1, t-1}, \varepsilon_{2, t-1}, \ldots\right],
$$

which can itself be written as a series expansion when $H$ satisfies appropriate 
differentiability conditions ${ }^{19}$. That is the Volterra expansion of the process written in terms of the i.i.d. sequence $\left(\epsilon_{t}\right)$ [see e.g. Priestley (1981), Volterra (2005)] :

$$
\begin{aligned}
y_{t}^{*}= & b+\sum_{h=0}^{\infty} a_{1 h}^{\prime} \varepsilon_{t-h}+\sum_{h=0}^{\infty} \sum_{k=0}^{\infty} a_{2 h k}^{\prime} \operatorname{vec}\left(\varepsilon_{t-h} \varepsilon_{t-k}^{\prime}\right) \\
+ & \sum_{\substack{h=0 \\
h \leq k}}^{\infty} \sum_{\substack{k=0 \\
h}}^{\infty} \sum_{l=0}^{\infty} a_{3 h k l}^{\prime} \operatorname{vec}\left[\operatorname{vec}\left(\varepsilon_{t-h} \varepsilon_{t-k}^{\prime}\right) \varepsilon_{t-l}^{\prime}\right]+\ldots,
\end{aligned}
$$

where $\varepsilon_{t}=\left(\varepsilon_{1 t}, \varepsilon_{2 t}\right)^{\prime}$ and vec $A$ denotes the vector obtained by stacking the columns of matrix $A$.

If both the forward solution and the additional submartingales have Volterra expansions, then the solution of the RE model also has a Volterra expansion.

The reason why multiple stationary rational expectation equilibria have been disregarded in the past literature was that the Volterra expansion is written only for linear stationary solutions and was therefore restricted to have zero moving average coefficients on terms of orders larger than 2 : $a_{2 h k}=0, a_{3 h k l}=0, \ldots$, Hence, all stationary solutions with nonlinear dynamics, such as speculative bubbles, volatility induced mean-reversion, and/or switching regimes have been artificially eliminated.

The joint model for $\left(y_{t}, z_{t}\right)$, where $y_{t}$ is the price and $z_{t}$ is an exogenous process (resp. dividend) also admits a Volterra expansion in i.i.d. errors $\left(\varepsilon_{1 t}, \varepsilon_{2 t}\right)$. Let us now discuss how to identify the stationary solution from an observed long trajectory of the joint process $\left(y_{t}, z_{t}\right)$, that is how to derive consistent approximations of the moving average coefficients $a_{1 h}, a_{2 h k}, a_{3 h k l}, \ldots$ and polynomials $\Phi$ and $\Theta$ that characterize the dynamics of $z_{t}$ (see equation

\footnotetext{
${ }^{19}$ These differentiability conditions are likely satisfied for the processes considered in Section 3.1.1-3.2.1. They do not hold for the basic exogenous innovations in the stationary martingale tree of Section 3.1.3. In this latter case, the additional submartingale may be of the type:

$$
y_{t+1}=\left(2 y_{t}+1\right) 1_{\epsilon_{1 t} \leq a_{1}}+\left(2 y_{t}-1\right) 1_{a_{1} \leq \epsilon_{1 t} \leq a_{2}}+1_{a_{2} \leq \epsilon_{1 t} \leq a_{3}}-1_{\epsilon_{1 t}>a_{3}},
$$

where $a_{j}, j=1,2,3$ are the quartiles of the distribution of $\epsilon_{1 t}$, assuming the impact of the exogenous innovation through $\varepsilon_{1 t}$, only. We get a moving average expansion which involves the indicator transformations of $\epsilon_{1 t}$.
} 
(2.3)). This identification problem is common in the time series literature on moving averages:

$$
y_{t}=A_{0} \epsilon_{t}+A_{1} \epsilon_{t-1}+A_{2} \epsilon_{t-2}+\cdots,
$$

that do not contain products of innovations. If the $\epsilon_{t}$ 's are i.i.d. and if $\epsilon_{1 t}$ is independent of $\epsilon_{2 t}$, we can identify matrix coefficients $A_{0}, A_{1}, \ldots$ up to a permutation of indexes 1,2 , of the $\epsilon$ 's and a multiplicative scale factor [see, e.g. Chan, Ho, Tong (2006)].

This identification result can be extended to nonlinear moving averages, i.e. to the Volterra expansion [see e.g. Christensen, Trott (1969), Koukoulas, Kalouptsidis (1995)]. Thus in such a RE model with multiple stationary equilibria, the RE equilibrium chosen by the agents can be identified from the joint observed paths of the price $y$ and exogenous variable $z$. That is, it is not necessary for identification to introduce any additional restriction leading to a unique stationary equilibrium.

The intuition underlying identification is that the econometrician and the economic agents have different information sets. More precisely, the econometrician observes ex-post $\left(y_{t}, z_{t}\right), t=1, \ldots, T$ over the whole period as well as the consequences of agents' decisions at all times, whereas at time $t$ the agents know only the past values of the variable up to time $t$. If $T$ is large, $T \rightarrow \infty$ and $\left(y_{t}\right)$ is strictly stationary, it is possible to estimate nonparametrically the nonlinear regression of $y_{t+1}$ on the past values $y_{t}, y_{t-1}, \ldots z_{t}, z_{t-1}, \ldots$ and next to estimate consistently parameter $a$ by regressing $y_{t}$ on $z_{t}$ and $\hat{y}_{t \mid t+1}$, where $\hat{y}_{t \mid t+1}$ denotes the predicted value. The difference with the standard instrumental variable approach, which implicitly selects the linear stationary solution, is the following : in the IV approach the expectation $E_{t} y_{t+1}$ is replaced by a linear prediction based on the instruments. Thus it differs from the true RE except for the linear stationary solution. Moreover this error in variable is not necessarily independent of the exogenous shocks. By considering nonparametric prediction, the error is asymptotically zero for any stationary solution. Of course this identification result says nothing about how the agents behaviours and expectations are coordinated to reach this identified collective rational equilibrium.

The identification result above and the associated semi-parametric estimation method could be difficult to apply in finite sample, especially to macroeconomic data. In the next subsection, we consider a parametric infinite set of stationary solutions constructed from the forward solution and a 
noncausal stable submartingale in order to illustrate the implementation of nonlinear impulse response analysis.

\subsection{Nonlinear IRF for a SVAR Model with Noncausal Component}

Let us assume $|a|<1$. The impulse response analysis describes how structural shocks propagate through the macroeconomy. The approach depends on the selected solution and differs from the standard linear analysis that is based on the forward solution only. The reason is that it has to take into account the nonlinear dynamics of the stationary submartingale. To illustrate this effect let us consider a solution with a noncausal stable submartingale :

$$
y_{t}(s, \rho)=y_{t}^{F}+y_{t}^{*}(s, \rho),
$$

and assume $z_{t}=\varepsilon_{1 t}-\varepsilon_{2 t}$, where $\varepsilon_{t}=\left(\varepsilon_{1 t}, \varepsilon_{2 t}\right)^{\prime} \sim N(0, I d)$. The forward solution is $y_{t}^{F}=z_{t}=\varepsilon_{1 t}-\varepsilon_{2 t}$.

Equation (3.5) implies that:

$$
\begin{aligned}
& y_{t}(s, \rho)=\frac{\varepsilon_{t}^{*}(s, \rho)}{1-\rho L^{-1}}+\varepsilon_{1 t}-\varepsilon_{2 t} \\
\Leftrightarrow \quad & (L-\rho) y_{t}(s, \rho)=L \varepsilon_{t}^{*}(s)+(L-\rho)\left(\varepsilon_{1 t}-\varepsilon_{2 t}\right) .
\end{aligned}
$$

The VARMA representation of $\left(y_{t}, z_{t}\right)$ written in terms of $\varepsilon_{t}^{*}(s), \varepsilon_{1 t}-\varepsilon_{2 t}$ :

$$
\left\{\begin{aligned}
(L-\rho) y_{t}(s, \rho)-(L-\rho) z_{t} & =L \varepsilon_{t}^{*}(s), \\
z_{t} & =\varepsilon_{1 t}-\varepsilon_{2 t},
\end{aligned}\right.
$$

is such that one root of the autoregressive polynomial lies inside the unit circle. In particular $\left(\varepsilon_{t}^{*}(s), \varepsilon_{1 t}-\varepsilon_{2 t}\right)$ is not the causal linear innovation of $\left(y_{t}(s, \rho), z_{t}\right)$.

The standard Box-Jenkins approach applied to the bivariate series $\left[y_{t}(s, \rho), z_{t}\right]$ is invalid for the following reasons : First, the Box-Jenkins method assumes the square integrability of $y_{t}(s, \rho)$, whereas this process has no mean. Second the Box-Jenkins approach is a linear approach, which cannot accommodate the nonlinear innovation $\left(\eta_{t}\right)$ in $(3.2)$.

The impulse response functions of a one-time shock at time $t$, represent the effects of shocks on $\left(\varepsilon_{1 t}-\varepsilon_{2 t}, \eta_{t}\right)^{\prime}$ (where $\left.\eta_{t}=a\left(\varepsilon_{1 t}, \varepsilon_{2 t}\right)\right)$, rather 
than on errors $\left[\varepsilon_{1 t}-\varepsilon_{2 t}, \varepsilon_{t}^{*}(s)\right]$, as $\varepsilon_{t}^{*}(s)$ is a nonfundamental error. This means that the impulse responses should not be based on the nonfundamental VARMA representation (4.2), but instead on the associated nonlinear causal autoregressive representation. Since bubbles are driven nonlinearly by the exogenous fundamentals, changes in these fundamentals can cause prices to overreact.

More precisely system (4.2) has to be rewritten in terms of the causal innovations as :

$$
\begin{aligned}
y_{t}(s, \rho) & =y_{t}^{F}+y_{t}^{*}(s, \rho), z_{t}=\varepsilon_{1 t}-\varepsilon_{2 t}, \\
\text { where }: y_{t}^{F} & =z_{t}=\varepsilon_{1 t}-\varepsilon_{2 t}, \\
y_{t}^{*}(s, \rho) & =g\left[y_{t-1}^{*}(s, \rho), a\left(\varepsilon_{1 t}, \varepsilon_{2, t}\right), s, \rho\right],
\end{aligned}
$$

and where $g$ and $a$ are given nonlinear functions.

The IRF are computed from stochastic shocks to structural errors $\varepsilon_{1 t}, \varepsilon_{2 t}$, by applying the nonlinear recursive scheme (4.3)-(4.5) [see Koop et al. (1996), Gourieroux, Jasiak (2005) for nonlinear IRF] and running nonlinear recursions. The shock can be either deterministic, or stochastic, and can possibly correspond to stressed situations [see Gourieroux, Jasiak (2015) for simulation of noncausal processes].

\section{Concluding Remarks}

A linear RE equilibrium model with current expectations always admits an infinite set of real-valued or nonnegative stationary dynamic equilibria even when $|a|<1$. These multiple intrinsic equilibria are derived from the forward solution and include stationary submartingales. These submartingales can feature speculative bubbles that explode and burst, volatility induced mean reversion and/or switching regimes. The existence of multiple stationary nonlinear equilibria requires an adequate analysis of identification, and a revised approach to impulse response analysis in RE models in order to account for the nonlinearities. The multiplicity of stationary equilibria challenges the current practices and principles of macroeconomic modeling, such as: 
-the assumption of a unique stationary equilibrium;

-the numerical algorithms that provide a specific solutions based on numerical criteria only;

-the idea that log-linearization is innocuous;

-the view that an explosive bubble will last indefinitely and dominate the fundamental solution;

- the idea that the forward solution has the minimal conditional variance; -the opinion that a RE model and its perfect-foresight analogue produce similar results;

-the estimation of parameters by standard instrumental variable approach, maximum likelihood, or Bayesian techniques. 
Moreover the possibility of stationary (non integrable) bubble component also challenges the main testing procedures of the present value model introduced in the literature. Let us consider these testing procedures.

i) The cointegration test [Diba, Grossman (1987)] is based on the idea that prices will be more nonstationary than the dividends, if there is a nonzero bubble component. This argument is no longer valid if the bubble is stationary.

ii) The variance bounds test introduced by Leroy, Porter (1981) [see also Cochrane (1992)] assumes the existence of the marginal variance of the price $y_{t}$. This assumption is not satisfied if the bubble component is stationary, then non integrable. We have also proved that a solution with bubble could be less volatile than the solution with zero bubble.

iii) The two step procedure introduced by West (1987) [see also Dezbakash, Demirgue-Kunt (1990)] is based on a standard instrumental variable estimation of the discount factor from the Euler restrictions. We mentioned in Section 3.3 that these standard estimation approaches are no longer valid. 


\section{Appendix 1}

\section{Equilibrium of Beliefs}

\section{i) Stochastic RE model}

For expository purpose, let us consider a stationary Markov process that satisfies the RE condition:

$$
y_{t}=a E_{t} y_{t+1} .
$$

Let us denote by $f\left(y_{t+1} \mid y_{t}\right)$ the transition pdf and $g\left(y_{t}\right)$ the marginal (stationary) pdf. The unknown transition pdf has to satisfy the following restrictions:

unitary mass restriction: $\int f\left(y_{+1} \mid y\right) d y_{+1}=1, \forall y$,

$\int y_{+1} f\left(y_{+1} \mid y\right) d y_{+1}=y / a, \forall y$.

This "number" of restrictions is much smaller than the number of possible transitions, which leaves room for an infinite number of solutions, i.e. of belief equilibria.

However, the stationarity assumption implies that the transition pdf has also to satisfy the Kolmogorov equation. There exists a function $g$ such that:

$$
\int f\left(y_{+1} \mid y\right) g(y) d y=g\left(y_{+1}\right), \forall y_{+1},
$$

where $\int g(y) d y=1$ and $g(y) \geq 0, \forall y$.

These inequality restrictions on function $g$ might considerably reduce the number of admissible stationary solutions. Note that the positivity restrictions concern the stationary pdf, not the process itself.

\section{ii) Deterministic perfect foresight model}

This outcome is very different from the outcome of its deterministic analogue: $y_{t}=a y_{t+1}$. In that case, the solutions are written in terms of process $\left(y_{t}\right)$. These are equilibria of trajectories, and no longer equilibria of beliefs. These solutions are given by $y_{t}=y_{0} / a^{t}, t \geq 0$, where $y_{0}$ is a (possibly stochastic) initial value. If $0<a<1$, we get the unique stationary solution with the choice of $y_{0}=0$, that is $y_{t}=0, \forall t$. 


\section{Appendix 2}

\section{Explosive Bubble Models in the Literature}

Explosive bubbles are dynamic patterns that represent a short (stochastic) explosion followed by a crash. Several examples of (sub)martingales with explosive bubbles were considered in the economic literature or RE models.

We discuss below the most commonly used models of bubble dynamics that appeared in the literature. These models usually present martingale processes, corresponding to the case $a=1, z_{t}=0$.

\section{i) Blanchard (1979).}

The bubble dynamics is defined by:

$$
Y_{t+1}=\left\{\begin{array}{cl}
\frac{1}{\pi} Y_{t}, & \text { with probability } \pi, \\
0 & \text { with probability } 1-\pi .
\end{array}\right.
$$

This process represents a single bubble, with 0 as an absorbing state. The rate of explosion of the bubble is fixed and equal to $1 / \pi$. It is strictly larger than the average rate of explosion, which is equal to 1 by the martingale condition.

The regime indicator appearing in the bubble model above may be defined as a function of the standard Gaussian nonlinear innovation $\left(\epsilon_{t}\right)$ of the exogenous process (see Gouriéroux, Laffont and Monfort (1982), p.42), that leads to the model:

$$
Y_{t+1}=\frac{1}{\pi} Y_{t} \mathbb{1}_{\epsilon_{t+1}<\Phi^{-1}(\pi)},
$$

where $\Phi$ is the c.d.f. of the standard normal.

In an alternative specification, the indicator variable may alse be assumed as independent of the innovation process. Hence, two different choices of the information set are available.

The bubble has been considered as a martingale component to be added to the forward solution in order to obtain an additional solution of RE model (2.1). However, the martingales in the above model are nonstationary and asymptotically tend to zero. Therefore they induce no long term effects of self-fulfilling prophecies.

ii) Blanchard and Watson (1982). 
This bubble model eliminates zero as an absorbing state. The dynamics of the bubble is given by:

$$
Y_{t+1}= \begin{cases}\frac{1}{\pi} Y_{t}+\epsilon_{t+1}, & \text { with probability } \pi, \\ \epsilon_{t+1}, & \text { with probability } 1-\pi,\end{cases}
$$

where, for instance, $\left(\epsilon_{t}\right)$ is an i.i.d. zero mean sequence constructed from the nonlinear Gaussian innovation of the exogenous process. This process takes positive as well as negative values. As before, the regime indicator can be a function of $\left(\epsilon_{t}\right)$, or it can be independent of the innovation process. This model allows for erratic changes in $Y_{t}$ during the explosion spell. It also allows for multiple explosive bubbles. When $\varepsilon_{t} \sim N\left(0, \sigma^{2}\right)$ this process is a limiting case of the switching process analyzed in Section 3.1.4, corresponding to $a=1$.

\section{iii) Evans (1991).}

Evans proposed an explosive bubble with a stochastic rate of explosion:

$$
Y_{t+1}= \begin{cases}u_{t+1} Y_{t}, & \text { if } Y_{t}<\alpha \\ \left(\delta+\frac{1}{\pi} \theta_{t+1}\left(Y_{t}-\delta\right)\right) u_{t+1}, & \text { if } Y_{t}>\alpha\end{cases}
$$

where $\alpha, \delta$ are parameters such that $0<\delta<\alpha,\left(u_{t}\right)$ is an i.i.d. process with $u_{t} \geq 0, E_{t}\left(u_{t+1}\right)=1$ for all $t,\left(\theta_{t}\right)$ is an i.i.d. Bernoulli process $\mathcal{B}(1, \pi)$. As long as $Y_{t}<\alpha$, the bubble erupts at a faster mean rate $1 / \pi$, but bursts with probability $1-\pi$. The model requires an information set strictly larger than the set generated by the exogenous innovation process.

\section{iv) Kamihigashi (2011)}

The dynamics is defined by :

$$
\log Y_{t+1}=\mu\left(Y_{t}\right)+\varepsilon_{t+1} / c\left(Y_{t}\right)
$$

where the $\varepsilon_{t}^{\prime} s$ are i.i.d. with a symmetric distribution. Then it is proved (see Corollary 5.1 in Kamihigaski (2011) that the process is recurrent if

- function $c$ is decreasing from $(0, \infty)$ to $(0, \infty)$, with $0<c(\infty) \leq c(0)<$ $\infty$,

and

- $\mu(\infty)<0<\mu(0)$. 
The recurrence property ensures that the process will pass an infinite number of times in any neighbourhood of any positive real value. In other words the process will neither explode with probability 1, nor asymptotically tend to 0 with probability 1 . The recurrence property does not imply the strong stationarity of the process. ${ }^{20}$ For instance the random walk without drift corresponds to the limiting case : $c(y)=c$ constant, $\mu(y)=0$, is recurrent and nonstationary. To get the stationarity additional conditions have to be introduced. More precisely it is known that any recurrent Markov process has a stationary distribution with density $f(y)$, say, which corresponds to a positive measure. Thus $f(y)>0$, but does not necessarily sum up to one. This limiting density is the solution of the Kolmogorov's equation :

$$
f(y)=\int_{0}^{\infty} \frac{c(z)}{y} g\{c(z)[\log y-\mu(z)]\} f(z) d z,
$$

where $g$ denotes the density of the noise $\varepsilon$.

Thus the strong stationarity of the process requires the following necessary condition :

$$
\int_{0}^{\infty} f(y) d y=1 \text {, i.e. the unit mass restriction, }
$$

plus additional stability conditions.

From the current discrete time literature the conditions on $p$ and $c$ to get the strong stationarity are not yet known.

\section{Appendix 3}

\section{Necessity of Transversality Conditions}

Before analyzing the potential role of transversality conditions in our framework, let us first remark that

i) the main part of the literature on transversality conditions focuses on deterministic optimizations [see e.g. Michel (1990), Ekeland, Scheinkman (1986), Kamihigashi (2001), (2008)], and cannot be immediately extended to optimization in a stochastic environment.

\footnotetext{
${ }^{20}$ See Gourieroux, Jasiak (2016) for the test of the martingale hypothesis under the recurrence assumption.
} 
ii) Moreover, as mentioned in Kamihigashi (2001), "the Euler equation is derived from the optimality requirement that no gain be achieved by choosing a path that deviates from the optimal path, but eventually returns to it. By contrast the transversality condition is derived from the requirement that no gain be achieved by choosing a path that deviates from it forever". In our case the stationary bubble components create paths that will not deviate forever.

Let us now consider a standard Consumption based Capital Asset Pricing Model (CCAPM) to show that the transversality conditions do not eliminate the stationary bubbles. The intertemporal optimization problem is :

$$
\max _{\left(\alpha_{t}, C_{t}\right)} \sum_{t=0}^{\infty} \beta^{t} E_{0} U\left(C_{t}\right), \text { s.t. } C_{t}+\alpha_{t} p_{t}=R_{t}+\alpha_{t-1} p_{t}, \forall t
$$

$C_{t}$ is the consumption at date $t$ with the price of the consumption good fixed to 1 for expository purpose, $\alpha_{t}$ the quantity of risky asset, $p_{t}$ its unitary price $\left(p_{t}>0\right), R_{t}, R_{t}>0$ and exogenous income, $U$ the utility function and $\beta$ a discount, $0<\beta<1$.

After eliminitating the current and future budget constraints, we get the intertemporal optimization :

$$
\max _{\left(\alpha_{t}\right)} \sum_{t=0}^{\infty} \beta^{t} E_{0} U\left[R_{t}+p_{t}\left(\alpha_{t-1}-\alpha_{t}\right)\right] .
$$

The optimum $\left(\alpha_{t}^{*}\right)$ (if it exists) satisfies the Euler restrictions :

$$
p_{t} U^{\prime}\left[R_{t}+p_{t}\left(\alpha_{t-1}^{*}-\alpha_{t}^{*}\right)\right]=\beta E_{t}\left[p_{t+1} U^{\prime}\left[R_{t+1}+p_{t+1}\left(\alpha_{t}^{*}-\alpha_{t+1}^{*}\right)\right]\right\}, \forall t,
$$

and also the inequalities :

$$
C_{t}^{*}=R_{t}+p_{t}\left(\alpha_{t-1}^{*}-\alpha_{t}^{*}\right)>0, \forall t .
$$

Let us now assume the equilibrium on the financial market :

$$
\alpha_{t}^{*}=\alpha_{0 t}, \forall t
$$

where the asset supply $\alpha_{0 t}$ is exogenous and assumed to decrease :

$$
\alpha_{0, t-1}>\alpha_{0, t}(>0)
$$


This condition implies that the sequence $\alpha_{0, t}$ will tend to a positive limit when $t$ tends to infinity, and also that $\alpha_{t-1}^{*}-\alpha_{t}^{*}=\alpha_{0, t-1}-\alpha_{0, t}$ will tend to zero.

Under assumption (a.6) the inequalities (a.5) are automatically satisfied.

Let us now consider a stationary positive submartingale $\left(y_{t}\right)$ satisfying :

$$
y_{t}=\beta E_{t} y_{t+1} .
$$

Lemma : Let us consider a CRRA utility function :

$U(C)=C^{\gamma} / \gamma$, with $0<\gamma<1$. There exists (at least) a positive process $\left(p_{t}\right)$ solution of :

$$
p_{t} U^{\prime}\left[R_{t}+p_{t}\left(\alpha_{0, t-1}-\alpha_{0, t}\right)\right]=y_{t}, \forall t
$$

Proof :

Indeed the function of $p_{t}$ in the left hand side is equal to :

$$
G\left(p_{t}\right)=\gamma p_{t} /\left[R_{t}+p_{t}\left(\alpha_{0, t-1}-\alpha_{0, t}\right)\right]^{1-\gamma} .
$$

This function is such that : $G(0)=0, G(\infty)=\infty$. This provides the result.

\section{QED}

When $t$ tends to infinity, this solution is equivalent to :

$$
p_{t} \sim \frac{1}{\gamma} R_{t}^{1-\gamma} y_{t}
$$

Is this solution compatible with the intertemporal optimization problem, in particular with the "transversality conditions"? The answer is yes. Indeed, the utility function $U$ is unbounded, and we can apply Proposition 4.3 in Kamihigashi (2005) :

if $\sum_{t=1}^{\infty} \beta^{t} E_{0} U\left[R_{t}+p_{t}\left(\alpha_{0, t-1}-\alpha_{0, t}\right)\right]<\infty$, the transversality conditions are automatically satisfied. Otherwise, the lifetime utility is infinite at the optimum and the transversality conditions are not necessary. 
As noted in Kamihigashi (2005), p 1323: "This case is usually ruled out in practice and (the conditional finite lifetime utility) assumed implicitly or taken for granted in applied studies". This practice can eliminate important solutions.

How to explain this larger number of solutions ? Of course the limiting case : $\alpha_{0, t}=\alpha_{0}$ independent of time, would not be compatible with the equilibrium on financial market. Indeed the investor would have always the same quantity of asset, and no need to trade for updating his/her portfolio. In our example, there are trades at any finite dates, since the number of shares in the portfolio diminishes (but not necessarily the value of the portfolio), and the floating will tend to zero. From an economic viewpoint the multiplicity of solutions is likely due to the fact that the virtual financial market at $t=\infty$ cannot exist.

From a mathematical viewpoint, the multiplicity of stationary solutions satisfying the positivity and the standard conditions for the optimization is likely due to the fact that the value of the objective function at the optimum is infinite. Indeed the stationary bubbles are not integrable. In such a framework, it is not possible to select among these solutions on the basis of the value of the intertemporal expected utility only. This problem has already been encountered in a deterministic framework for the Ramsey's model of economic growth. In this framework the idea is to choose between the solutions by considering the speed at which the intertemporal utilities up to a finite time $T$, say, tend to infinity, when $T$ tends to infinity. In other words the objective function has to be modified. Such a change of criterion appears for instance in the notion of sustainable development [see e.g. Chichilnisky (1996), Asheim, Ekeland (2016)]. In the stochastic framework, the deterministic time $T$ would have to be replaced by a sequence $\tau_{T}$ of (stochastic) stopping times tending to $\infty$ with $T$. Such an analysis and the possible economic interpretations of such new criteria are beyond the scope of the present paper. 


\section{References}

[1] Anderson, G. (2008): "Solving Linear Rational Expectations Models: A Horse Race", Computational Economics, 31, 95-113.

[2] Asheim, G., and I., Ekeland (2016) : "Resource Conservation Across Generations in a Ramsey-Chichilnisky Model", forthcoming Economic Theory.

[3] Bec, F. Ben Salem, M., and M., Carrasco (2010) : "Detecting Mean Reversion in Real Exchange Rates from a Multiple Regime STAR Model", Annals of Economics and Statistics, 99/100, 395-422.

[4] Bertocchi, G. (1991): "Bubbles and Inefficiencies", Economic Letters, 35, 117-122.

[5] Black, F. (1974) : "Uniqueness of the Price Level in Monetary Growth Models with Rational Expectations", Journal of Economic Theory, 7, 5365 .

[6] Blanchard, O. (1978) : "Backward and Forward Solutions for Economics with Rational Expectations", DP 627, Harvard University.

[7] Blanchard, O. (1979) : "Speculative Bubbles, Crashes and Rational Expectations", Economic Letters, 3, 387-389.

[8] Blanchard, O., and S. Fisher (1989): "Lectures on Macroeconomics", MIT Press.

[9] Blanchard, O., and C. Kahn (1980): "The Solution of Linear Difference Models Under Rational Expectations", Econometrica, 48, 1305-1312.

[10] Blanchard, O. J. and M. W. Watson (1982): Bubbles, Rational Expectations and Financial Markets. In Crises in the Economic and Financial Structure, ed. by P. Wachtel, 295-316. Lexington, MA: D.C. Heathand Company.

[11] Blanchard, O. and P. Weil (2001): "Dynamic Efficiency, the Riskless Rate and Debt Ponzi Games Under Uncertainty", in Advances in Macroeconomics, Vol. 1, Berkeley Electronic Press. 
[12] Boragan, A., Fernandez-Villaverde, J., and J. Rubio-Ramirez (2006): "Comparing Solution Methods for Dynamic Equilibrium Economies", Journal of Economic Dynamics and Control, 30, 2477-2508.

[13] Borkovec, M. and C. Kluppelberg (2001): "The Tail of the Stationary Distribution of an Autoregressive Process with ARCH(1) Errors", Annals of Applied Probability, 11, 1220-1241.

[14] Brock, W. (1975): "A Simple Perfect Foresight Monetary Model", Journal of Monetary Economics, 1, 133-150.

[15] Broze, L., Gourieroux, C., and A., Szafarz (1995) : "Solutions of Multivariate Rational Expectations Models", Econometric Theory, 11, 229-257.

[16] Brunnermeier, M. (2008) : "Bubbles", in S., Durlauf and L. Blume, eds, New Palgrave Dictionary of Economics, 2nd edition, Palgrave MacMillan, London.

[17] Caballero, R. (2010) : "Macroeconomics After the Crisis : Time to Deal with the Pretense of Knowledge Syndrome", Journal of Economic Perspective, 24, 85-102.

[18] Camerer, C. (1989): "Bubbles and Fads in Asset Prices", Journal of Economic Surveys, 3, 3-45.

[19] Campbell, J., and R., Shiller (1989) : "The Dividend-Price Ratio and Expectations of Future Dividends and Discount Factors", Review of Financial Studies, 1, 195-228.

[20] Chan, K., Ho, L. and H. Tong (2006): "A Note on Time Reversibility of Multivariate Linear Processes", Biometrika, 93, 221-227.

[21] Chichilnisky, G. (1996) : "An Axiomatic Approach to Sustainable Development", Soc. Choice Welfare, 13, 231-257.

[22] Christiano, L., Trabandt, M. and K. Walentin (2010): "DSGE Models for Monetary Policy Analysis", NBER 16074.

[23] Christensen, G., and G., Trott (1969) : "On the Uniqueness of the Volterra Series", IEEE Trans. Aut. Control, 14, 759-760. 
[24] Cochrane, J. (1992) : "Explaining the Variance of Price Dividend Ratios", Review of Financial Studies, 5, 243-280.

[25] Conley, T., Hansen, L., Luttmer, E., and J., Scheinkman (1997) : "Short Term Interest Rates as Subordinated Diffusions", Review of Financial Studies, 10, 525-577.

[26] Cox, J., Ross, S. and M. Rubinstein (1979): "Option Pricing: A Simplified Approach", Journal of Financial Economics, 7, 229-263.

[27] Dezbakhash, H., and A., Demirgue-Kunt (1990) : "On the Presence of Speculative Bubbles in Stock Prices", The Journal of Financial and Quantitative Analysis, 25, 101-112.

[28] Diba, B., and H., Grossman (1987) : "On the Inception of Rational Bubbles", Quarterly Journal of Economics, 87, 697-700.

[29] Diba, B., and H., Grossman (1988)a: "Explosive Rational Bubbles in Stock Prices ?", The American Economic Review, 78, 520-530.

[30] Diba, B. and H. Grossman (1988)b "The Theory of Rational Bubbles in Stock Prices", The Economic Journal, 98, 746-754

[31] Dufour, J.M., Khalaf, L., and M., Kichian (2006) : "Inflation Dynamics and the New Keynesian Phillips Curve : An Identification Robust Econometric Analysis", Journal of Economic Dynamics and Control, 30, 1707-1727.

[32] Dufour, J.M., Khalaf, L., and M., Kichrian (2010) : "IdentificationRobust Analysis of DSGE and Structural Macroeconomic Models", Journal of Monetary Economics, 60, 340-350.

[33] Durett, R. (1996) : "Stochastic Calculus : A Practical Introduction", Boca Raton, CRC Press.

[34] Ekeland, I., and J., Scheinkman (1986) : "Transversality Conditions for Some Infinite Horizon Discrete Time Optimization Problems", Mathematics of Operations Research, 11, 216-219.

[35] Enders, Z., and H., Hakenes (2014) : "On the Existence and Prevention of Speculative Bubbles", Univ. of Heidelberg, DP 567. 
[36] Evans, G. (1991): "Pitfalls in Testing for Explosive Bubbles in Asset Prices", The American Economic Review, 81, 922-930.

[37] Evans, G. and S. Honkapohia (1986): " A Complete Characterization of ARMA Solutions to Linear Rational Expectation Models", Review of Economic Studies, 53, 227-239.

[38] Flood, R., and P., Garber (1980): "Market Fundamentals Versus Price Level Bubbles. The First Tests", Journal of Political Economy, 88, 745770 .

[39] Flood, R., and R., Hodrick (1990) : "On Testing for Speculative Bubbles", Journal of Economic Perspectives, 4, 85-101.

[40] Froot, K., and M., Obstfeld (1991) : "Intrinsic Bubbles : The Case of Stock Prices", American Economic Review, 81, 1189-1214.

[41] Fuhrer, J., and G., Oliver (2004) : "Estimating Forward Looking Euler Equations with GMM Estimators : An Optimal Instruments Approach", Technical Report, Federal Reserve Bank of Chicago.

[42] Gali, J., Gerthler, M., and J., Lopez-Salido (2001) : "European Inflation Dynamics", European Economic Review, 45, 1327-1270.

[43] Gourieroux, C., and J., Jasiak (2005) : "Nonlinear Impulse Response Functions", Annales d'Economie et de Statistique, 78, 1-33.

[44] Gourieroux, C., and J., Jasiak (2015) : "Filtering, Prediction and Simulation Methods for Noncausal Processes", Journal of Time Series Analysis, 37, 405-430.

[45] Gourieroux, C., and J., Jasiak (2016) : "Robust Analysis of the Martingale Hypothesis", CREST DP.

[46] Gourieroux, C., Laffont, J.J., and A., Monfort (1982) : "Rational Expectation in Dynamic Linear Models : Analysis of the Solutions", Econometrica, 50, 409-425.

[47] Gourieroux, C., and J.M., Zakoian (2016) : "Explosive Bubble Modelling by Noncausal Process", forthcoming Journal of the Royal Statistical Society. 
[48] Gurkaynak, R. (2008) : "Econometric Tests of Asset Price Bubbles : Taking Stock", Journal of Economic Surveys, 22, 166-186.

[49] Hall, R. (1978): "Stochastic Implications of the Life Cycle Permanent Income Hypothesis: Theory and Evidence", Journal of Political Economy, 86, 971-988.

[50] Harrison, M., and D., Kreps (1979) : "Martingales and Arbitrage in Multiperiod Securities Markets", Journal of Economic Theory, 20,0381408.

[51] Hong, H., and D., Sraer (2013) : "Quiet Bubbles", Journal of Financial Economics, 110, 596-606.

[52] Iskrev, N. (2010): "Local Identification in DSGE Models", Journal of Monetary Economics, 57, 189-202.

[53] Kamihigashi, T. (1998): "Uniqueness of Asset Prices in an Exchange Economy with Unbounded Utility", Economic Theory, 12, 103-122.

[54] Kamihigashi, T. (2001) : "Necessity of Transversality Conditions for Infinite Horizon Problems", Econometrica, 69, 995-1012.

[55] Kamihigashi, T. (2004) : "Necessity of the Transversality Condition for Stochastic Models with Bounded or CRRA Utility", Journal of Economic Dynamics and Control, 29, 1313-1329.

[56] Kamihigashi, T. (2008): "Transversality Conditions and Dynamic Economic Behavior", in New Palgrave Dictionary of Economics, 2nd edition, ed. Durlauf, S. and Blume, L.

[57] Kamihigashi, T. (2011) : "Recurrent Bubbles", Japanese Economic Review, 62, 27-62.

[58] Klein, P. (2000): "Using the Generalized Schur Form to Solve a Multivariate Linear Rational Expectations Model", Journal of Economic Dynamics and Control, 24, 1405-1423.

[59] Komunjer, I., and S. Ng, (2011): "Dynamic Identification of DSGE Models", 79, 1995-2032. 
[60] Koop, G., Pesaran, M., and S., Potter (1996) : "Impulse Response Analysis in Nonlinear Multivariate Models", Journal of Econometrics, 74, 119-147.

[61] Koukoulas, P., and N., Kalouptsidis (1995) : "Nonlinear System Identification Using Gaussian Inputs", IEEE Trans. Signal Process., 43, 18311841 .

[62] Kutoyants, Y. (2004) : "Statistical Inference for Ergodic Processes", Springer.

[63] Leeper, E., Walker, T., and S., Yang (2013) : "Fiscal Foresight and Information Flows", Econometrica, 81, 1115-1145.

[64] LeRoy, S., and R., Porter (1981) : "The Present-Value Relation : Tests Based on Implied Variance Bounds", Econometrica, 49, 555-574.

[65] Ling, S. (2007): "A Double AR(1) Model: Structure and Estimation", Stat. Sin., 17, 161-175.

[66] Ljungqvist, L. and T. Sargent (2000): "Recursive Macroeconomic Theory", Cambridge MIT Press.

[67] Lucas, R. (1978): "Asset Prices in an Exchange Economy", Econometrica, 46, 1429-1445.

[68] McCallum, B. (1983) : "On Non-Uniqueness in Rational Expectations Models : An Attempt at Perspective", Journal of Monetary Economics, $11,139-168$.

[69] Miao, J. (2004) : "Introduction to Economic Theory of Bubble", Journal of Mathematical Economics, 53, 130-136.

[70] Michel, P. (1990) : "Some Clarifications on the Transervasilty Condition", Econometrica, 58, 705-723.

[71] Montrucchio, L. and F. Privileggi (2001): "On Fragility of Bubbles in Equilibrium Asset Pricing Models of Lucas-Type", Journal of Economic Theory, 101, 158-188. 
[72] Nielsen, H. and A. Rahbek (2014): "Unit Root Vector Autoregression with Volatility Induced Stationarity", Journal of Empirical Finance, 29, 144-167.

[73] Obstfeld, M. and K. Rogoff (1983): "Speculative Hyperinflations in Maximizing Models: Can We Rule Them Out", Journal of Political Economy, 91, 675-687.

[74] Pesaran, H., and R., Smith (2011) : "Beyond the DSGE Straitjacket", DP 1138, Cambridge University.

[75] Priestley, M. (1981) : "Spectral Analysis and Time Series", Academic Press, San Diego.

[76] Rosenblatt, M. (2000) : "Gaussian and Non Caussian Linear Time Series and Random Fields", Springer Verlag.

[77] Samuelson, P. (1985): "An Exact Consumption Loan Model of Interest with or without the Social Contrivance of Money", Journal of Political Economy, 66, 467-482.

[78] Sargent, T., and N. Wallace (1975): "Rational Expectations, the Optimal Monetary Instrument and the Optimal Money Supply Rule", Journal of Political Economy, 83, 241-254.

[79] Schorfheide, F. (2011) :"Estimation and Evaluation of DSGE Models : Progress and Challenges", DP Federal Reserve Bank of Philadelphia.

[80] Shiller, R. (1978): "Rational Expectation and the Dynamic Structure of Macroeconomic Models: A Critical Review", Journal of Monetary Economics, 4, 1-49.

[81] Sims, C. (2002): "Solving Linear Rational Expectations Models", Computational Economics, 20, 1-20.

[82] Taylor, J. (1977) : "Condition for Unique Equilibrium in Stochastic Macroeconomic Models with Rational Expectation", Econometrica, 45, $1372-1385$.

[83] Tirole, J. (1985): "Asset Bubbles and Overlapping Generations", Econometrica, 53, 1499-1528. 
[84] Tong, H (1990) : "Nonlinear Time Series", Oxford Univ. Press.

[85] Velinov, A., and W., Chen (2013) : "Are There Bubbles in Stock Prices? Testing for Fundamental Shocks", DP 1375, DIW, Berlin.

[86] Volterra, V. (2005) : "Theory of Functionals and of Integrand and Integrodifferential Equations", Dover, New-York.

[87] West, K. (1987) : "A Specification Test for Speculative Bubbles", The Quarterly Journal of Economics, 102, 553-580.

[88] Williams, D. (1991): "Probability with Martingale", Cambridge University Press. 
Noncausal Cauchy AR(1), coeff $=0.5$

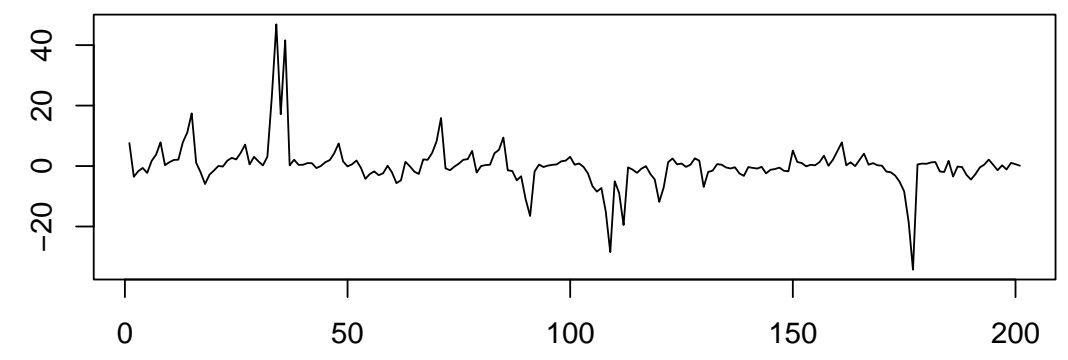

Noncausal Cauchy AR(1), coeff $=0.8$

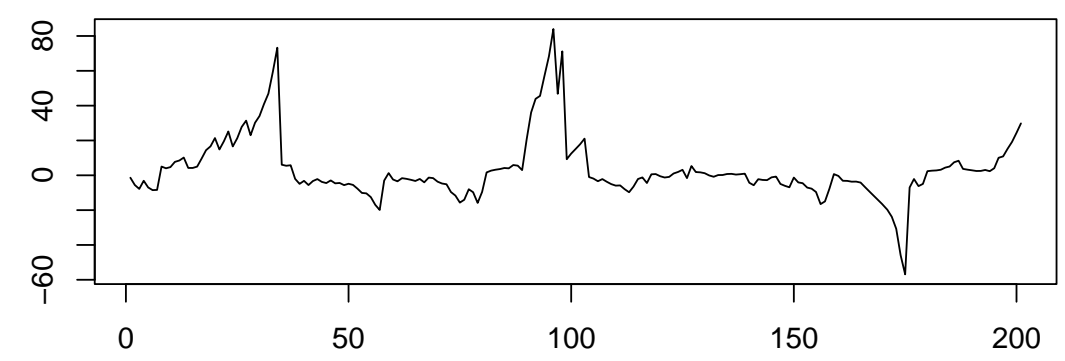

Figure 1: Path of a Noncausal Stable Process 


\section{Diffusion a)}

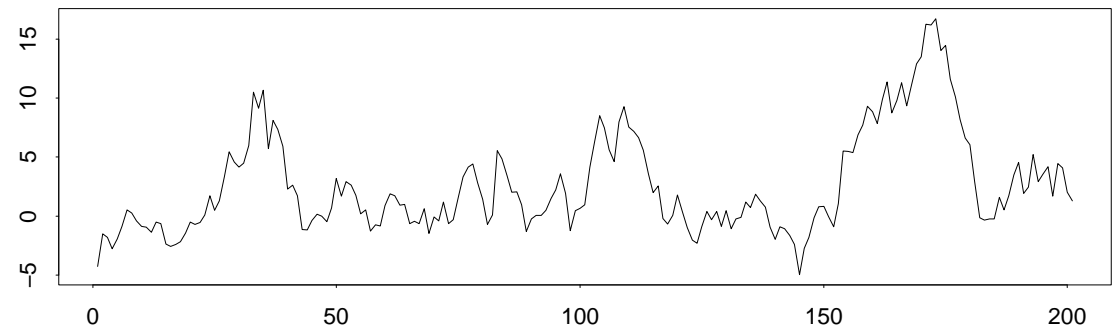

Diffusion b)

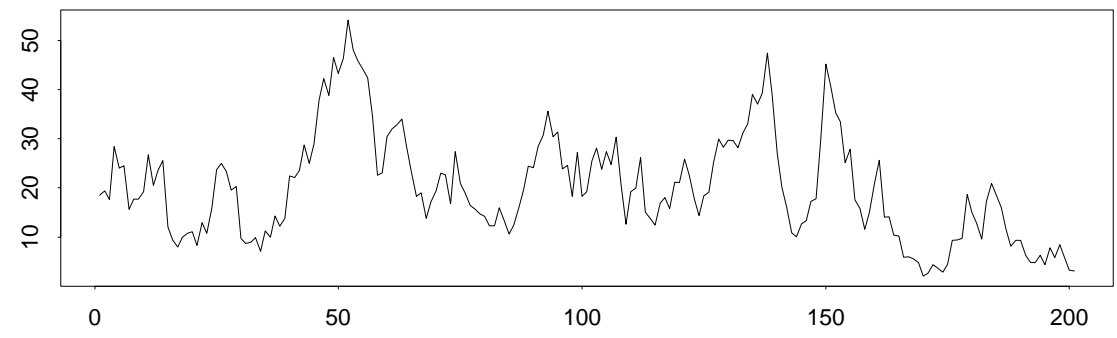

Figure 2: Path of a Process with Volatility Induced Mean-Reversion 


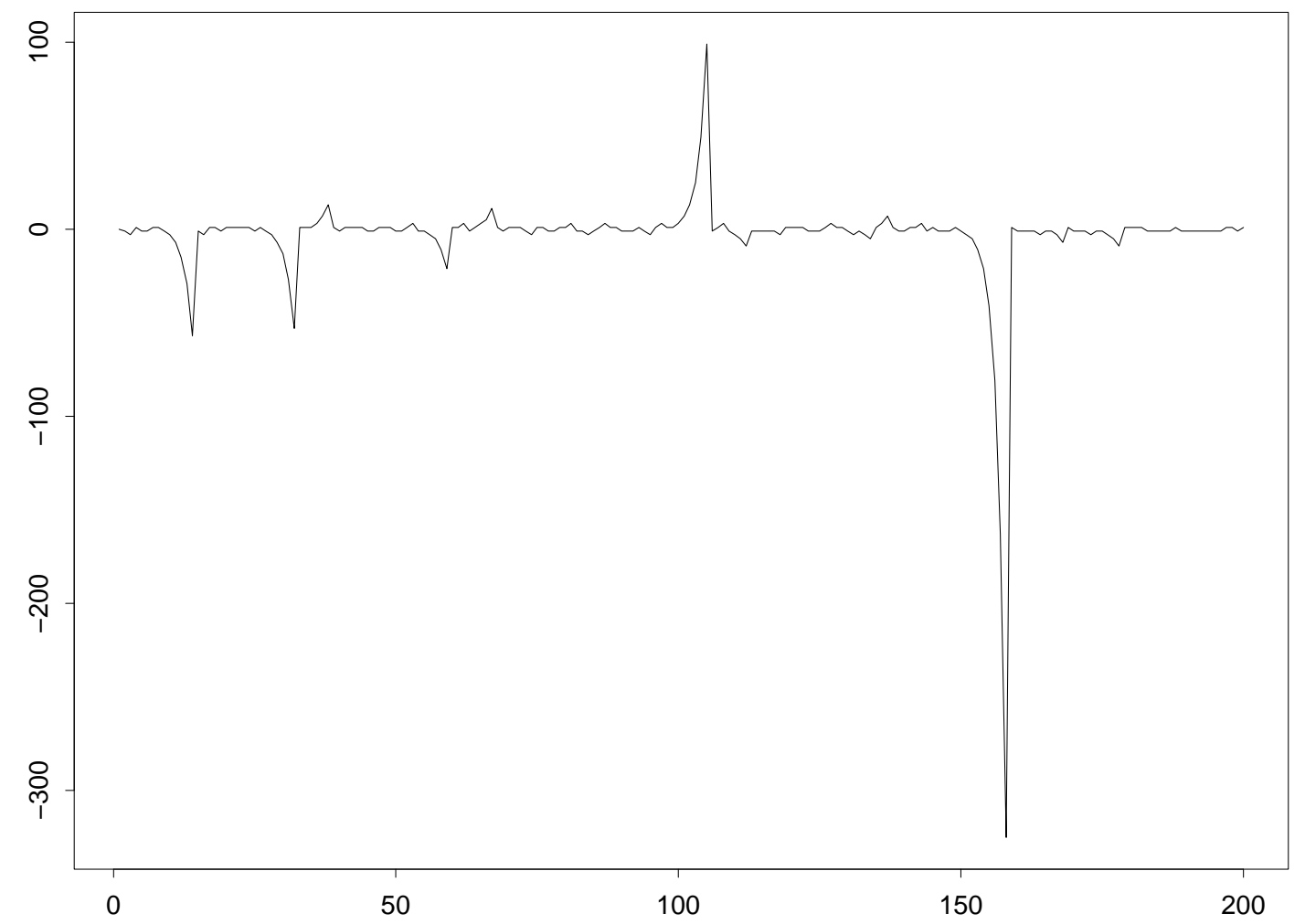

Figure 3: Path of the Stationary Martingale Tree 


\title{
STATIONARY BUBBLE EQUILIBRIA IN RATIONAL EXPECTATION MODELS
}

\author{
C. Gourieroux ${ }^{(1)}$, J. Jasiak ${ }^{(2)}$ and A. Monfort ${ }^{(3)}$
}

October, 23, 2016

The first author gratefully acknowledges financial support of the chair ACPR/Risk Foundation : Regulation and Systemic Risks. We thank S., Auray, I. Ekeland for helpful discussions on the transversality conditions.

\footnotetext{
${ }^{1}$ CREST and University of Toronto.

${ }^{2}$ York University, Canada

${ }^{3}$ CREST.
} 


\section{Stationary Bubble Equilibria in Rational Expectation Models}

Abstract

A linear rational expectation model with current expectations can admit a unique linear stationary dynamic equilibrium for a set of specific parameter values. This paper shows that a multiplicity of stationary dynamic equilibria may arise due to the existence of nonlinear stationary equilibria. These nonlinear equilibria can display bubbles and/or volatility induced mean reversion, consistently with the self-fulfilling prophecies that characterize the rational expectation equilibria. The stationary nonlinear dynamic equilibria require a revised approach in the identification issue, in the impulse response analysis in rational expectation models, or in the test of the present value model that are also discussed in this paper.

Keywords : Rational Expectation, Equilibrium, Stationary Martingale, Speculative Bubble, Volatility Induced Mean-Reversion, Stochastic Economy, Transversality Condition, Identification, Present Value Model. 


\section{Introduction}

Linear dynamic rational expectation (RE) models admit an infinite number of dynamic equilibria that can be either stationary, or nonstationary. So far, the literature has focused on square integrable stationary (SIS) solutions and considered only specific nonstationary equilibria. It has been established that i) the RE model admits a unique SIS equilibrium only for some specific parameter values, while in general, there is an infinite number of SIS equilibria; ii) some nonstationary equilibrium paths feature explosive bubbles, usually explained by the self-fulfilling prophecies inherent to RE models.

This paper shows that, if the finite variance constraint on stationary solutions is relaxed, then there exists an infinite number of real valued or nonnegative stationary solutions to any linear rational expectation model. Note that the stationary solutions are in an uncertain environment the analogues of the constant or steady state solutions in deterministic framework. These stationary solutions with infinite variance include recurrent speculative bubbles and/or volatility induced mean-reversion. Their existence requires a new approach to the identification issue and to the impulse response analysis in linear RE models.

The paper is organized as follows. Section 2 reviews the literature on the set of equilibria in the RE model:

$$
y_{t}=a E_{t} y_{t+1}+z_{t},
$$

where $z_{t}$ represents the exogenous shocks, $E_{t}$ denotes the expectation conditional on the information available at date $t$ and $a$ is a scalar parameter. Section 2 also explains why the condition of finite variance is crucial for obtaining a unique stationary equilibrium when $|a|<1$. Section 3 provides the stationary solutions of the RE model with infinite variance. Two types of stationary nonlinear dynamics are discussed, one of which includes explosive bubbles and the other volatility induced mean-reversion. We also discuss the multiplicity when the solutions are constrained to be nonnegative. Section 4 discusses the identification of the stationary equilibrium and introduces a new approach to the impulse response analysis. Section 5 concludes. The Appendix provides a brief review of the dynamic bubble models considered in the literature and discusses the necessity of transversality conditions. 


\section{Set of Solutions}

This section describes the models and the solutions proposed in the existing economic literature.

\subsection{A rational expectation model}

For expository purpose, let us consider a simple textbook univariate linear rational expectation price model ${ }^{4}$ [see, Taylor (1977), Diba, Grossmann (1988), Flood, Hodrick (1990), Evans (1991)]:

$$
y_{t}=a E_{t}\left(y_{t+1}\right)+z_{t} .
$$

This model follows from an equilibrium model such as :

$$
\begin{cases}q_{t}=\alpha_{1} y_{t}+\alpha_{2} E_{t} y_{t+1}+z_{1 t}, & \text { (demand equation) } \\ q_{t}=\beta y_{t}+z_{2 t}, & \text { (supply equation) }\end{cases}
$$

where $q_{t}$ (resp. $y_{t}$ ) denotes the equilibrium quantity (resp. the equilibrium price), and $z_{1 t}, z_{2 t}$ represent the demand and supply shocks, respectively.

We assume that the joint process $\left(z_{1 t}, z_{2 t}\right)$ is strongly stationary and has a VARMA representation :

$$
\Phi(L)\left(\begin{array}{l}
z_{1 t} \\
z_{2 t}
\end{array}\right)=\Theta(L)\left(\begin{array}{l}
\varepsilon_{1 t} \\
\varepsilon_{2 t}
\end{array}\right),
$$

where $L$ denotes the lag operator, $\left(\varepsilon_{1 t}, \varepsilon_{2 t}\right)$ is a sequence of i.i.d. variables with finite variance and independent components, and the autoregressive polynomial is such that the roots of $\operatorname{det} \Phi(z)=0$ are outside the unit circle. The assumptions of normality and zero mean on $\epsilon_{t}$ are not required.

The equilibrium system (2.2) leads to model (2.1) with parameters:

$$
a=\frac{\alpha_{2}}{\beta-\alpha_{1}}, z_{t}=\frac{z_{1 t}-z_{2 t}}{\beta-\alpha_{1}} .
$$

\footnotetext{
${ }^{4}$ The results are easily extended to models with time-varying discount rate, multivariate models, models with more than one lag, and to expectations at different horizons [see e.g. Gourieroux, Laffont, Monfort (1982), Broze, Gourieroux, Szafarz (1995) for the description of the set of solutions in more general frameworks].
} 
As the equilibrium equation is obtained by matching the demand and supply, the information set contains the current and lagged values of the exogenous components and of the prices :

$$
I_{t}=\left(\underline{z_{1 t}}, \underline{z_{2 t}}\right)=\left(\underline{y_{t}}, \underline{z_{1 t}}, \underline{z_{2 t}}\right),
$$

where $\underline{y_{t}}=\left(y_{t}, y_{t-1}, y_{t-2}, \ldots\right)$, or equivalently $I_{t}=\left(\underline{\varepsilon_{1 t}}, \underline{\varepsilon_{2 t}}\right)=\left(\underline{y_{t}}, \underline{\varepsilon_{1 t}}, \underline{\varepsilon_{2 t}}\right)$.

This model provides also a rational expectation model for the equilibrium quantity:

$$
q_{t}=a E_{t}\left(q_{t+1}\right)+z_{2 t}+\beta z_{t}-a E_{t}\left(z_{2, t+1}\right),
$$

with the same coefficient $a$.

In this rational expectation model, both price and quantity variables take positive values. In a stochastic model, like this one, the equilibrium is an equilibrium of beliefs, that is an equilibrium of the conditional distributions of $y_{t+1}$ given $I_{t}$. In this respect, the outcome of the above model differs from that of a deterministic dynamic model, where the equilibrium is an equilibrium of prices and quantities. As the beliefs are subject to a few linear restrictions, a multiplicity of equilibria arises, due to the so-called selfconfirming belief (or self-fulfilling prophecy) [see e.g. Taylor (1977), Diba, Grossman (1988)a,b and Appendix 1].

\subsection{The linear SIS solutions}

The set of square integrable stationary (SIS) equilibrium prices depends on coefficient $a$ [see e.g. Blanchard (1978), Gourieroux, Laffont, Monfort (1982), Evans, Honkapohia (1986)]. If $|a|<1$, there is a unique SIS solution which

is defined as a linear moving average with an intercept: $y_{t}^{F} \equiv \sum_{j=0}^{\infty} A_{j} \varepsilon_{t-j}+b$. The process $y_{t}^{F}$ is called the forward solution, as for $|a|<1$, it can be obtained from the forward recursion:

$$
y_{t}^{F}=\sum_{h=0}^{\infty} a^{h} E_{t}\left(z_{t+h}\right) .
$$

If $|a|>1$, there is an infinite number of linear SIS solutions. They include the convex combinations of a solution with the same ARMA representation 
as $y_{t}^{F}{ }^{5}$ and of the perfect foresight (or backward) solution :

$$
y_{t}^{1}=a y_{t+1}^{1}+\varepsilon_{t} \Longleftrightarrow y_{t}^{1}=\frac{L}{L-a} \varepsilon_{t} .
$$

In model (2.1), the equilibrium solution has to be positive. For instance if $|a|<1$, the forward solution is admissible iff $\sum_{h=0}^{\infty} a^{h} E_{t} z_{t+h}>0$. This condition requires restrictions on parameter $a$ and on the dynamics of the exogenous variables. For example, the positivity of the forward solution is satisfied if $a>0, z_{t}>0$.

\subsection{The set of all solutions}

Gouriéroux, Laffont and Monfort (1982) have described the set of solutions of the RE model (2.1). These solutions can be written as:

$$
y_{t}=y_{t}^{F}+a^{-t} M_{t}
$$

where $M_{t}$ is a martingale satisfying : $M_{t}=E_{t} M_{t+1}$. Loosely speaking, process $\left(y_{t}\right)$ has two components that are the stationary process $\left(y_{t}^{F}\right)$ with linear dynamics and the martingale-based process $X_{t}=a^{-t} M_{t}$, which can feature nonlinear dynamics. Process $\left(X_{t}\right)$ satisfies $E_{t} X_{t+1}=a^{-1} X_{t}$ and is a submartingale, if $|a|<1$.

\subsection{Fundamental value and rational bubble}

Rational expectations also appear in asset pricing models. In that framework, model (2.1) is $y_{t}=\left(1+r_{f}\right)^{-1} E_{t} y_{t+1}+z_{t}$, where $y_{t}$ is the value of the asset, ${ }^{6}$ which can be "resource, land, painting, jewel (for their consumption value)", or a "technology with decreasing returns to scale" [Tirole (1985)], $z_{t}$ the real rent (dividend), $r_{f}$ the riskfree rate assumed constant and where the information set includes various explanatory factors $z_{1 t}, z_{2 t}$ of the dividends.

\footnotetext{
${ }^{5}$ However this specific solution does no longer admit a forward interpretation, even if we still use the same notation $y_{t}^{F}$ [see e.g. Gourieroux, Laffont, Monfort (1982)].

${ }^{6}$ Model $(2.1)$ can also be seen as a Lucas-type model [Lucas (1978)] : $u^{\prime}\left(c_{t}\right) y_{t}=(1+$ $\left.r_{f}\right)^{-1} E_{t}\left[u^{\prime}\left(c_{t+1}\right)\left(y_{t+1}+d_{t+1}\right)\right]$. Then, model $(2.1)$ is valid with the product of value and marginal utility of consumption as the variable of interest, or to the value itself in the limiting case of a constant marginal utility (risk-neutral investor) (see subsection 3.3.i) and Appendix 3 for a deeper discussion of rational expectation models derived from models with intertemporal optimization).
} 
In such a case, $a=1 /\left(1+r_{f}\right)<1$, and the stationary linear solution is such that : $y_{t}^{F}=\sum_{h=0}^{\infty} \frac{1}{\left(1+r_{f}\right)^{h}} E_{t}\left(z_{t+h}\right)$. The corresponding $y_{t}^{F}$ is equal to the discounted sum of expected future dividends, and is called the fundamental value, or the present value [Shiller (1978), Flood and Garber (1980), Diba, Grossman (1988)], whereas the difference between the current value and the fundamental (present) value tautologically defines the rational bubble, or simply the bubble ${ }^{7}$. However, this interpretation of solution $y_{t}^{F}$ is no longer valid in the general equilibrium model (2.2), especially when $|a|>1$.

\subsection{Real-valued or nonnegative equilibria}

The dynamic RE model (2.1) with $|a|<1$ is a reduced form, which can be derived from a variety of structural models, such as the supply-demand equilibrium, an asset pricing model, a model of the debt-to-GDP ratio, or a first-order condition (Euler condition) to a life-cycle consumption model [Hall (1978)]. The underlying structural model determines the interpretation of the process $\left(y_{t}\right)$ and the information available at date $t$. For example, in the life cycle consumption model, $y_{t}$ is the marginal utility of consumption, $z_{t}=0$, and the information set includes the current and past observations on consumption, earning and asset variables except human capital.

Depending on the structural model, the equilibrium solution $\left(y_{t}\right)$ can be constrained to be nonnegative, or left unconstrained and taking real (positive or negative) values. For example, real-valued equilibria arise in the following applications:

i) In the asset pricing model of Section 2.4, where $y_{t}$ represents the value of a firm defined as the asset less liability, under an assumption that a firm is not automatically defaulted if the asset is less than the liability.

ii) When $z_{t}=0$, dynamic model (2.1) can also be applied to a selffinanced portfolio value under the risk-neutral probability. Without short-sell restrictions, this portfolio can take positive as well as negative values.

iii) Another example concerns the analysis of the balance of payments of different countries in RE models.

\footnotetext{
${ }^{7}$ It is also called the speculative bubble by other authors [see,e.g. Blanchard (1979), Blanchard, Fisher (1989), p. 218-221.] In our paper, we call "speculative" a bubble with explosive trajectory that ends with a burst.
} 
iv) In monetary models of speculative hyperinflation, there is a joint equilibrium on two markets: a consumption good market and a money market [see e.g. Brock (1975), Obstfeld, Rogoff (1983)]. This leads to three conditions on price level, real consumption and nominal money balance, respectively. Under simplifying assumptions, such as a constant consumption profile, two rational expectation equations are obtained, one for the path of the positive price level and another one for the real-valued money balance. In such a framework, one expects that the assumption of endogenous money leads to financial instability, bubbles and extreme volatility. Flat money in an overlapping generation model is probably the most famous example of bubble. The present value of flat money is zero; yet it has a positive price.

v) RE models are often specified in terms of changes of positive variables, without explicit references to any intertemporal optimization. The reason is to render the nominal variables more stationary. The changes can be of any sign. Similarly, the RE models can be directly written in log-transformed variables to eliminate the positivity restrictions [see, e.g. the so-called "adhoc" models in Sargent, Wallace (1975), the "generic canonical form" in Sims (2000), or the Cagan's model in Flood, Garber (1980)]. The RE models in logarithms of variables are often derived by log-linearization of a Dynamic Stochastic General Equilibrium (DSGE).

\section{$3 \quad$ Stationary Solutions with Infinite Variance}

The dynamic rational expectation equilibrium model (2.1) can have multiple stationary solutions even if $|a|<1$, when the stationary equilibrium price is not restricted to be square integrable. The notion of stationarity considered in this paper is the strong stationarity. Process $\left(y_{t}\right)$ is said to be strongly stationary iff the distribution of $\left(y_{t}, y_{t+1}, \ldots, y_{t+k}\right)$ is independent of $t$ for any $k \geq 0$. This notion does not require the existence of the first- and secondorder moments. The additional solutions include martingales which are nonintegrable, and therefore non-square-integrable too. Indeed, as shown below, the conditional expectation $E_{t} M_{t+1}$ may exist even when the unconditional expectation does not.

This section describes the stationary submartingales and explains why the RE models always have an infinite number of stationary solutions when these solutions are not constrained to be positive. That set of solutions is reduced under the positivity restriction, but is still infinite. 


\subsection{Stationary real-valued submartingales}

An integrable stationary submartingale is equal to zero almost surely. To show that, let us denote by $\left(X_{t}\right)$ the submartingale such that:

$$
E_{t}\left(X_{t+1}\right)=(1 / a) X_{t}, \text { with }|a|<1
$$

It follows that :

$$
E_{t}\left(\left|X_{t+1}\right|\right) \geq\left|E_{t}\left(X_{t+1}\right)\right|=(1 /|a|)\left|X_{t}\right|,
$$

and, by taking the expectation of both sides of the inequality :

$$
E\left|X_{t+1}\right| \geq(1 /|a|) E\left|X_{t}\right|
$$

Since $E\left|X_{t}\right|=E\left|X_{t+1}\right|$ by stationarity and $|a|<1$, this inequality implies $E\left|X_{t}\right|=0$, that is $X_{t}=0$ almost surely.

Equivalently, any nonzero stationary submartingale is necessarily nonintegrable (and has infinite variance). As mentioned above, when $|a|<1$, the process $X_{t}=a^{-t} M_{t}$ in (2.3) is a submartingale. Therefore, if the condition of stationarity of the solution is imposed, then $X_{t}$ (and $M_{t}$ ) is non-integrable. However we have at any horizon $h$ :

$$
E_{t}\left(X_{t+h}\right)=\frac{X_{t}}{a^{h}}
$$

Thus, if $X_{t} \neq 0$, the conditional expectations are explosive and no limit for $E_{t}\left(X_{t+h}\right)$ exists, when $h$ tends to infinity, but the conditional distribution of $X_{t+h}$ at date $t$ has a limit for stationary $\left(X_{t}\right)$. Below, we provide four examples of stationary ${ }^{8}$ non-integrable submartingales.

\subsubsection{Stable noncausal process}

A noncausal stable $\mathrm{AR}(1)$ is a strongly stationary process that satisfies the noncausal (or forward) autoregression [see Gourieroux, Zakoian (2016)]:

$$
y_{t}^{*}=\rho y_{t+1}^{*}+\varepsilon_{t}^{*}, 0 \leq \rho<1,
$$

\footnotetext{
${ }^{8}$ More precisely, processes $\left(y_{t}^{F}\right),\left(X_{t}\right)$ have to be jointly stationary.
} 
where the $\varepsilon_{t}^{*}$ are i.i.d. variables with a stable distribution ${ }^{9}$ with stability index $s, 0<s \leq 1$. This process is Markov in both the calendar and reverse times and admits a causal nonlinear autoregressive representation [see e.g. Rosenblatt (2000)] :

$$
y_{t}^{*}=g\left(y_{t-1}^{*}, \eta_{t} ; s, \rho\right),
$$

where $\left(\eta_{t}\right)$ is a strong Gaussian white noise, independent of the lagged values of $\left(y_{t}^{*}\right)$. The noise $\eta_{t}$ is an increasing function of $y_{t}^{*}$ for a given $y_{t-1}^{*}$, and is the Gaussian nonlinear innovation of Markov process $\left(y_{t}^{*}\right)$. Indeed, let us denote by $F\left(y_{t}^{*} \mid y_{t-1}^{*}\right)$ the conditional cumulative distribution function (c.d.f.) of $y_{t}^{*}$ given $y_{t-1}^{*}$. If function $F\left(. \mid y_{t-1}^{*}\right)$ is invertible, we know that $u_{t}=F\left(y_{t}^{*} \mid y_{t-1}^{*}\right)$ follows a uniform distribution on $[0,1]$. Thus $\eta_{t}=\Phi^{-1}\left(u_{t}\right)=$ $\Phi^{-1}\left[F\left(Y_{t}^{*} \mid y_{t-1}^{*}\right)\right]$, where $\Phi$ is the c.d.f. of the standard normal, follows $N(0,1)$, conditional on $y_{t-1}^{*}$. As $\left(y_{t}^{*}\right)$ is a Markov process, $\eta_{t}$ is also standard normal, conditional on $y_{t-1}^{*}=\underline{\eta_{t-1}}$. This implies the independence between $\eta_{t}$ and $\eta_{t-1}$, and the fact that $\overline{\left(\eta_{t}\right)}$ is a Gaussian white noise. The nonlinear autoregression (3.2) is obtained by inverting the relation that defines $\eta_{t}$.

As the reverse time innovation $\varepsilon_{t}^{*}$ has fat tails, $y_{t}^{*}$ does not have finite first and second-order unconditional moments. Its first and second-order moments conditional on the future are infinite as well. Nevertheless, it is shown in Gourieroux, Zakoian (2016) that this process has a first-order conditional moment given the past:

$$
E\left(y_{t+1}^{*} \mid \underline{y_{t}^{*}}\right)=\rho^{(s-1)} y_{t}^{*},
$$

where the autoregressive coefficient $\rho^{(s-1)}$ is larger than 1 . Thus this process is a stationary submartingale when $s<1$, a stationary martingale if $s=1$.

The path of a noncausal autoregressive stable process features local explosions followed by crashes. This is illustrated in Figure 1 by the simulated paths of noncausal autoregressive Cauchy processes with $\rho=0.5$ and $\rho=0.8$, respectively, and a Cauchy error $\varepsilon_{t}^{*}$. This corresponds to the limiting case of a martingale with $s=1$ for the stability index (and $a=1$ in the associated RE model).

[Insert Figure 1 : Path of Noncausal Stable Process]

\footnotetext{
${ }^{9} \mathrm{~A}$ stable distribution is a continuous distribution with characteristic function : $E[\exp (i u \varepsilon)]=\exp \left(-c|u|^{s}\right)$, where $c, c>0$, is a scale parameter. When $s=1$, we get the Cauchy distribution, when $s=1 / 2$ the Levy distribution.
} 
The positive or negative explosions cannot last indefinitely, as they are always followed by a crash. Whereas the bubble explodes in expectation, it is collapsing systematically in realization. ${ }^{10}$. When the above model is applied to the pricing of a monetary unit, the role of the money becomes a pure storage value [Samuelson (1958)]: "Temporarily money can yield a rate of interest lower than the market rate because it is used for transactions. But this effect must disappear sufficiently fast to allow a bubble" [Tirole (1985), p.1517]. Note that the simulated path shows that this process is compatible with "the reality where bubbles seem to deflate over several weeks or even months" [see the conclusion in Brunnermeier (2008)].

\subsubsection{Time discretized diffusion process}

Let us now consider the diffusion process defined by :

$$
d y_{t}^{*}=\delta y_{t}^{*} d t+\sigma\left(y_{t}^{*}\right) d W_{t},
$$

where $\left(W_{t}\right)$ is a Brownian motion, $\sigma($.$) the volatility function and \delta$ a positive scalar. Since $\delta$ is positive, this diffusion process has no (drift induced) mean reversion. This process satisfies the stationarity condition (more precisely the positive recurrence condition that implies ergodicity), if and only if, the two following conditions are satisfied [see Durett (1996), p221, or Kutoyants (2004), Th. 1.16] :

$$
\int_{0}^{x} \exp \left\{-2 \delta \int_{0}^{y} \frac{u}{\sigma^{2}(u)}\right\} d y \rightarrow \pm \infty, \text { as } x \rightarrow \pm \infty
$$

and

$$
\int_{-\infty}^{+\infty} \frac{1}{\sigma^{2}(y)} \exp \left(2 \delta \int_{0}^{y} \frac{u}{\sigma^{2}(u)} d u\right) d y<\infty
$$

\footnotetext{
${ }^{10}$ This example contradicts some largely diffused assertions, such as "The bubble process obeys the martingale equation...Clearly, the bubble component, if it exists, can never burst" [Montrucchio, Privileggi (2001) p. 165, and the discussion in Diba, Grossman (1988) b, Section III], or as "the bubble component will dominate the fundamental component" [Evans (1991), p.923]. This also question the notion of "growth" of the bubble component largely used in the literature. Indeed the sequence of predictions $E\left(y_{t+k}^{*} \mid y_{t}^{*}\right)$ explodes with $h$, at rate $\rho^{(s-1)}$, whereas the local explosions observed on the path of $y_{t}^{*}$ have a rate strictly larger than $\rho^{(s-1)}$, and the sequence of conditional distributions converges..
} 
Let us now assume that these conditions hold and consider the time discretized diffusion, that is the process $\left(y_{t}^{*}\right)$ observed at discrete dates $t=$ $1,2, \ldots$. This process is Markov in discrete time. We have :

$$
E_{t}\left(y_{t+d t}^{*}\right)=(1+\delta d t) y_{t}^{*} \simeq \exp (\delta d t) y_{t}^{*},
$$

and by iterated expectations, for a small $d t$ and a large number of iterations : $E_{t}\left(y_{t+1}^{*}\right)=(\exp \delta) y_{t}^{*}$. Thus, for $\delta$ such that $\exp \delta=1 / a$ with $0<a<1$, the process becomes a submartingale which can be added to the forward solution in order to obtain an additional new stationary solution to RE model (2.1), whenever the nonlinear innovation of $\left(y_{t}^{*}\right)$ is a function of $\left(\varepsilon_{1 t}, \varepsilon_{2 t}\right)$.

The path of the time discretized diffusion displays endogenous regimes of extremes, before reverting to more frequent values. In the literature, this pattern is called the volatility induced mean-reversion [see Conley et al. (1977)].

[Insert Figure 2: Path of a Process with Volatility Induced Mean-Reversion]

This is illustrated in Figure 2 by the simulated paths of time discretized diffusion processes with $\delta=0, \sigma\left(y^{*}\right)=\sqrt{1+\left|y^{*}\right|}$ and $\sigma\left(y^{*}\right)=\sqrt{1+y^{* 2}}$, respectively, which correspond to the limiting case of a martingale (i.e. to $a=1$ in the associated RE model). When the process takes extreme values, the volatility becomes large. As a consequence, the process either remains extreme-valued in the future, or reverts to its frequently observed values. In general, clusters of extreme values are observed prior to the "mean reversion". Volatility induced mean reverting submartingales can also be constructed from autoregressive processes with ARCH effects. The basic model of this type is the double autoregressive model [see Borkovec, Kluppelberg (2001), Ling (2007), and Nielsen, Rahbek (2014) for an extension to multivariate processes.]

\subsubsection{A Stationary Martingale Tree}

The examples of stationary (sub)martingales given before may be difficult to interpret, especially for financial practitioners who are more familiar with the binomial trees a la Cox, Ross, Rubinstein (1979).

This subsection provides an example of a stationary submartingale tree. This tree features both speculative bubbles and volatility induced mean re- 
version. It also has deterministic dynamics in reverse time during the "explosive" bubble spell.

The process is defined as follows:

$$
y_{t+1}= \begin{cases}2 y_{t}+1, & \text { with probability } 1 / 4 \\ 2 y_{t}-1, & \text { with probability } 1 / 4 \\ +1, & \text { with probability } 1 / 4 \\ -1, & \text { with probability } 1 / 4\end{cases}
$$

where the regimes in each period are drawn independently. In some sense, the regime indicator plays the role of a shock at each time $t$.

For a given value $y_{t}$, we have:

$$
E\left(y_{t+1} \mid y_{t}=y\right)=\frac{1}{4}\left(2 y_{t}+1+2 y_{t}-1+1-1\right)=y_{t},
$$

which is the martingale condition. A stationary submartingale tree is obtained by replacing the scale factor 2 in the first step of the binomial tree by a larger scale factor.

This model can be interpreted as follows:

i) It can be considered as a binomial tree, which is observed every two periods. In the first period, we have:

$$
\tilde{y}_{t+1}= \begin{cases}2 y_{t}, & \text { with probability } 1 / 2, \\ 0, & \text { with probability } 1 / 2 .\end{cases}
$$

In the second period we get:

$$
y_{t+1}=\tilde{y}_{t+1}+\epsilon_{t+1},
$$

where $\epsilon_{t+1}$ takes values $+1,-1$, with equal probability $1 / 2$, and is independent of the regime indicator of the first period. Thus, the movement in the first period creates a potential explosion, or a mean reversion, whereas the movement in the second period is the standard dynamics found in the Cox, Ross, Rubinstein and Black, Scholes models.

ii) It can also be considered as an alternative to the bubble model proposed by Blanchard, Watson (1982) (see Appendix 2 ii)).

By construction the process can take odd values only, except for the first value. It regularly returns to +1 and -1 , due to the two last regimes in (3.7) (a recurrence property of this Markov chain). It can also display explosive trajectories in the set of values larger than 3 (resp. smaller than -3) during a 
period of "explosion". In order to describe this phenomenon, let us consider an initial value $y=3$ and all possible trajectories for the first two regimes in (3.7) (The results are similar for $y=-3$, say, and lead to a negative bubble).

At step $n, n=0,1,2, \ldots$, the odd numbers $2^{n+1}+1+2 m, m=0,1, \ldots, 2^{n}-$ 1 can be reached. All these trajectories are explosive. Their rate of explosion is stochastic, with an additive increase between $2^{n}$ and $2^{n+1}$ at each step $n$.

It is interesting to examine the martingale tree in reverse time. During an explosive bubble spell (either positive, or negative), the process is deterministic in reverse time while it is stochastic in calendar time. For instance, if the current observed value is $y=251$, say, the previous values are necessarily $125,63,31,15,7,3$. If the current observed value is 1233 , say, the previous values in calendar time are necessarily $617,309,155,77,39,19,9,5,3$.

The martingale tree is a Markov process, which is recurrent and stationary. Its stationary distribution is easily derived by using the property of deterministic bubble trajectories in reverse time.

First, we compute the marginal probabilities of +1 and -1 . We have:

$$
\begin{aligned}
P\left(y_{t+1}=1\right) & =P\left(y_{t+1}=1 \mid y_{t}=1\right) P\left(y_{t}=1\right)+P\left(y_{t+1}=1 \mid y_{t} \neq 1\right)\left[1-P\left(y_{t}=1\right)\right] \\
& =\frac{1}{2} P\left(y_{t}=1\right)+\frac{1}{4}\left[1-P\left(y_{t}=1\right)\right]=\frac{1}{4}+\frac{1}{4} P\left(y_{t}=1\right) .
\end{aligned}
$$

It follows that the stationary probability of 1 is:

$$
P\left(y_{t}=1\right)=1 / 3
$$

and by symmetry:

$$
P\left(y_{t}=-1\right)=1 / 3 \text {. }
$$

Next, we find the marginal probabilities of values in a positive bubble. We have:

$$
\begin{aligned}
P\left(y_{t+1}=3\right) & =\frac{1}{4} P\left(y_{t}=1\right)=\frac{1}{3} \frac{1}{4}, \\
P\left(y_{t+1}=5\right) & =\frac{1}{4} P\left(y_{t}=3\right)=\frac{1}{3}\left(\frac{1}{4}\right)^{2}, \ldots
\end{aligned}
$$

and so on. At step $n$ of the explosive bubble with positive values, we have:

$$
P\left(y_{t}=2^{n+1}+1+2 m\right)=\frac{1}{3} \frac{1}{4^{n+1}}
$$


It is easy to check that these probabilities sum up to 1 . Indeed the probabilities of positive values sum up to 0.5 as shown below:

$$
\begin{aligned}
P\left(y_{t}=1\right)+\sum_{n=0}^{\infty} \sum_{m=0}^{2^{n}-1} P\left(y_{t}=2^{n+1}+1+2 m\right) & =\frac{1}{3}+\sum_{n=0}^{\infty} \frac{1}{3} \sum_{m=0}^{2^{n}-1}\left(\frac{1}{4^{n+1}}\right) \\
& =\frac{1}{3}\left[1+\sum_{n=0}^{\infty}\left(\frac{2^{n}}{4^{n+1}}\right)\right] \\
& =\frac{1}{3}\left[1+\frac{1}{4} \sum_{n=0}^{\infty} \frac{1}{2^{n}}\right] \\
& =\frac{1}{3}\left(1+\frac{1}{4} \frac{1}{1-1 / 2}\right)=\frac{1}{3}\left(1+\frac{1}{2}\right)=\frac{1}{2} .
\end{aligned}
$$

In a similar way one can show that the probabilities of negative values sum up to 0.5 as well.

It is easy to check that the process features volatility induced mean reversion. Indeed, the change in the process is:

$$
\Delta y_{t+1}= \begin{cases}y_{t}+1, & \text { with probability } 1 / 4 \\ y_{t}-1, & \text { with probability } 1 / 4 \\ -y_{t}+1, & \text { with probability } 1 / 4 \\ -y_{t}-1, & \text { with probability } 1 / 4\end{cases}
$$

and the conditional variance is:

$$
V\left(y_{t+1} \mid y_{t}\right)=E\left[\left(\Delta y_{t+1}\right)^{2} \mid y_{t}\right]=\frac{1}{2}\left(y_{t}+1\right)^{2}+\frac{1}{2}\left(y_{t}-1\right)^{2}=y_{t}^{2}+1
$$

Thus, the larger $y_{t}^{2}$, the larger the variance. We conclude that this model displays volatility induced mean reversion towards 1 , or -1 .

In this simple framework:

i) We know with certainty if the process is in a phase of a positive (resp. negative) bubble, by checking if $y_{t} \geq 3$ (resp. $y_{t} \leq-3$ ).

ii) We can predict the time to bubble crash. Conditional on $y_{t} \geq 3$, we stay on a bubble with probability $1 / 2$, and we return to either \pm 1 , with probability $1 / 2$. Thus, this stochastic time to crash follows a Pascal distribution with parameter $1 / 2$. In particular, in this basic tree, the distribution of the 
time to crash does not depend on the time already spent in the bubble, contrary to the noncausal $\mathrm{AR}(1)$ Cauchy process, and the average sejourn time in a bubble is 2 .

As an illustration, Figure 3 shows a trajectory of such a process.

[Insert Figure 3: Path of the Stationary Martingale Tree]

\subsubsection{A Switching Regime Autoregression}

Let us consider two independent white noises $\left(z_{t}\right),\left(\varepsilon_{t}\right)$, where the $z_{t}^{\prime} s$ follow Bernoulli distributions $\mathcal{B}(1, p)$, and the $\varepsilon_{t}^{\prime} s$ are Gaussian with zero-mean and variance $\sigma^{2}$. The autoregressive model is defined by :

$$
y_{t}=\alpha \frac{z_{t}}{p} y_{t-1}+\varepsilon_{t}, \alpha>1, p<1,
$$

or equivalently at time $t, y_{t}$ is drawn in the distribution $N\left(\alpha y_{t-1}, \sigma^{2}\right)$ with probability $p$, and in the distribution $N\left(0, \sigma^{2}\right)$ with probability $1-p$. This autoregressive dynamics admits a stationary solution with the nonlinear moving average representation :

$$
y_{t}=\varepsilon_{t}+\frac{\alpha}{p} z_{t} \varepsilon_{t-1}+\left(\frac{\alpha}{p}\right)^{2} z_{t} z_{t-1} \varepsilon_{t-2}+\ldots
$$

Let us introduce the first time $\tau$ such that $z_{t-\tau}=0$. We have $P[\tau=$ $h]=(1-p) p^{h-1}, h \geq 1$. Moreover, when $\tau=h$, the variable $y_{t}$ is equal to

$y_{t}=\varepsilon_{t}+\frac{\alpha}{p} \varepsilon_{t-1}+\ldots+\left(\frac{\alpha}{p}\right)^{h-1} \varepsilon_{t-h+1}$. We deduce the stationary distribution of $y_{t}$ as the mixture of Gaussian distributions $N\left(0, \sigma^{2} \frac{1-(\alpha / p)^{2 h}}{1-(\alpha / p)^{2}}\right)$, with weights $(1-p) p^{h-1}$.

The conditional expectation of $y_{t}$ given $y_{t-1}$ is : $E\left(y_{t} \mid y_{t-1}\right)=\alpha y_{t-1}$, but the process has marginal fat tails. For instance, its unconditional variance is equal to :

$$
V\left(y_{t}\right)=\frac{\sigma^{2}(1-p)}{1-(\alpha / p)^{2}} \sum_{h=1}^{\infty} p^{h-1}\left[1-(\alpha / p)^{2 h}\right]=+\infty,
$$

since the general term of the series is of order $\left(\alpha^{2 h} / p^{h}\right)$, with $\alpha>1,1 / p>1$. 
The first-order moment $E\left(y_{t}\right)$ is also infinite, but the direct proof demands more computations.

Note that the probability of the regime does not depend on the current state $y_{t-1}$ to ensures the submartingale property. In this respect this switching regime is not a special case of STAR process [see Tong (1990), Bec et al. (2010)].

\subsection{Stationary nonnegative submartingales}

The stationary submartingales considered in Section 3.1 can take positive as well as negative values. Let us now focus on stationary submartingales constrained to take nonnegative values only. Examples of stationary nonnegative submartingales will be defined in an indirect way in Section 3.3. ii).

Lemma 1: For a stationary nonnegative martingale $\left(M_{t}\right)$, there exists a variable $M_{\infty}$ taking non-negative values and possibly the value $+\infty$, such that $M_{t}$ converges a.s. to $M_{\infty}$.

\section{Proof:}

Let us consider the process $X_{t}=\exp \left(-M_{t}\right)-1$. This process takes values between -1 and 0 , is uniformly integrable and such that:

$$
E_{t}\left(X_{t+1}\right)=E_{t}\left[\exp \left(-M_{t+1}\right)\right]-1 \geq \exp \left(-E_{t} M_{t+1}\right)-1=X_{t},
$$

by Jensen (convexity) inequality.

Next, we can apply Doob's martingale convergence theorem [see e.g. Williams (1991)]. There exists a variable $X_{\infty}$ such that $X_{t}$ converges a.s. and in $L^{1}$ to $X_{\infty}$. The result follows because the a.s. convergence also applies to the sequence $M_{t}$ with $M_{\infty}=-\log \left(1+X_{\infty}\right)$ Q.E.D.

The limit variable $X_{\infty}$ may take the values 0 and -1 with strictly positive probabilities. Then, $M_{\infty}$ may take the values 0 and $+\infty$.

Lemma 1 implies the following result :

Proposition 1: A nonnegative stationary solution to the equation $y_{t}=$ $a E_{t} y_{t+1}$, with $0<a<1$ is such that the limit of the associated martingale is $M_{\infty}=0$.

Proof: $M_{t}=a^{t} y_{t}$ is a nonnegative martingale. Therefore, it follows that $a^{t} y_{t} \rightarrow M_{\infty}$ a.s.. Since process $\left(y_{t}\right)$ is stationary, the marginal distribution 
of $\left(y_{t}\right)$ is equal to the marginal distribution of $\lim M_{\infty} / a^{t}$ (because the a.s. convergence implies the convergence in distribution). That "stationary distribution" is non-degenerate, iff there is no probability mass at $+\infty$, that is, if $M_{\infty}=0$ a.s. The result follows.

QED

\subsection{An infinite set of stationary equilibria}

The results of Sections 3.1-3.2 can be used to derive the set of stationary equilibria of RE model (2.1). We distinguish the case of real-valued and nonnegative solutions. We also discuss the common practice of log-linearization of DSGE models.

\section{i) Real-valued equilibria}

Let us return to the rational expectation model (2.1) with $0<a<1$, and introduce a standard Gaussian white noise $\eta_{t}$, which is a function of structural shocks $\varepsilon_{1 t}, \varepsilon_{2 t}^{11}$. The stationary submartingales described in Section 3.1 can be used to construct an enlarged set of stationary solutions of the RE model (2.1) and more generally of any RE model. For expository purpose, let us consider the noncausal stable AR(1) process defined in Section 3.1.1 by :

$$
\begin{aligned}
y_{t}^{*}(s, \rho) & =g\left[y_{t-1}^{*}(s, \rho), \eta_{t} ; s, \rho\right] \\
\Leftrightarrow y_{t}^{*}(s, \rho) & =\rho y_{t+1}^{*}(s, \rho)+\varepsilon_{t}^{*}(s) .
\end{aligned}
$$

The process :

$$
y_{t}(s, \rho)=y_{t}^{F}+y_{t}^{*}(s, \rho)
$$

is a stationary solution to dynamic RE model (2.1), whenever :

$$
\rho=a^{1 /(1-s)} .
$$

as $y_{t}^{*}(s, \rho)$ is a submartingale with scale function $\rho^{s-1}=1 / a$, [see (3.3)].

This implies that, even if $0<a<1$, the $\mathbf{R E}$ equilibrium model (2.1) has an infinite number of stationary solutions. As the stability index $s$ can be chosen arbitrarily, by taking linear combinations of such submartingales, any process of the type :

\footnotetext{
${ }^{11}$ Such a standard noise can be obtained by selecting a function of $\varepsilon_{1 t}, \varepsilon_{2 t}: a_{t}=$ $a\left(\varepsilon_{1 t}, \varepsilon_{2 t}\right)$, say. Then $\eta_{t}$ is derived as $\eta_{t}=\Phi^{-1}\left[F_{a}\left[a_{t}\right]\right]=\Phi^{-1}\left[F_{a}\left[a\left(\varepsilon_{1 t}, \varepsilon_{2 t}\right]\right]\right.$, where $F_{a}$ denotes the c.d.f. of $a_{t}$, and $\Phi$ the c.d.f. of the standard normal
} 


$$
y_{t}=y_{t}^{F}+\Sigma_{s} \lambda(s) y_{t}^{*}\left[s, a^{1 /(1-s)}\right],
$$

is also a stationary solution of RE model (2.1). This contradicts Propositions 1 and 2 in Blanchard, Kahn (1980), for example.

The bubbles depend on the innovation of economic variables and have effects on the demand and supply. Thus, they are economically meaningful. These are intrinsic bubbles in the standard terminology [Froot, Obstfeld (1991), Brunnermeier (2008), Gurkaynak (2008)] and do not originate from self-confirming beliefs based on irrelevant variables, commonly called the sunspots (or animal spirits). 12

In practice, when $|a|<1$, it is common to select the "forward" solution as if it were the unique stationary solution of the rational expectation model. This practice disregards all stationary solutions with fat tails and nonlinear dynamics [see e.g. Pesaran, Smith (2011), p7, Leeper et al. (2013), eq. (4) and eq. (17), for recent examples of this practice, and the textbook by Ljunnqvist, Sargent (2000), Section 13.3 that reads: "In the general equilibrium model that we will describe later, the bubble term always equals zero."]

In the literature, the forward solution is often selected because of artificial constraints or approximations, which ensure that the process is integrable, and then are not innocuous. These constraints may be the consequence of a) borrowing constraints ${ }^{13}$ [see e.g. Ljungqvist, Sargent (2000), Chapter 8, 13, 17, for the discussion of such constraints], or of b) a state space discretization leading to a finite state space [see e.g. Ljungqvist, Sargent (2000), Section 4.2 ], or of c) of distributional assumptions on the solution such as the lognormality [see e.g. Blanchard, Weil (2001), Section 4 for such an assumption in a storage economy], or of d) assumptions on the dynamics, such as a deterministic explosion rate [see e.g. Bertocchi (1991), eq. (4)].

\footnotetext{
${ }^{12}$ All these stationary solutions are intrinsic based on the smallest number of structural shocks. This constradicts the idea that bubble solution can be avoided by restricting attention to the "minimal state variable" solutions that depend only on fundamentals [McCallum (1983)].

${ }^{13}$ These artificial restrictions are often introduced to rule out (sustainable) Ponzi schemes. However, they also rule out other dynamics, as shown in the example of volatility induced mean-reverting process, or processes with explosive bubbles with burst. "It is not rare to find in the literature that some mechanism is called irrevelant because it is killed by the artificial constraints of the core. In many instances that can be corroborated by data, such results are really indictments of the artificial constraints, not of the mechanisms" [Caballero (2010)].
} 
Such implicit restrictions are also introduced to facilitate the estimation of parameters by instrumental variable [see e.g. Fuhrer, Oliver (2004), Gali et al. (2001), Dufour, Khalaf, Kichian (2006, (2010) for the so-called identification robust analysis of DSGE models or by Bayesian methods]. For instance let us consider the instrumental variable approach. Typically model (2.1) is rewritten as :

$$
y_{t}=a y_{t+1}+z_{t}+a \eta_{t+1},
$$

where the expectation error $\eta_{t+1}$ is such that $E_{t} \eta_{t+1}=0$, or equivalently parameter $a$ is defined by the conditional moment restriction :

$$
E_{t}\left[y_{t}-a y_{t+1}-z_{t}\right]=0,
$$

assuming for expository purpose that $z_{t}$ is observed. However, the implementation of a standard instrumental variable approach based on instruments $y_{t}$ and/or $z_{t}$, say, assumes the existence of cross moments such as $E\left(y_{t+1} y_{t}\right)$, and also the conditional homoscedasticity of the error term $V_{t} \eta_{t+1}=c t$. These assumptions are satisfied by the stationary integrable solution only, not by the nonlinear stationary solutions. Indeed the latter ones are non integrable and feature volatility effects. Other estimation approaches such as the maximum likelihood method or Bayesian approaches implicitly assume that a specific solution has been selected without justifying this choice [see e.g. Schorfheide (2015), Gallant, Giacomini, Ragusa (2014)].

The stationary solutions can take positive as well as negative values. These solutions are not ruled out by the basic absence of arbitrage opportunity condition. For example, let us consider a self-financed portfolio value $y_{t}$ such that $y_{t}=a E_{t}\left(y_{t+1}\right)$ and an investor selling (resp. buying) that portfolio at time $t$ and buying (resp. selling) it back in the long run. Due to the stationarity of the bubble, we see that:

$$
\lim _{h \rightarrow \infty} P\left(y_{t+h}>y_{t} \mid y_{t}\right)=G\left(y_{t}\right),
$$

where $G$ is the c.d.f. of the stationary distribution of the process. For a noncausal process, a time-discretized diffusion, or the switching regime autoregression, this stationary distribution is continuous on $(-\infty,+\infty)$. Therefore, $G\left(y_{t}\right) \neq 0,1$ and there is no asymptotic arbitrage opportunity. ${ }^{14}$

\footnotetext{
${ }^{14} \mathrm{~A}$ similar result is valid when the selling time is a predetermined finite stopping time.
} 
The absence of arbitrage opportunity is satisfied in this case where the expected future value tends to infinity:

$$
\lim _{h \rightarrow \infty} E_{t}\left(y_{t+h}\right)=\lim _{h \rightarrow \infty} \frac{1}{a^{h}} y_{t}=\operatorname{sng}\left(y_{t}\right) \infty .
$$

This is due to the fact that the concept of arbitrage is not defined in mean-variance terms. Equivalently, the value represented by $y_{t}$ can diverge in mean $\left(L^{1}\right)$, without having an asymptotically explosive realized trajectory.

When model (2.1) is the set of Euler conditions in a Lucas-type economy, the existence of multiple stationary solutions with bubbles can also be explained as follows. As the demand is derived as a solution of an intertemporal optimization problem, it satisfies the Euler's conditions, as well as the transversality conditions. ${ }^{15}$ These latter conditions are often introduced in order to obtain a unique solution, that is to rule out the bubbles [see the discussion in Kamihigashi (2008)]. However, the transversality conditions may be not necessary. In particular, bubbles are not ruled out in a CCAPM model with unbounded utility [see Kamihigashi (1998), (2004) Montrucchio, Privileggi (2004) and Appendix 3].

To summarize, a multiplicity of real-valued stationary solutions to the RE model is obtained even when $|a|<1$ because of stationary (non integrable) submartingales. These stationary submartingales can be combined by choosing:

- the stability index for the noncausal stable submartingale and the dependence of nonlinear innovations on the exogenous shocks;

- the volatility function in the time discretized diffusion and the dependence of nonlinear innovations on the exogenous shocks;

- by introducing appropriate endogenous regimes in quadrinomial trees.

- or by considering different stationary and nonstationary switching regimes.

\footnotetext{
${ }^{15}$ Transversality conditions may appear when the agents are infinitely living, have the same preferences, and share the same information. They do not have to be introduced in overlapping generations models, under asymmetric information, under disagreement over the underlying value, or if there is limited liability [see e.g. Camerer (1989), Hong, Sraer (2013)]. Likely also, if there is a finite, but imprecisely known market depth [see Enders, Hakenes (2014) for a study of this framework].
} 
In Lucas type model these solutions are rational, i.e. they are compatible with agents maximizing their intertemporal utility.

The multiplicity of solutions is specific to stochastic models. Indeed, in the associated deterministic model: $y_{t}=a y_{t+1}+z$, say, all bubbles are nonstationary. They never burst, once they start growing.

\section{ii) Nonnegative equilibria}

As mentioned earlier, the equilibria must be nonnegative when the variable of interest is a price, or the quantity of a physical good. Stationary patterns usually characterize quantities. But price processes, such as commodity prices, exchange rates, or real prices may have stationary patterns too.

The set of stationary equilibria is reduced under the nonnegativity restriction, but remains infinite.

Indeed, let us first consider a RE model with a constant negative $z$ :

$$
y_{t}=a E_{t} y_{t+1}+z, 0<a<1, z<0 .
$$

Note that a model with negative $z$ may be appropriate for the dynamics of a commodity price, where $z$ is interpreted as a convenience yield, i.e. a cost of storage.

Lemma 2 : Model (3.15) has an infinite number of positive stationary solutions.

Proof : i) First note that the forward solution $y_{t}^{0 F}=\frac{z}{1-a}$ is negative and thus is not admissible.

ii) Another solution is the process defined by :

$$
y_{t+1}^{0}=\left\{\begin{array}{l}
\frac{1}{a p} y_{t}^{0}, \text { with probability } p, 0<p<1, \\
-\frac{z}{a(1-p)}, \text { with probability } 1-p,
\end{array}\right.
$$

where the regime indicator is based on the latent innovations ${ }^{16}\left(\varepsilon_{1, t+1}, \varepsilon_{2, t+1}\right)$.

\footnotetext{
${ }^{16}$ There latent innovations may come from the exogenous processes with effects on demand and supply, even if a specific linear combination of these exogenous processes, i.e. $z$, is constant.
} 
This process is positive, such that :

$$
E_{t} y_{t+1}^{0}=\frac{1}{a} y_{t}^{0}-\frac{z}{a}
$$

This process is stationary, with stationary distribution :

$$
P\left[y_{t}=\frac{-z}{a(1-p)} \frac{1}{(a p)^{n}}\right]=(1-p) p^{n}, n=0, \ldots,
$$

and not integrable.

iii) Other real valued solutions are obtained by considering the combinations :

$$
y_{t}(\lambda, z, p)=\lambda y_{t}^{0}+(1-\lambda) y_{t}^{0 F}
$$

Since $y_{t}(\lambda, z, p)=\lambda y_{t}^{0}+(1-\lambda) \frac{z}{1-a}>\frac{-\lambda z}{a(1-p)}+\frac{(1-\lambda) z}{1-a}$,

this combination is a positive solution, when $\lambda>\frac{a(1-p)}{1-a p}$.

To summarize we get an infinite set of positive stationary solutions parametrized by $\lambda$ and $p$.

QED

Let us now consider the model :

$$
y_{t}=a E_{t} y_{t+1}+z_{t}, z_{t} \geq 0,
$$

where the nonnegative process $z_{t}$ is stationary integrable. This corresponds to the model of Section 2.4. For this model the forward solution given by :

$$
y_{t}^{F}=\sum_{h=0}^{\infty} a^{h} E_{t} z_{t+h},
$$

is now positive and then an admissible solution.

Proposition 2 : Model (3.16) has an infinite number of nonnegative stationary solutions.

Proof : Let us denote : 


$$
x_{t}(z, p)=y_{t}(1, z, p)-y_{t}(0, z, p)=y_{t}^{0}-y_{t}^{0 F}, z<0,0<p<1 .
$$

This process is a positive stationary solution of the homogenous REmodel : $x_{t}(z, p)=a E_{t} x_{t+1}(z, p)$.

Therefore $y_{t}^{F}+x_{t}(z, p)$ is a positive stationary solution of model (3.14). This set of solutions is parametrized by $z$ and $p, z<0,0<p<1$.

QED

Let us now discuss consequences of Proposition 2. For expository purpose, we denote by $\left(\varepsilon_{t}\right)$ an i.i.d. process taking values 1 and 3 with probability $p=1 / 2$, choose $z_{t}=1-\varepsilon_{t}$, and use $\left(\varepsilon_{t}-1\right) / 2$ as the indicator function for defining the bubble process $y_{t}^{0}-y_{t}^{0 F}$. Since $E_{t}\left(z_{t+h}\right)=-1$, the forward solution is :

$$
y_{t}^{F}=1-\varepsilon_{t}-\frac{a}{1-a},
$$

whereas a solution with bubble is :

$$
\begin{aligned}
y_{t} & =y_{t}^{F}+y_{t}^{0}-y_{t}^{0 F} \\
& =1-\varepsilon_{t}-\frac{a}{1-a}+\frac{2}{a} y_{t-1}^{0} \frac{\varepsilon_{t}-1}{2}-\frac{2 z}{a}\left(1-\frac{\varepsilon_{t}-1}{2}\right)-\frac{z}{1-a} \\
& =c t+\varepsilon_{t}\left(-1+\frac{y_{t-1}^{0}}{a}+\frac{z}{a}\right) .
\end{aligned}
$$

The bubble equilibrium is not integrable and is a nonlinear function of $\underline{\varepsilon_{t}}$ by means of $y_{t}^{0}$. Nevertheless both $y_{t}^{F}$ and $y_{t}$ have a conditional variance given the past equal to $\sigma_{\varepsilon}^{2}$ and $\sigma_{\varepsilon}^{2}\left(1-\frac{y_{t-1}^{0}}{a}-\frac{z}{a}\right)^{2}$, respectively. We immediately note that $\left(1-\frac{y_{t-1}^{0}}{a}-\frac{z}{a}\right)^{2}$ cannot be larger than 1 for any admissible value $y_{t}^{0}$ and any negative $z$. Thus, when nonlinear stationary solutions are introduced, the forward solution is not the solution with minimum conditional variance [see a discussion of this criterion in Taylor (1977)]. The reason is the following one : the bubble component is based on the same innovation 
as the forward solution and can be negatively conditionally correlated with the forward solution.

\section{iii) Log-linearization of DSGE models}

It is common practice to log-linearize the equilibrium conditions derived from the DSGE models ${ }^{17}$ [see, e.g. Boragan et al. (2006) , Christiano et al. (2010) for examples]. The approach consists in replacing the (positive) variables by their log-expansion about a steady state $\mu$, say, that is, in approximating $y_{t}$ by:

$$
y_{t} \sim \mu+\mu\left(\log y_{t}-\log \mu\right)
$$

and next, replacing the initial model (2.1):

$$
y_{t}=a E_{t} y_{t+1}+z_{t}
$$

by the log-linearized model:

$$
\log y_{t}=a E_{t} \log y_{t+1}+z_{t} / \mu+(1-a)(\log \mu-1) .
$$

This log-linearized approximation is not innocuous in the search for stationary solutions of the DSGE model, even though the stationarity of $y_{t}$ is equivalent to the stationarity of $\log y_{t}$.

In the log-linearized version (3.17), the variable $\log y_{t}$ can be of any sign, resulting in an infinite number of stationary solutions, whereas in the initial model (2.1), in which the variable is positive, the number of solutions is still infinite, but smaller. This also implies that numerical procedures designed for finding "the" stationary solution to the log-linearized model (3.17) become questionable. The existing algorithms automatically select a unique solution and rely on numerical arguments rather than on either economic, or statistical arguments. 18

\footnotetext{
${ }^{17}$ or from dynamic stochastic partial equilibrium models.

${ }^{18}$ The same remarks apply to the log-linearized versions of the present value model [see e.g. Campbell, Shiller (1989)].
} 


\section{Identification of the Equilibrium and Im- pulse Response Analysis}

This section discusses the consequences of a possibly infinite set of stationary solutions of RE model (2.1). Given the multiplicity of solutions, the outcomes of numerical algorithms that select a unique solution need to be taken with caution. We address now the identification of the stationary equilibrium and the impulse response analysis.

At the beginning, we explain why the available observations can be used to statistically identify the stationary solution. Next, we point out that the dynamics of the stationary solution may be nonlinear, which requires a nonlinear approach to impulse response analysis.

Concerning the identification of the solution, our result differs from the recent literature [see e.g. Iskrev (2010), Komunjer, Ng (2011)]. The reason is that we prove the identification of a stationary solution from the initial model, whereas the literature usually considers the solution identification from the log-linearized vector autoregressive approximation of the initial model. Moreover, our approach is not a second-order identification, but instead a distribution-based identification. We use the assumption of i.i.d. errors in order to identify the nonlinear component of the dynamics.

\subsection{Identification}

In Section 3, we showed that the RE model (2.1) with $|a|<1$ has an infinite number of stationary solutions, which are derived from the forward solution by adding stationary submartingales of various types. These submartingales are Markov processes:

$$
y_{t}^{*} \equiv h\left(y_{t-1}^{*}, a\left(\varepsilon_{1 t}, \varepsilon_{2 t}\right)\right),
$$

where $h$ is a nonlinear transformation and $a\left(\varepsilon_{1 t}, \varepsilon_{2 t}\right)$ is a Gaussian noise derived from the innovations of exogenous variables that appear in the demand and supply. Under standard stability conditions such a process admits also a nonlinear infinite moving average representation :

$$
y_{t}^{*} \equiv H\left[\varepsilon_{1 t}, \varepsilon_{2 t}, \varepsilon_{1, t-1}, \varepsilon_{2, t-1}, \ldots\right],
$$

which can itself be written as a series expansion when $H$ satisfies appropriate 
differentiability conditions ${ }^{19}$. That is the Volterra expansion of the process written in terms of the i.i.d. sequence $\left(\epsilon_{t}\right)$ [see e.g. Priestley (1981), Volterra (2005)] :

$$
\begin{aligned}
y_{t}^{*}= & b+\sum_{h=0}^{\infty} a_{1 h}^{\prime} \varepsilon_{t-h}+\sum_{h=0}^{\infty} \sum_{k=0}^{\infty} a_{2 h k}^{\prime} \operatorname{vec}\left(\varepsilon_{t-h} \varepsilon_{t-k}^{\prime}\right) \\
+ & \sum_{\substack{h=0 \\
h \leq k}}^{\infty} \sum_{\substack{k=0 \\
h}}^{\infty} \sum_{l=0}^{\infty} a_{3 h k l}^{\prime} \operatorname{vec}\left[\operatorname{vec}\left(\varepsilon_{t-h} \varepsilon_{t-k}^{\prime}\right) \varepsilon_{t-l}^{\prime}\right]+\ldots,
\end{aligned}
$$

where $\varepsilon_{t}=\left(\varepsilon_{1 t}, \varepsilon_{2 t}\right)^{\prime}$ and vec $A$ denotes the vector obtained by stacking the columns of matrix $A$.

If both the forward solution and the additional submartingales have Volterra expansions, then the solution of the RE model also has a Volterra expansion.

The reason why multiple stationary rational expectation equilibria have been disregarded in the past literature was that the Volterra expansion is written only for linear stationary solutions and was therefore restricted to have zero moving average coefficients on terms of orders larger than 2 : $a_{2 h k}=0, a_{3 h k l}=0, \ldots$, Hence, all stationary solutions with nonlinear dynamics, such as speculative bubbles, volatility induced mean-reversion, and/or switching regimes have been artificially eliminated.

The joint model for $\left(y_{t}, z_{t}\right)$, where $y_{t}$ is the price and $z_{t}$ is an exogenous process (resp. dividend) also admits a Volterra expansion in i.i.d. errors $\left(\varepsilon_{1 t}, \varepsilon_{2 t}\right)$. Let us now discuss how to identify the stationary solution from an observed long trajectory of the joint process $\left(y_{t}, z_{t}\right)$, that is how to derive consistent approximations of the moving average coefficients $a_{1 h}, a_{2 h k}, a_{3 h k l}, \ldots$ and polynomials $\Phi$ and $\Theta$ that characterize the dynamics of $z_{t}$ (see equation

\footnotetext{
${ }^{19}$ These differentiability conditions are likely satisfied for the processes considered in Section 3.1.1-3.2.1. They do not hold for the basic exogenous innovations in the stationary martingale tree of Section 3.1.3. In this latter case, the additional submartingale may be of the type:

$$
y_{t+1}=\left(2 y_{t}+1\right) 1_{\epsilon_{1 t} \leq a_{1}}+\left(2 y_{t}-1\right) 1_{a_{1} \leq \epsilon_{1 t} \leq a_{2}}+1_{a_{2} \leq \epsilon_{1 t} \leq a_{3}}-1_{\epsilon_{1 t}>a_{3}},
$$

where $a_{j}, j=1,2,3$ are the quartiles of the distribution of $\epsilon_{1 t}$, assuming the impact of the exogenous innovation through $\varepsilon_{1 t}$, only. We get a moving average expansion which involves the indicator transformations of $\epsilon_{1 t}$.
} 
(2.3)). This identification problem is common in the time series literature on moving averages:

$$
y_{t}=A_{0} \epsilon_{t}+A_{1} \epsilon_{t-1}+A_{2} \epsilon_{t-2}+\cdots,
$$

that do not contain products of innovations. If the $\epsilon_{t}$ 's are i.i.d. and if $\epsilon_{1 t}$ is independent of $\epsilon_{2 t}$, we can identify matrix coefficients $A_{0}, A_{1}, \ldots$ up to a permutation of indexes 1,2 , of the $\epsilon$ 's and a multiplicative scale factor [see, e.g. Chan, Ho, Tong (2006)].

This identification result can be extended to nonlinear moving averages, i.e. to the Volterra expansion [see e.g. Christensen, Trott (1969), Koukoulas, Kalouptsidis (1995)]. Thus in such a RE model with multiple stationary equilibria, the RE equilibrium chosen by the agents can be identified from the joint observed paths of the price $y$ and exogenous variable $z$. That is, it is not necessary for identification to introduce any additional restriction leading to a unique stationary equilibrium.

The intuition underlying identification is that the econometrician and the economic agents have different information sets. More precisely, the econometrician observes ex-post $\left(y_{t}, z_{t}\right), t=1, \ldots, T$ over the whole period as well as the consequences of agents' decisions at all times, whereas at time $t$ the agents know only the past values of the variable up to time $t$. If $T$ is large, $T \rightarrow \infty$ and $\left(y_{t}\right)$ is strictly stationary, it is possible to estimate nonparametrically the nonlinear regression of $y_{t+1}$ on the past values $y_{t}, y_{t-1}, \ldots z_{t}, z_{t-1}, \ldots$ and next to estimate consistently parameter $a$ by regressing $y_{t}$ on $z_{t}$ and $\hat{y}_{t \mid t+1}$, where $\hat{y}_{t \mid t+1}$ denotes the predicted value. The difference with the standard instrumental variable approach, which implicitly selects the linear stationary solution, is the following : in the IV approach the expectation $E_{t} y_{t+1}$ is replaced by a linear prediction based on the instruments. Thus it differs from the true RE except for the linear stationary solution. Moreover this error in variable is not necessarily independent of the exogenous shocks. By considering nonparametric prediction, the error is asymptotically zero for any stationary solution. Of course this identification result says nothing about how the agents behaviours and expectations are coordinated to reach this identified collective rational equilibrium.

The identification result above and the associated semi-parametric estimation method could be difficult to apply in finite sample, especially to macroeconomic data. In the next subsection, we consider a parametric infinite set of stationary solutions constructed from the forward solution and a 
noncausal stable submartingale in order to illustrate the implementation of nonlinear impulse response analysis.

\subsection{Nonlinear IRF for a SVAR Model with Noncausal Component}

Let us assume $|a|<1$. The impulse response analysis describes how structural shocks propagate through the macroeconomy. The approach depends on the selected solution and differs from the standard linear analysis that is based on the forward solution only. The reason is that it has to take into account the nonlinear dynamics of the stationary submartingale. To illustrate this effect let us consider a solution with a noncausal stable submartingale :

$$
y_{t}(s, \rho)=y_{t}^{F}+y_{t}^{*}(s, \rho),
$$

and assume $z_{t}=\varepsilon_{1 t}-\varepsilon_{2 t}$, where $\varepsilon_{t}=\left(\varepsilon_{1 t}, \varepsilon_{2 t}\right)^{\prime} \sim N(0, I d)$. The forward solution is $y_{t}^{F}=z_{t}=\varepsilon_{1 t}-\varepsilon_{2 t}$.

Equation (3.5) implies that:

$$
\begin{aligned}
& y_{t}(s, \rho)=\frac{\varepsilon_{t}^{*}(s, \rho)}{1-\rho L^{-1}}+\varepsilon_{1 t}-\varepsilon_{2 t} \\
\Leftrightarrow \quad & (L-\rho) y_{t}(s, \rho)=L \varepsilon_{t}^{*}(s)+(L-\rho)\left(\varepsilon_{1 t}-\varepsilon_{2 t}\right) .
\end{aligned}
$$

The VARMA representation of $\left(y_{t}, z_{t}\right)$ written in terms of $\varepsilon_{t}^{*}(s), \varepsilon_{1 t}-\varepsilon_{2 t}$ :

$$
\left\{\begin{aligned}
(L-\rho) y_{t}(s, \rho)-(L-\rho) z_{t} & =L \varepsilon_{t}^{*}(s), \\
z_{t} & =\varepsilon_{1 t}-\varepsilon_{2 t},
\end{aligned}\right.
$$

is such that one root of the autoregressive polynomial lies inside the unit circle. In particular $\left(\varepsilon_{t}^{*}(s), \varepsilon_{1 t}-\varepsilon_{2 t}\right)$ is not the causal linear innovation of $\left(y_{t}(s, \rho), z_{t}\right)$.

The standard Box-Jenkins approach applied to the bivariate series $\left[y_{t}(s, \rho), z_{t}\right]$ is invalid for the following reasons : First, the Box-Jenkins method assumes the square integrability of $y_{t}(s, \rho)$, whereas this process has no mean. Second the Box-Jenkins approach is a linear approach, which cannot accommodate the nonlinear innovation $\left(\eta_{t}\right)$ in $(3.2)$.

The impulse response functions of a one-time shock at time $t$, represent the effects of shocks on $\left(\varepsilon_{1 t}-\varepsilon_{2 t}, \eta_{t}\right)^{\prime}$ (where $\left.\eta_{t}=a\left(\varepsilon_{1 t}, \varepsilon_{2 t}\right)\right)$, rather 
than on errors $\left[\varepsilon_{1 t}-\varepsilon_{2 t}, \varepsilon_{t}^{*}(s)\right]$, as $\varepsilon_{t}^{*}(s)$ is a nonfundamental error. This means that the impulse responses should not be based on the nonfundamental VARMA representation (4.2), but instead on the associated nonlinear causal autoregressive representation. Since bubbles are driven nonlinearly by the exogenous fundamentals, changes in these fundamentals can cause prices to overreact.

More precisely system (4.2) has to be rewritten in terms of the causal innovations as :

$$
\begin{aligned}
& \qquad \begin{aligned}
y_{t}(s, \rho) & =y_{t}^{F}+y_{t}^{*}(s, \rho), z_{t}=\varepsilon_{1 t}-\varepsilon_{2 t}, \\
\text { where }: y_{t}^{F} & =z_{t}=\varepsilon_{1 t}-\varepsilon_{2 t}, \\
y_{t}^{*}(s, \rho) & =g\left[y_{t-1}^{*}(s, \rho), a\left(\varepsilon_{1 t}, \varepsilon_{2, t}\right), s, \rho\right],
\end{aligned}
\end{aligned}
$$

and where $g$ and $a$ are given nonlinear functions.

The IRF are computed from stochastic shocks to structural errors $\varepsilon_{1 t}, \varepsilon_{2 t}$, by applying the nonlinear recursive scheme (4.3)-(4.5) [see Koop et al. (1996), Gourieroux, Jasiak (2005) for nonlinear IRF] and running nonlinear recursions. The shock can be either deterministic, or stochastic, and can possibly correspond to stressed situations [see Gourieroux, Jasiak (2015) for simulation of noncausal processes].

\section{Concluding Remarks}

A linear RE equilibrium model with current expectations always admits an infinite set of real-valued or nonnegative stationary dynamic equilibria even when $|a|<1$. These multiple intrinsic equilibria are derived from the forward solution and include stationary submartingales. These submartingales can feature speculative bubbles that explode and burst, volatility induced mean reversion and/or switching regimes. The existence of multiple stationary nonlinear equilibria requires an adequate analysis of identification, and a revised approach to impulse response analysis in RE models in order to account for the nonlinearities. The multiplicity of stationary equilibria challenges the current practices and principles of macroeconomic modeling, such as: 
-the assumption of a unique stationary equilibrium;

-the numerical algorithms that provide a specific solutions based on numerical criteria only;

-the idea that log-linearization is innocuous;

-the view that an explosive bubble will last indefinitely and dominate the fundamental solution;

- the idea that the forward solution has the minimal conditional variance; -the opinion that a RE model and its perfect-foresight analogue produce similar results;

-the estimation of parameters by standard instrumental variable approach, maximum likelihood, or Bayesian techniques. 
Moreover the possibility of stationary (non integrable) bubble component also challenges the main testing procedures of the present value model introduced in the literature. Let us consider these testing procedures.

i) The cointegration test [Diba, Grossman (1987)] is based on the idea that prices will be more nonstationary than the dividends, if there is a nonzero bubble component. This argument is no longer valid if the bubble is stationary.

ii) The variance bounds test introduced by Leroy, Porter (1981) [see also Cochrane (1992)] assumes the existence of the marginal variance of the price $y_{t}$. This assumption is not satisfied if the bubble component is stationary, then non integrable. We have also proved that a solution with bubble could be less volatile than the solution with zero bubble.

iii) The two step procedure introduced by West (1987) [see also Dezbakash, Demirgue-Kunt (1990)] is based on a standard instrumental variable estimation of the discount factor from the Euler restrictions. We mentioned in Section 3.3 that these standard estimation approaches are no longer valid. 


\section{Appendix 1}

\section{Equilibrium of Beliefs}

\section{i) Stochastic RE model}

For expository purpose, let us consider a stationary Markov process that satisfies the RE condition:

$$
y_{t}=a E_{t} y_{t+1} .
$$

Let us denote by $f\left(y_{t+1} \mid y_{t}\right)$ the transition pdf and $g\left(y_{t}\right)$ the marginal (stationary) pdf. The unknown transition pdf has to satisfy the following restrictions:

unitary mass restriction: $\int f\left(y_{+1} \mid y\right) d y_{+1}=1, \forall y$,

$\int y_{+1} f\left(y_{+1} \mid y\right) d y_{+1}=y / a, \forall y$.

This "number" of restrictions is much smaller than the number of possible transitions, which leaves room for an infinite number of solutions, i.e. of belief equilibria.

However, the stationarity assumption implies that the transition pdf has also to satisfy the Kolmogorov equation. There exists a function $g$ such that:

$$
\int f\left(y_{+1} \mid y\right) g(y) d y=g\left(y_{+1}\right), \forall y_{+1}
$$

where $\int g(y) d y=1$ and $g(y) \geq 0, \forall y$.

These inequality restrictions on function $g$ might considerably reduce the number of admissible stationary solutions. Note that the positivity restrictions concern the stationary pdf, not the process itself.

\section{ii) Deterministic perfect foresight model}

This outcome is very different from the outcome of its deterministic analogue: $y_{t}=a y_{t+1}$. In that case, the solutions are written in terms of process $\left(y_{t}\right)$. These are equilibria of trajectories, and no longer equilibria of beliefs. These solutions are given by $y_{t}=y_{0} / a^{t}, t \geq 0$, where $y_{0}$ is a (possibly stochastic) initial value. If $0<a<1$, we get the unique stationary solution with the choice of $y_{0}=0$, that is $y_{t}=0, \forall t$. 


\section{Appendix 2}

\section{Explosive Bubble Models in the Literature}

Explosive bubbles are dynamic patterns that represent a short (stochastic) explosion followed by a crash. Several examples of (sub)martingales with explosive bubbles were considered in the economic literature or RE models.

We discuss below the most commonly used models of bubble dynamics that appeared in the literature. These models usually present martingale processes, corresponding to the case $a=1, z_{t}=0$.

i) Blanchard (1979).

The bubble dynamics is defined by:

$$
Y_{t+1}=\left\{\begin{array}{cl}
\frac{1}{\pi} Y_{t}, & \text { with probability } \pi, \\
0 & \text { with probability } 1-\pi .
\end{array}\right.
$$

This process represents a single bubble, with 0 as an absorbing state. The rate of explosion of the bubble is fixed and equal to $1 / \pi$. It is strictly larger than the average rate of explosion, which is equal to 1 by the martingale condition.

The regime indicator appearing in the bubble model above may be defined as a function of the standard Gaussian nonlinear innovation $\left(\epsilon_{t}\right)$ of the exogenous process (see Gouriéroux, Laffont and Monfort (1982), p.42), that leads to the model:

$$
Y_{t+1}=\frac{1}{\pi} Y_{t} \mathbb{1}_{\epsilon_{t+1}<\Phi^{-1}(\pi)},
$$

where $\Phi$ is the c.d.f. of the standard normal.

In an alternative specification, the indicator variable may alse be assumed as independent of the innovation process. Hence, two different choices of the information set are available.

The bubble has been considered as a martingale component to be added to the forward solution in order to obtain an additional solution of RE model (2.1). However, the martingales in the above model are nonstationary and asymptotically tend to zero. Therefore they induce no long term effects of self-fulfilling prophecies.

ii) Blanchard and Watson (1982). 
This bubble model eliminates zero as an absorbing state. The dynamics of the bubble is given by:

$$
Y_{t+1}= \begin{cases}\frac{1}{\pi} Y_{t}+\epsilon_{t+1}, & \text { with probability } \pi, \\ \epsilon_{t+1}, & \text { with probability } 1-\pi,\end{cases}
$$

where, for instance, $\left(\epsilon_{t}\right)$ is an i.i.d. zero mean sequence constructed from the nonlinear Gaussian innovation of the exogenous process. This process takes positive as well as negative values. As before, the regime indicator can be a function of $\left(\epsilon_{t}\right)$, or it can be independent of the innovation process. This model allows for erratic changes in $Y_{t}$ during the explosion spell. It also allows for multiple explosive bubbles. When $\varepsilon_{t} \sim N\left(0, \sigma^{2}\right)$ this process is a limiting case of the switching process analyzed in Section 3.1.4, corresponding to $a=1$.

\section{iii) Evans (1991).}

Evans proposed an explosive bubble with a stochastic rate of explosion:

$$
Y_{t+1}= \begin{cases}u_{t+1} Y_{t}, & \text { if } Y_{t}<\alpha \\ \left(\delta+\frac{1}{\pi} \theta_{t+1}\left(Y_{t}-\delta\right)\right) u_{t+1}, & \text { if } Y_{t}>\alpha\end{cases}
$$

where $\alpha, \delta$ are parameters such that $0<\delta<\alpha,\left(u_{t}\right)$ is an i.i.d. process with $u_{t} \geq 0, E_{t}\left(u_{t+1}\right)=1$ for all $t,\left(\theta_{t}\right)$ is an i.i.d. Bernoulli process $\mathcal{B}(1, \pi)$. As long as $Y_{t}<\alpha$, the bubble erupts at a faster mean rate $1 / \pi$, but bursts with probability $1-\pi$. The model requires an information set strictly larger than the set generated by the exogenous innovation process.

\section{iv) Kamihigashi (2011)}

The dynamics is defined by :

$$
\log Y_{t+1}=\mu\left(Y_{t}\right)+\varepsilon_{t+1} / c\left(Y_{t}\right)
$$

where the $\varepsilon_{t}^{\prime} s$ are i.i.d. with a symmetric distribution. Then it is proved (see Corollary 5.1 in Kamihigaski (2011) that the process is recurrent if

- function $c$ is decreasing from $(0, \infty)$ to $(0, \infty)$, with $0<c(\infty) \leq c(0)<$ $\infty$,

and

- $\mu(\infty)<0<\mu(0)$. 
The recurrence property ensures that the process will pass an infinite number of times in any neighbourhood of any positive real value. In other words the process will neither explode with probability 1, nor asymptotically tend to 0 with probability 1 . The recurrence property does not imply the strong stationarity of the process. ${ }^{20}$ For instance the random walk without drift corresponds to the limiting case : $c(y)=c$ constant, $\mu(y)=0$, is recurrent and nonstationary. To get the stationarity additional conditions have to be introduced. More precisely it is known that any recurrent Markov process has a stationary distribution with density $f(y)$, say, which corresponds to a positive measure. Thus $f(y)>0$, but does not necessarily sum up to one. This limiting density is the solution of the Kolmogorov's equation :

$$
f(y)=\int_{0}^{\infty} \frac{c(z)}{y} g\{c(z)[\log y-\mu(z)]\} f(z) d z,
$$

where $g$ denotes the density of the noise $\varepsilon$.

Thus the strong stationarity of the process requires the following necessary condition :

$$
\int_{0}^{\infty} f(y) d y=1 \text {, i.e. the unit mass restriction, }
$$

plus additional stability conditions.

From the current discrete time literature the conditions on $p$ and $c$ to get the strong stationarity are not yet known.

\section{Appendix 3}

\section{Necessity of Transversality Conditions}

Before analyzing the potential role of transversality conditions in our framework, let us first remark that

i) the main part of the literature on transversality conditions focuses on deterministic optimizations [see e.g. Michel (1990), Ekeland, Scheinkman (1986), Kamihigashi (2001), (2008)], and cannot be immediately extended to optimization in a stochastic environment.

\footnotetext{
${ }^{20}$ See Gourieroux, Jasiak (2016) for the test of the martingale hypothesis under the recurrence assumption.
} 
ii) Moreover, as mentioned in Kamihigashi (2001), "the Euler equation is derived from the optimality requirement that no gain be achieved by choosing a path that deviates from the optimal path, but eventually returns to it. By contrast the transversality condition is derived from the requirement that no gain be achieved by choosing a path that deviates from it forever". In our case the stationary bubble components create paths that will not deviate forever.

Let us now consider a standard Consumption based Capital Asset Pricing Model (CCAPM) to show that the transversality conditions do not eliminate the stationary bubbles. The intertemporal optimization problem is :

$$
\max _{\left(\alpha_{t}, C_{t}\right)} \sum_{t=0}^{\infty} \beta^{t} E_{0} U\left(C_{t}\right) \text {, s.t. } C_{t}+\alpha_{t} p_{t}=R_{t}+\alpha_{t-1} p_{t}, \forall t,
$$

$C_{t}$ is the consumption at date $t$ with the price of the consumption good fixed to 1 for expository purpose, $\alpha_{t}$ the quantity of risky asset, $p_{t}$ its unitary price $\left(p_{t}>0\right), R_{t}, R_{t}>0$ and exogenous income, $U$ the utility function and $\beta$ a discount, $0<\beta<1$.

After eliminitating the current and future budget constraints, we get the intertemporal optimization :

$$
\max _{\left(\alpha_{t}\right)} \sum_{t=0}^{\infty} \beta^{t} E_{0} U\left[R_{t}+p_{t}\left(\alpha_{t-1}-\alpha_{t}\right)\right] .
$$

The optimum $\left(\alpha_{t}^{*}\right)$ (if it exists) satisfies the Euler restrictions :

$$
p_{t} U^{\prime}\left[R_{t}+p_{t}\left(\alpha_{t-1}^{*}-\alpha_{t}^{*}\right)\right]=\beta E_{t}\left[p_{t+1} U^{\prime}\left[R_{t+1}+p_{t+1}\left(\alpha_{t}^{*}-\alpha_{t+1}^{*}\right)\right]\right\}, \forall t,
$$

and also the inequalities:

$$
C_{t}^{*}=R_{t}+p_{t}\left(\alpha_{t-1}^{*}-\alpha_{t}^{*}\right)>0, \forall t .
$$

Let us now assume the equilibrium on the financial market :

$$
\alpha_{t}^{*}=\alpha_{0 t}, \forall t,
$$

where the asset supply $\alpha_{0 t}$ is exogenous and assumed to decrease :

$$
\alpha_{0, t-1}>\alpha_{0, t}(>0) \text {. }
$$


This condition implies that the sequence $\alpha_{0, t}$ will tend to a positive limit when $t$ tends to infinity, and also that $\alpha_{t-1}^{*}-\alpha_{t}^{*}=\alpha_{0, t-1}-\alpha_{0, t}$ will tend to zero.

Under assumption (a.6) the inequalities (a.5) are automatically satisfied.

Let us now consider a stationary positive submartingale $\left(y_{t}\right)$ satisfying :

$$
y_{t}=\beta E_{t} y_{t+1} .
$$

Lemma : Let us consider a CRRA utility function :

$U(C)=C^{\gamma} / \gamma$, with $0<\gamma<1$. There exists (at least) a positive process $\left(p_{t}\right)$ solution of :

$$
p_{t} U^{\prime}\left[R_{t}+p_{t}\left(\alpha_{0, t-1}-\alpha_{0, t}\right)\right]=y_{t}, \forall t
$$

Proof :

Indeed the function of $p_{t}$ in the left hand side is equal to :

$$
G\left(p_{t}\right)=\gamma p_{t} /\left[R_{t}+p_{t}\left(\alpha_{0, t-1}-\alpha_{0, t}\right)\right]^{1-\gamma} .
$$

This function is such that : $G(0)=0, G(\infty)=\infty$. This provides the result.

\section{QED}

When $t$ tends to infinity, this solution is equivalent to :

$$
p_{t} \sim \frac{1}{\gamma} R_{t}^{1-\gamma} y_{t}
$$

Is this solution compatible with the intertemporal optimization problem, in particular with the "transversality conditions"? The answer is yes. Indeed, the utility function $U$ is unbounded, and we can apply Proposition 4.3 in Kamihigashi (2005) :

if $\sum_{t=1}^{\infty} \beta^{t} E_{0} U\left[R_{t}+p_{t}\left(\alpha_{0, t-1}-\alpha_{0, t}\right)\right]<\infty$, the transversality conditions are automatically satisfied. Otherwise, the lifetime utility is infinite at the optimum and the transversality conditions are not necessary. 
As noted in Kamihigashi (2005), p 1323: "This case is usually ruled out in practice and (the conditional finite lifetime utility) assumed implicitly or taken for granted in applied studies". This practice can eliminate important solutions.

How to explain this larger number of solutions ? Of course the limiting case : $\alpha_{0, t}=\alpha_{0}$ independent of time, would not be compatible with the equilibrium on financial market. Indeed the investor would have always the same quantity of asset, and no need to trade for updating his/her portfolio. In our example, there are trades at any finite dates, since the number of shares in the portfolio diminishes (but not necessarily the value of the portfolio), and the floating will tend to zero. From an economic viewpoint the multiplicity of solutions is likely due to the fact that the virtual financial market at $t=\infty$ cannot exist.

From a mathematical viewpoint, the multiplicity of stationary solutions satisfying the positivity and the standard conditions for the optimization is likely due to the fact that the value of the objective function at the optimum is infinite. Indeed the stationary bubbles are not integrable. In such a framework, it is not possible to select among these solutions on the basis of the value of the intertemporal expected utility only. This problem has already been encountered in a deterministic framework for the Ramsey's model of economic growth. In this framework the idea is to choose between the solutions by considering the speed at which the intertemporal utilities up to a finite time $T$, say, tend to infinity, when $T$ tends to infinity. In other words the objective function has to be modified. Such a change of criterion appears for instance in the notion of sustainable development [see e.g. Chichilnisky (1996), Asheim, Ekeland (2016)]. In the stochastic framework, the deterministic time $T$ would have to be replaced by a sequence $\tau_{T}$ of (stochastic) stopping times tending to $\infty$ with $T$. Such an analysis and the possible economic interpretations of such new criteria are beyond the scope of the present paper. 


\section{References}

[1] Anderson, G. (2008): "Solving Linear Rational Expectations Models: A Horse Race", Computational Economics, 31, 95-113.

[2] Asheim, G., and I., Ekeland (2016) : "Resource Conservation Across Generations in a Ramsey-Chichilnisky Model", forthcoming Economic Theory.

[3] Bec, F. Ben Salem, M., and M., Carrasco (2010) : "Detecting Mean Reversion in Real Exchange Rates from a Multiple Regime STAR Model", Annals of Economics and Statistics, 99/100, 395-422.

[4] Bertocchi, G. (1991): "Bubbles and Inefficiencies", Economic Letters, 35, 117-122.

[5] Black, F. (1974) : "Uniqueness of the Price Level in Monetary Growth Models with Rational Expectations", Journal of Economic Theory, 7, 5365 .

[6] Blanchard, O. (1978) : "Backward and Forward Solutions for Economics with Rational Expectations", DP 627, Harvard University.

[7] Blanchard, O. (1979) : "Speculative Bubbles, Crashes and Rational Expectations", Economic Letters, 3, 387-389.

[8] Blanchard, O., and S. Fisher (1989): "Lectures on Macroeconomics", MIT Press.

[9] Blanchard, O., and C. Kahn (1980): "The Solution of Linear Difference Models Under Rational Expectations", Econometrica, 48, 1305-1312.

[10] Blanchard, O. J. and M. W. Watson (1982): Bubbles, Rational Expectations and Financial Markets. In Crises in the Economic and Financial Structure, ed. by P. Wachtel, 295-316. Lexington, MA: D.C. Heathand Company.

[11] Blanchard, O. and P. Weil (2001): "Dynamic Efficiency, the Riskless Rate and Debt Ponzi Games Under Uncertainty", in Advances in Macroeconomics, Vol. 1, Berkeley Electronic Press. 
[12] Boragan, A., Fernandez-Villaverde, J., and J. Rubio-Ramirez (2006): "Comparing Solution Methods for Dynamic Equilibrium Economies", Journal of Economic Dynamics and Control, 30, 2477-2508.

[13] Borkovec, M. and C. Kluppelberg (2001): "The Tail of the Stationary Distribution of an Autoregressive Process with ARCH(1) Errors", Annals of Applied Probability, 11, 1220-1241.

[14] Brock, W. (1975): "A Simple Perfect Foresight Monetary Model", Journal of Monetary Economics, 1, 133-150.

[15] Broze, L., Gourieroux, C., and A., Szafarz (1995) : "Solutions of Multivariate Rational Expectations Models", Econometric Theory, 11, 229-257.

[16] Brunnermeier, M. (2008) : "Bubbles", in S., Durlauf and L. Blume, eds, New Palgrave Dictionary of Economics, 2nd edition, Palgrave MacMillan, London.

[17] Caballero, R. (2010) : "Macroeconomics After the Crisis : Time to Deal with the Pretense of Knowledge Syndrome", Journal of Economic Perspective, 24, 85-102.

[18] Camerer, C. (1989): "Bubbles and Fads in Asset Prices", Journal of Economic Surveys, 3, 3-45.

[19] Campbell, J., and R., Shiller (1989) : "The Dividend-Price Ratio and Expectations of Future Dividends and Discount Factors", Review of Financial Studies, 1, 195-228.

[20] Chan, K., Ho, L. and H. Tong (2006): "A Note on Time Reversibility of Multivariate Linear Processes", Biometrika, 93, 221-227.

[21] Chichilnisky, G. (1996) : "An Axiomatic Approach to Sustainable Development", Soc. Choice Welfare, 13, 231-257.

[22] Christiano, L., Trabandt, M. and K. Walentin (2010): "DSGE Models for Monetary Policy Analysis", NBER 16074.

[23] Christensen, G., and G., Trott (1969) : "On the Uniqueness of the Volterra Series", IEEE Trans. Aut. Control, 14, 759-760. 
[24] Cochrane, J. (1992) : "Explaining the Variance of Price Dividend Ratios", Review of Financial Studies, 5, 243-280.

[25] Conley, T., Hansen, L., Luttmer, E., and J., Scheinkman (1997) : "Short Term Interest Rates as Subordinated Diffusions", Review of Financial Studies, 10, 525-577.

[26] Cox, J., Ross, S. and M. Rubinstein (1979): "Option Pricing: A Simplified Approach", Journal of Financial Economics, 7, 229-263.

[27] Dezbakhash, H., and A., Demirgue-Kunt (1990) : "On the Presence of Speculative Bubbles in Stock Prices", The Journal of Financial and Quantitative Analysis, 25, 101-112.

[28] Diba, B., and H., Grossman (1987) : "On the Inception of Rational Bubbles", Quarterly Journal of Economics, 87, 697-700.

[29] Diba, B., and H., Grossman (1988)a: "Explosive Rational Bubbles in Stock Prices ?", The American Economic Review, 78, 520-530.

[30] Diba, B. and H. Grossman (1988)b "The Theory of Rational Bubbles in Stock Prices", The Economic Journal, 98, 746-754

[31] Dufour, J.M., Khalaf, L., and M., Kichian (2006) : "Inflation Dynamics and the New Keynesian Phillips Curve : An Identification Robust Econometric Analysis", Journal of Economic Dynamics and Control, 30, 1707-1727.

[32] Dufour, J.M., Khalaf, L., and M., Kichrian (2010) : "IdentificationRobust Analysis of DSGE and Structural Macroeconomic Models", Journal of Monetary Economics, 60, 340-350.

[33] Durett, R. (1996) : "Stochastic Calculus : A Practical Introduction", Boca Raton, CRC Press.

[34] Ekeland, I., and J., Scheinkman (1986) : "Transversality Conditions for Some Infinite Horizon Discrete Time Optimization Problems", Mathematics of Operations Research, 11, 216-219.

[35] Enders, Z., and H., Hakenes (2014) : "On the Existence and Prevention of Speculative Bubbles", Univ. of Heidelberg, DP 567. 
[36] Evans, G. (1991): "Pitfalls in Testing for Explosive Bubbles in Asset Prices", The American Economic Review, 81, 922-930.

[37] Evans, G. and S. Honkapohia (1986): " A Complete Characterization of ARMA Solutions to Linear Rational Expectation Models", Review of Economic Studies, 53, 227-239.

[38] Flood, R., and P., Garber (1980): "Market Fundamentals Versus Price Level Bubbles. The First Tests", Journal of Political Economy, 88, 745770 .

[39] Flood, R., and R., Hodrick (1990) : "On Testing for Speculative Bubbles", Journal of Economic Perspectives, 4, 85-101.

[40] Froot, K., and M., Obstfeld (1991) : "Intrinsic Bubbles : The Case of Stock Prices", American Economic Review, 81, 1189-1214.

[41] Fuhrer, J., and G., Oliver (2004) : "Estimating Forward Looking Euler Equations with GMM Estimators : An Optimal Instruments Approach", Technical Report, Federal Reserve Bank of Chicago.

[42] Gali, J., Gerthler, M., and J., Lopez-Salido (2001) : "European Inflation Dynamics", European Economic Review, 45, 1327-1270.

[43] Gourieroux, C., and J., Jasiak (2005) : "Nonlinear Impulse Response Functions", Annales d'Economie et de Statistique, 78, 1-33.

[44] Gourieroux, C., and J., Jasiak (2015) : "Filtering, Prediction and Simulation Methods for Noncausal Processes", Journal of Time Series Analysis, 37, 405-430.

[45] Gourieroux, C., and J., Jasiak (2016) : "Robust Analysis of the Martingale Hypothesis", CREST DP.

[46] Gourieroux, C., Laffont, J.J., and A., Monfort (1982) : "Rational Expectation in Dynamic Linear Models : Analysis of the Solutions", Econometrica, 50, 409-425.

[47] Gourieroux, C., and J.M., Zakoian (2016) : "Explosive Bubble Modelling by Noncausal Process", forthcoming Journal of the Royal Statistical Society. 
[48] Gurkaynak, R. (2008) : "Econometric Tests of Asset Price Bubbles : Taking Stock", Journal of Economic Surveys, 22, 166-186.

[49] Hall, R. (1978): "Stochastic Implications of the Life Cycle Permanent Income Hypothesis: Theory and Evidence", Journal of Political Economy, 86, 971-988.

[50] Harrison, M., and D., Kreps (1979) : "Martingales and Arbitrage in Multiperiod Securities Markets", Journal of Economic Theory, 20,0381408.

[51] Hong, H., and D., Sraer (2013) : "Quiet Bubbles", Journal of Financial Economics, 110, 596-606.

[52] Iskrev, N. (2010): "Local Identification in DSGE Models", Journal of Monetary Economics, 57, 189-202.

[53] Kamihigashi, T. (1998): "Uniqueness of Asset Prices in an Exchange Economy with Unbounded Utility", Economic Theory, 12, 103-122.

[54] Kamihigashi, T. (2001) : "Necessity of Transversality Conditions for Infinite Horizon Problems", Econometrica, 69, 995-1012.

[55] Kamihigashi, T. (2004) : "Necessity of the Transversality Condition for Stochastic Models with Bounded or CRRA Utility", Journal of Economic Dynamics and Control, 29, 1313-1329.

[56] Kamihigashi, T. (2008): "Transversality Conditions and Dynamic Economic Behavior", in New Palgrave Dictionary of Economics, 2nd edition, ed. Durlauf, S. and Blume, L.

[57] Kamihigashi, T. (2011) : "Recurrent Bubbles", Japanese Economic Review, 62, 27-62.

[58] Klein, P. (2000): "Using the Generalized Schur Form to Solve a Multivariate Linear Rational Expectations Model", Journal of Economic Dynamics and Control, 24, 1405-1423.

[59] Komunjer, I., and S. Ng, (2011): "Dynamic Identification of DSGE Models", 79, 1995-2032. 
[60] Koop, G., Pesaran, M., and S., Potter (1996) : "Impulse Response Analysis in Nonlinear Multivariate Models", Journal of Econometrics, 74, 119-147.

[61] Koukoulas, P., and N., Kalouptsidis (1995) : "Nonlinear System Identification Using Gaussian Inputs", IEEE Trans. Signal Process., 43, 18311841.

[62] Kutoyants, Y. (2004) : "Statistical Inference for Ergodic Processes", Springer.

[63] Leeper, E., Walker, T., and S., Yang (2013) : "Fiscal Foresight and Information Flows", Econometrica, 81, 1115-1145.

[64] LeRoy, S., and R., Porter (1981) : "The Present-Value Relation : Tests Based on Implied Variance Bounds", Econometrica, 49, 555-574.

[65] Ling, S. (2007): "A Double AR(1) Model: Structure and Estimation", Stat. Sin., 17, 161-175.

[66] Ljungqvist, L. and T. Sargent (2000): "Recursive Macroeconomic Theory", Cambridge MIT Press.

[67] Lucas, R. (1978): "Asset Prices in an Exchange Economy", Econometrica, 46, 1429-1445.

[68] McCallum, B. (1983) : "On Non-Uniqueness in Rational Expectations Models : An Attempt at Perspective", Journal of Monetary Economics, $11,139-168$.

[69] Miao, J. (2004) : "Introduction to Economic Theory of Bubble", Journal of Mathematical Economics, 53, 130-136.

[70] Michel, P. (1990) : "Some Clarifications on the Transervasilty Condition", Econometrica, 58, 705-723.

[71] Montrucchio, L. and F. Privileggi (2001): "On Fragility of Bubbles in Equilibrium Asset Pricing Models of Lucas-Type", Journal of Economic Theory, 101, 158-188. 
[72] Nielsen, H. and A. Rahbek (2014): "Unit Root Vector Autoregression with Volatility Induced Stationarity", Journal of Empirical Finance, 29, 144-167.

[73] Obstfeld, M. and K. Rogoff (1983): "Speculative Hyperinflations in Maximizing Models: Can We Rule Them Out", Journal of Political Economy, 91, 675-687.

[74] Pesaran, H., and R., Smith (2011) : "Beyond the DSGE Straitjacket", DP 1138, Cambridge University.

[75] Priestley, M. (1981) : "Spectral Analysis and Time Series", Academic Press, San Diego.

[76] Rosenblatt, M. (2000) : "Gaussian and Non Caussian Linear Time Series and Random Fields", Springer Verlag.

[77] Samuelson, P. (1985): "An Exact Consumption Loan Model of Interest with or without the Social Contrivance of Money", Journal of Political Economy, 66, 467-482.

[78] Sargent, T., and N. Wallace (1975): "Rational Expectations, the Optimal Monetary Instrument and the Optimal Money Supply Rule", Journal of Political Economy, 83, 241-254.

[79] Schorfheide, F. (2011) :"Estimation and Evaluation of DSGE Models : Progress and Challenges", DP Federal Reserve Bank of Philadelphia.

[80] Shiller, R. (1978): "Rational Expectation and the Dynamic Structure of Macroeconomic Models: A Critical Review", Journal of Monetary Economics, 4, 1-49.

[81] Sims, C. (2002): "Solving Linear Rational Expectations Models", Computational Economics, 20, 1-20.

[82] Taylor, J. (1977) : "Condition for Unique Equilibrium in Stochastic Macroeconomic Models with Rational Expectation", Econometrica, 45, $1372-1385$.

[83] Tirole, J. (1985): "Asset Bubbles and Overlapping Generations", Econometrica, 53, 1499-1528. 
[84] Tong, H (1990) : "Nonlinear Time Series", Oxford Univ. Press.

[85] Velinov, A., and W., Chen (2013) : "Are There Bubbles in Stock Prices? Testing for Fundamental Shocks", DP 1375, DIW, Berlin.

[86] Volterra, V. (2005) : "Theory of Functionals and of Integrand and Integrodifferential Equations", Dover, New-York.

[87] West, K. (1987) : "A Specification Test for Speculative Bubbles", The Quarterly Journal of Economics, 102, 553-580.

[88] Williams, D. (1991): "Probability with Martingale", Cambridge University Press. 
Noncausal Cauchy AR(1), coeff $=0.5$

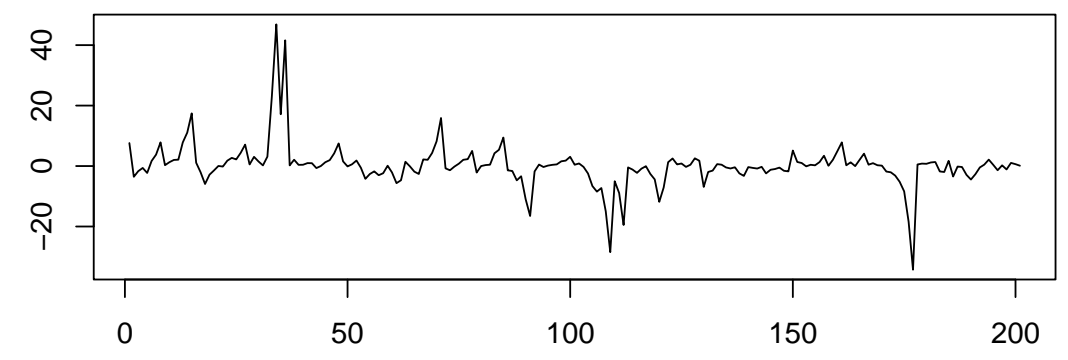

Noncausal Cauchy AR(1), coeff $=0.8$

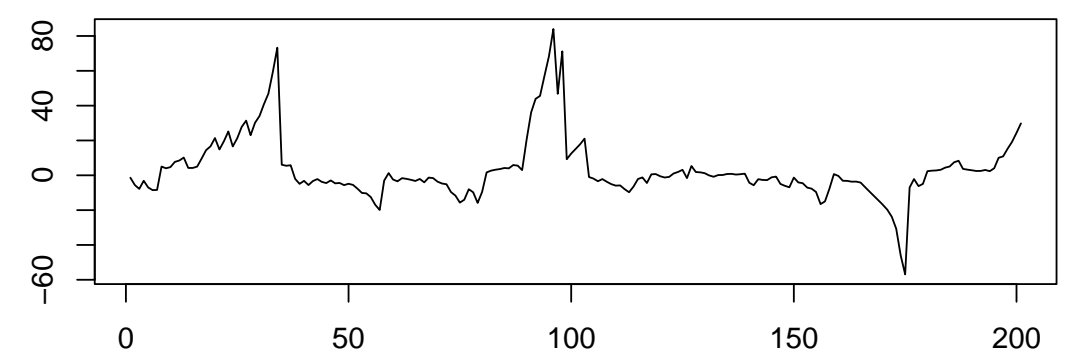

Figure 1: Path of a Noncausal Stable Process 


\section{Diffusion a)}

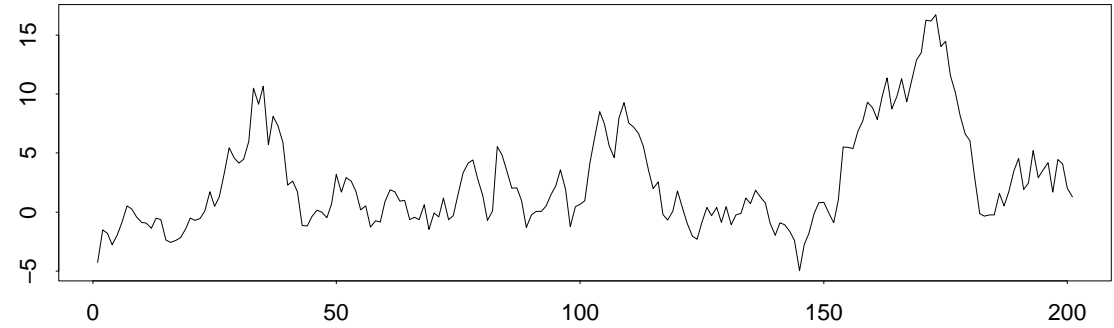

Diffusion b)

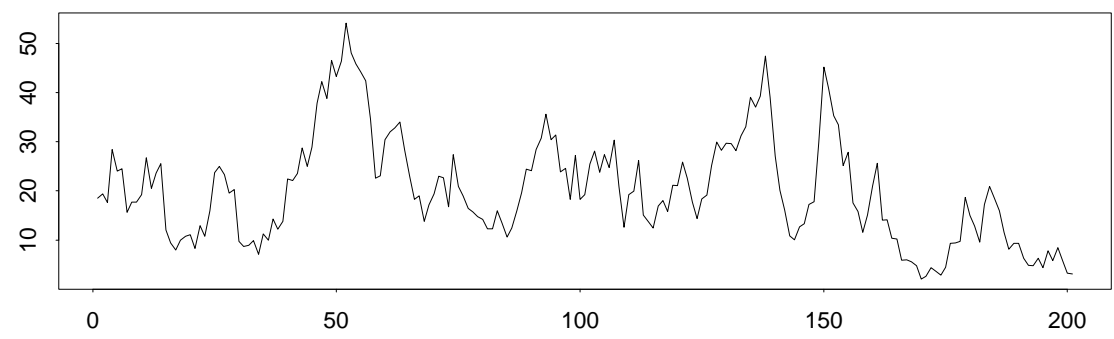

Figure 2: Path of a Process with Volatility Induced Mean-Reversion 


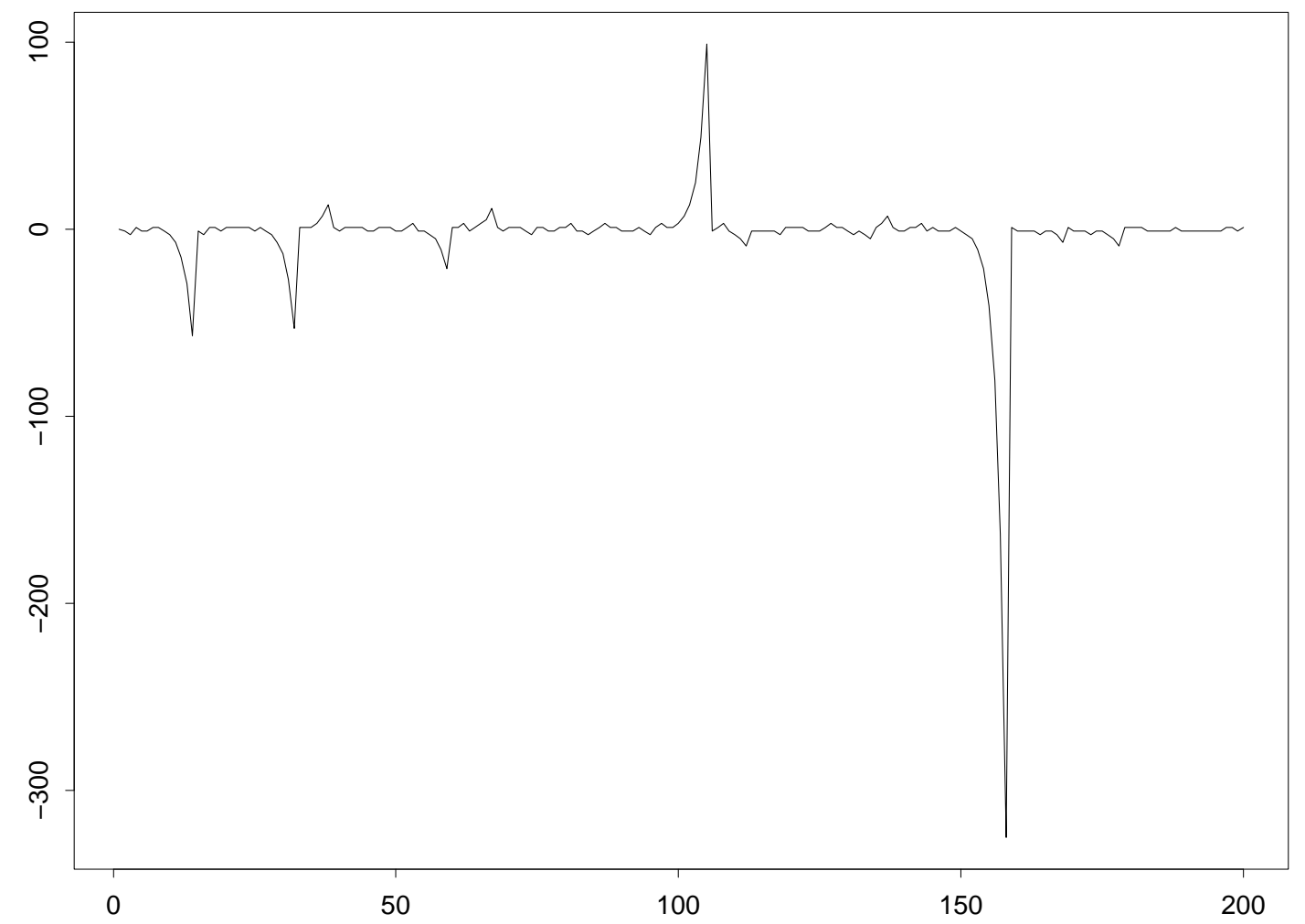

Figure 3: Path of the Stationary Martingale Tree 